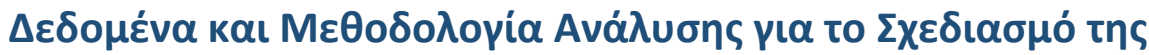

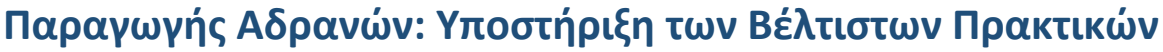

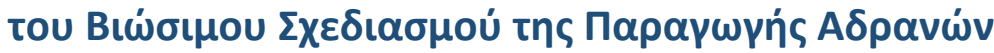

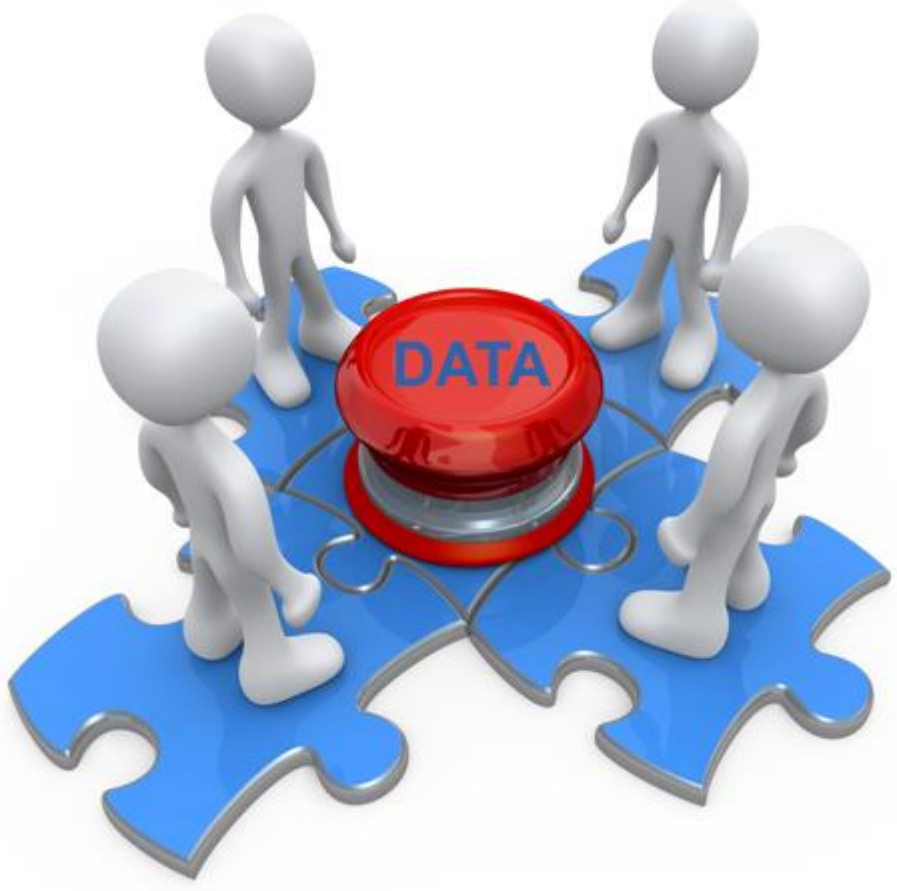

Октம்ßрเо૬ 2014

Technical University of Crete

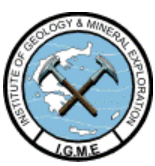

Provincia autonoma di Trento

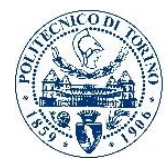

POLITECNICO DI TORINO 



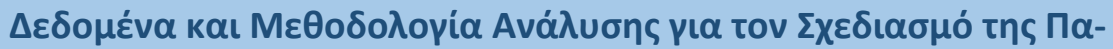

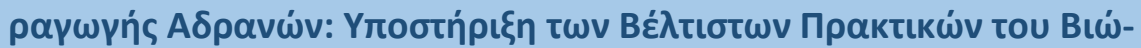

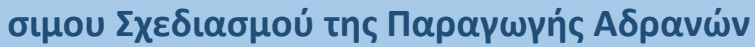

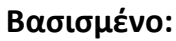

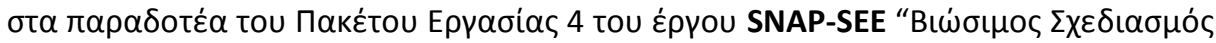

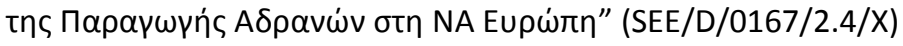

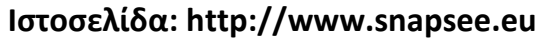

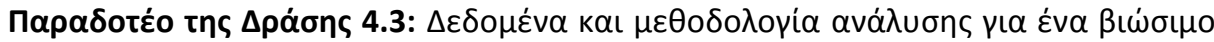

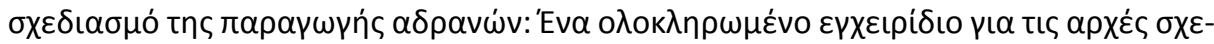

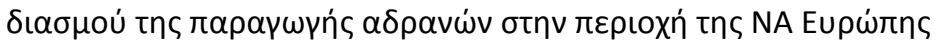

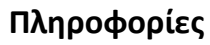

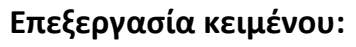

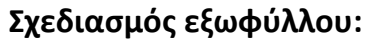

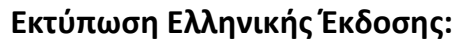

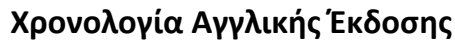

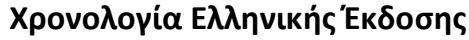

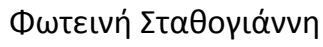

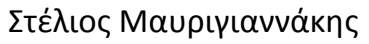

I.Г.M.E.M

Málos 2014

Окти́ßріоธ 2014

\section{Aтотоínon EuӨúvns}

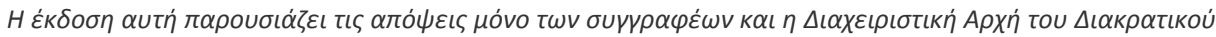

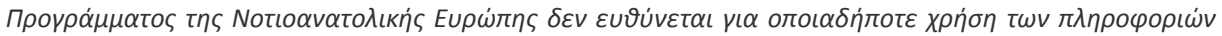
autẃv. CSNAP-SEE project 2014 


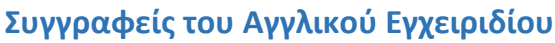

\begin{tabular}{|c|c|}
\hline & 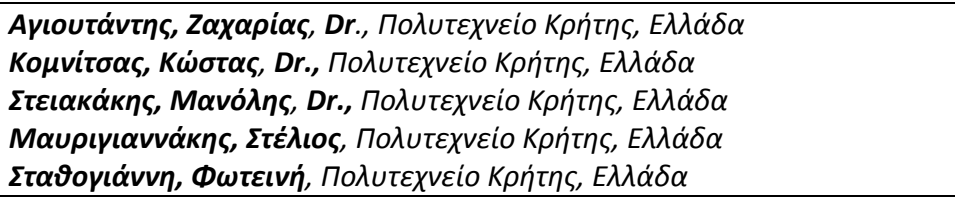 \\
\hline & 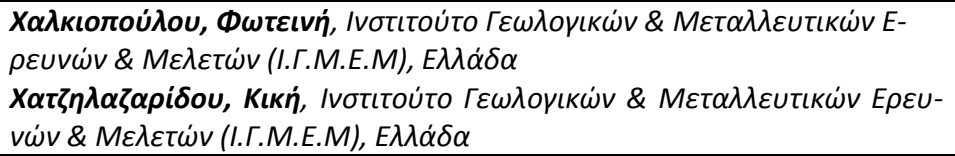 \\
\hline Provincia autonoma di Trento & 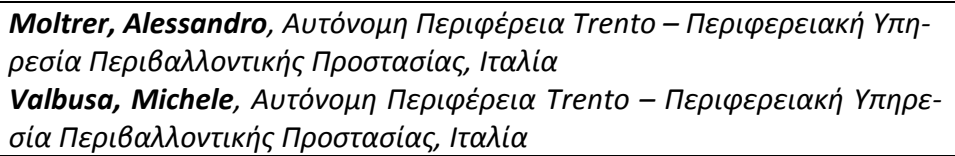 \\
\hline & 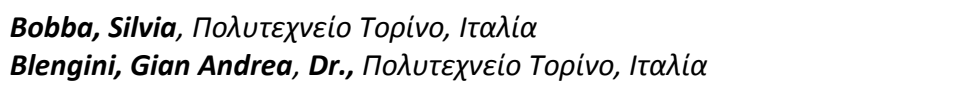 \\
\hline
\end{tabular}

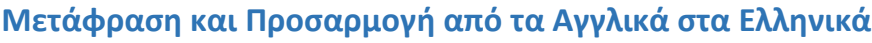

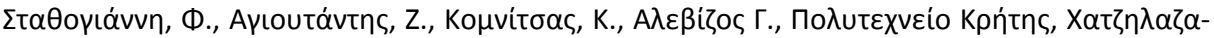

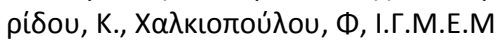

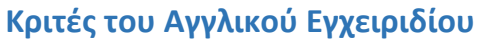

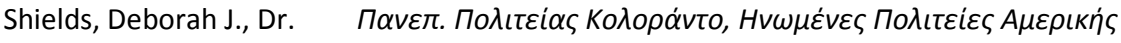 \\ O’Brien, Jim

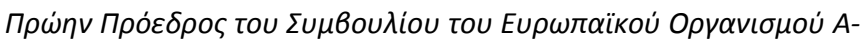

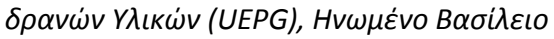 \\ Simic, Vladimir, Dr.

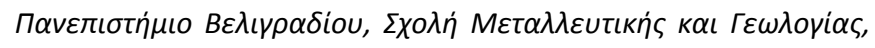 $\Sigma \varepsilon \rho B i \alpha$}

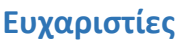

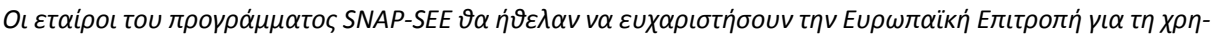

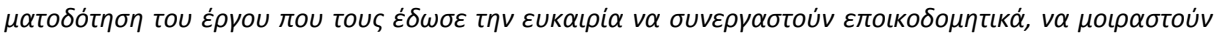

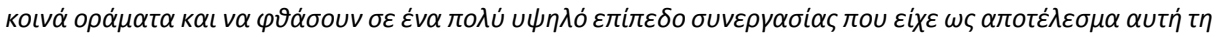

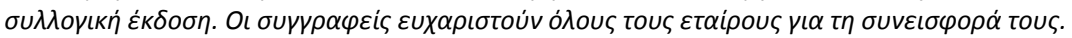

\section{Digital Object Identifier}

doi: 10.5474/snap-see-WP4-EL

\section{Eтعроаvaфора́}

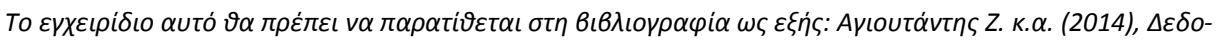

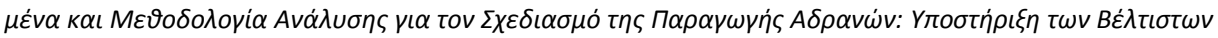

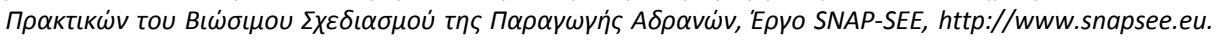
doi: 10.5474/snap-see-WP4-EL 


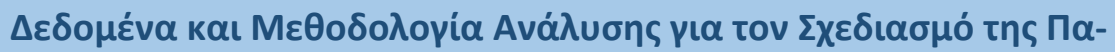

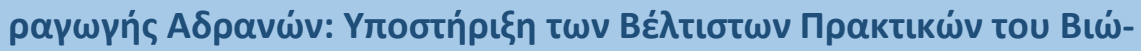

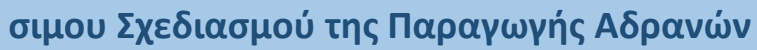

TEPIEXOMENA

Проo'ín。

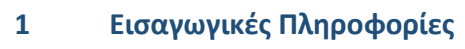

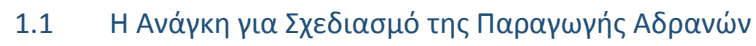

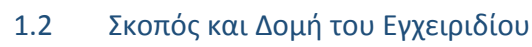

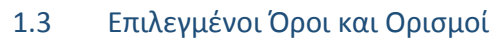

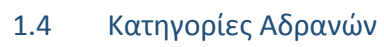

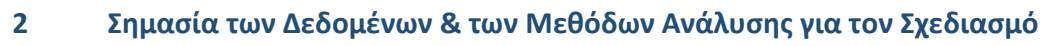

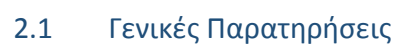

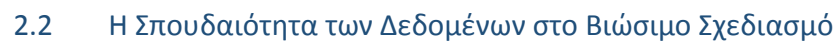

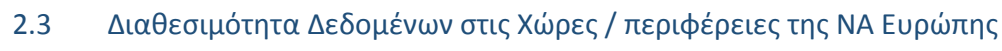

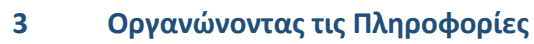

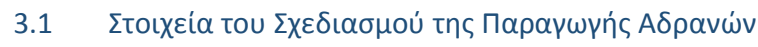

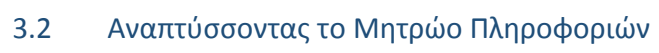

3.3 Пврьорเбноі

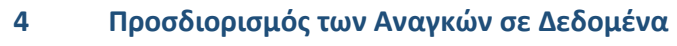

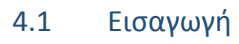

4.2 Mopфń $\tau \omega \nu \Delta \varepsilon \delta o \mu \varepsilon ́ v \omega v$

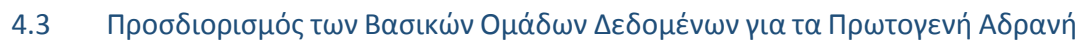

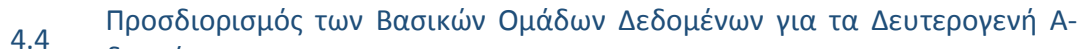
$\delta \rho \alpha v \eta ́$

4.5 П П

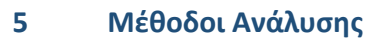

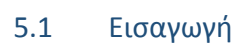

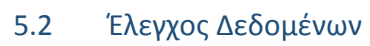

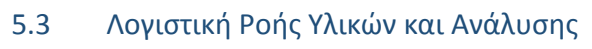

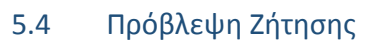

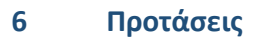

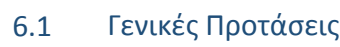

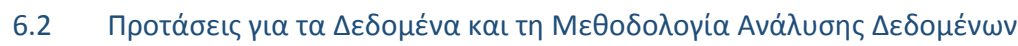

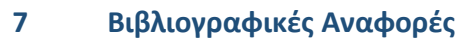

$\sum \varepsilon \lambda i \delta \alpha$ 5 9

10

18

21

22

23

25

29

30

31

33

35

36

36

37

44

47

51

52

52

55

55

61

62

63

65 


\section{Проoípı}

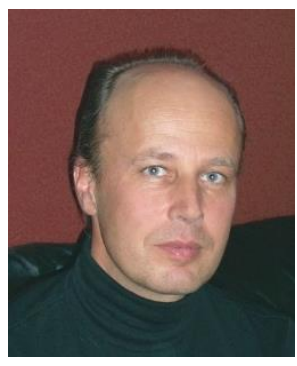

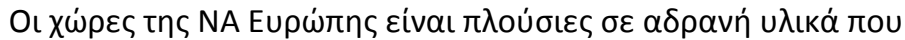

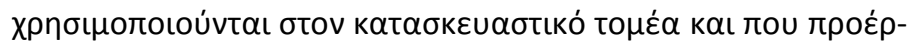

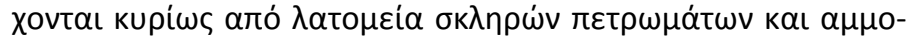

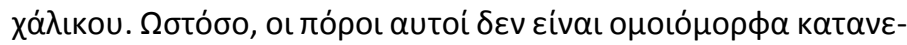

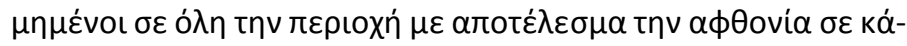

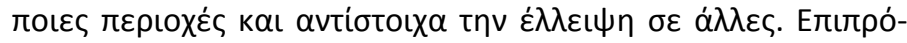

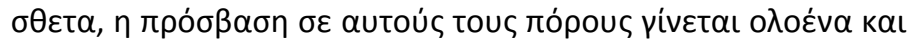

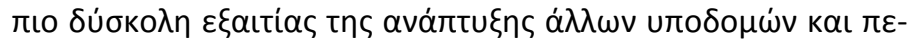

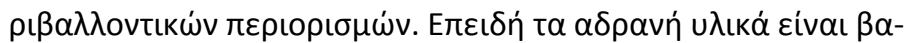

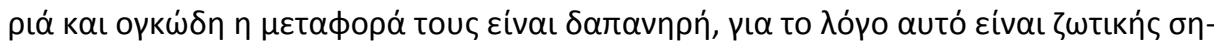

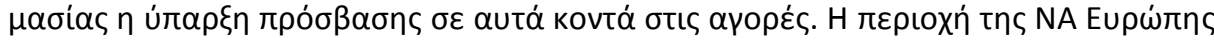

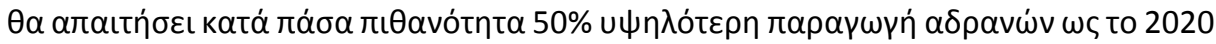

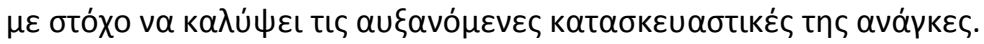

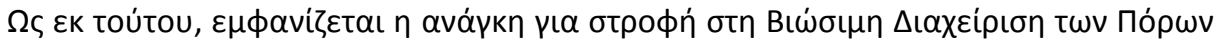

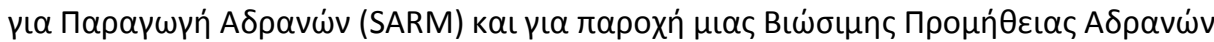

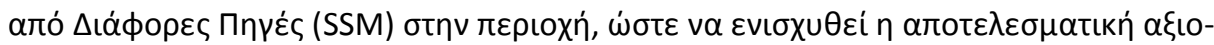

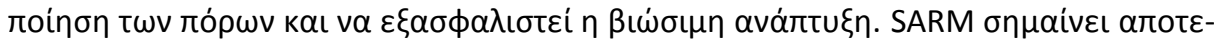

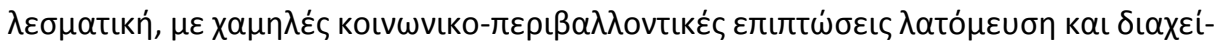

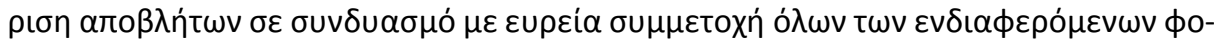

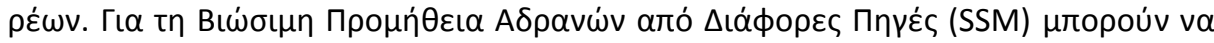

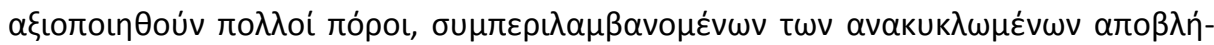

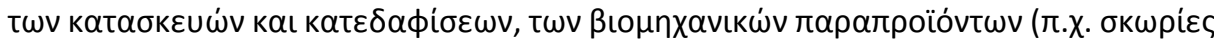

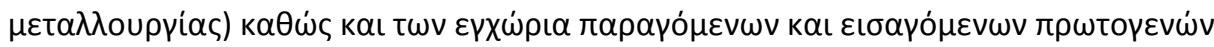

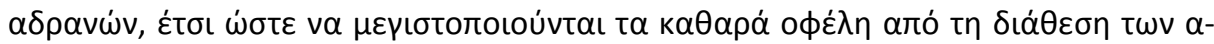

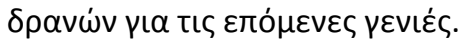

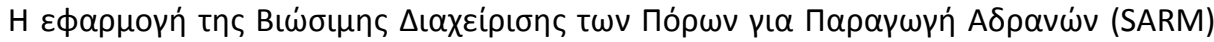

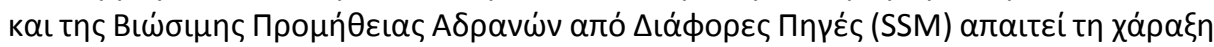

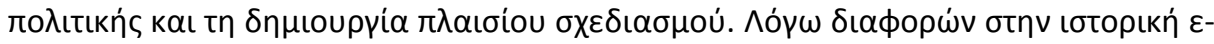

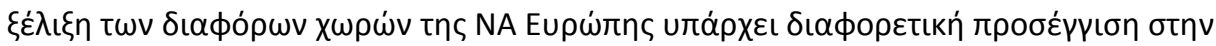

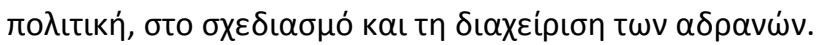

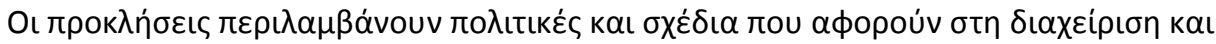

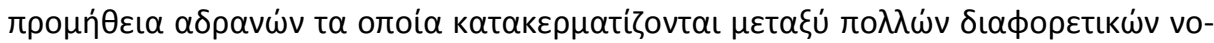

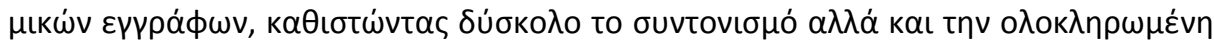

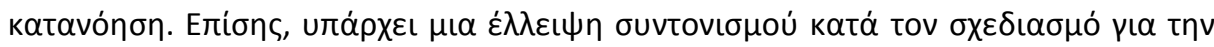

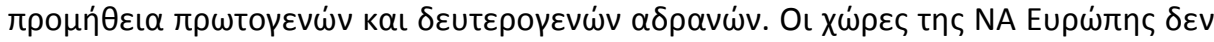

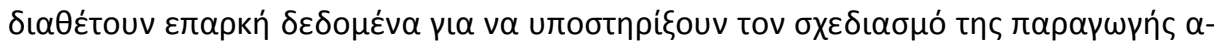

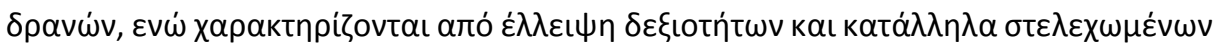




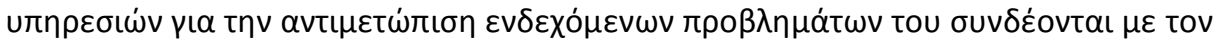

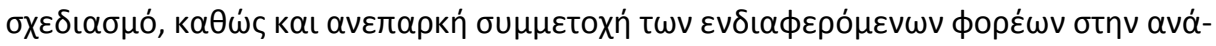

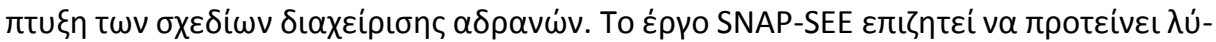

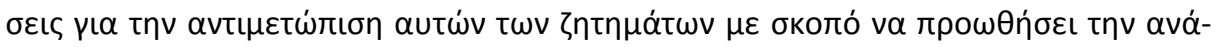

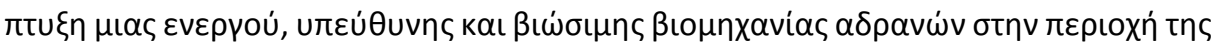
NA Eupúrn५.

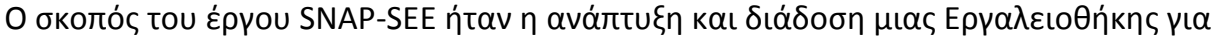

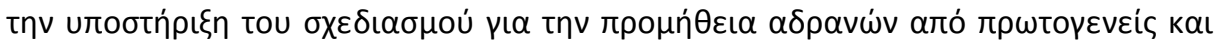

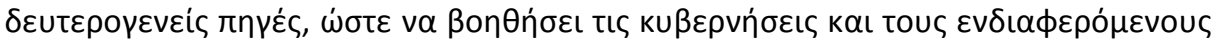

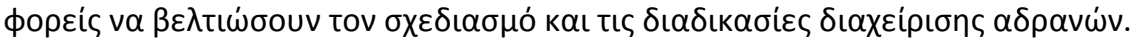

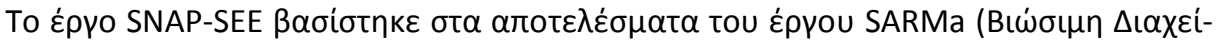

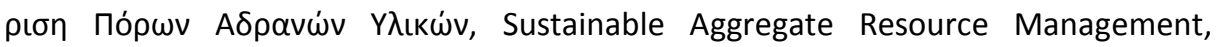

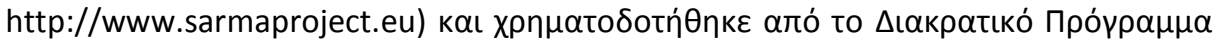

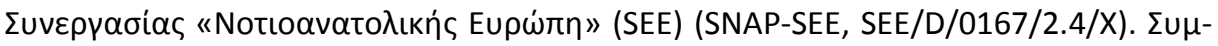

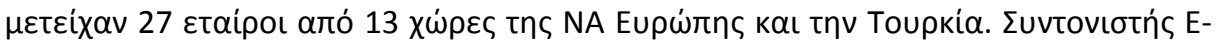

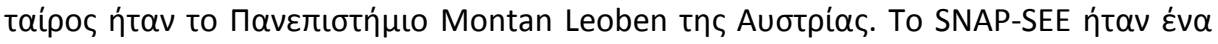

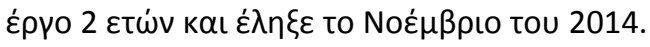

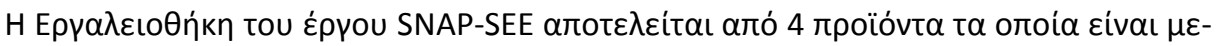
$\tau \alpha \xi u ́$ touৎ $\alpha \lambda \lambda \eta \lambda \varepsilon \dot{v} \delta \delta \varepsilon \tau \alpha$.

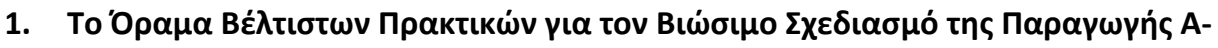

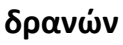

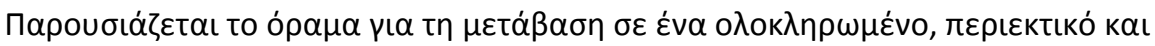

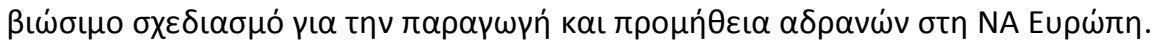

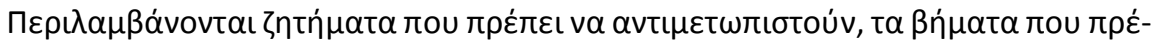

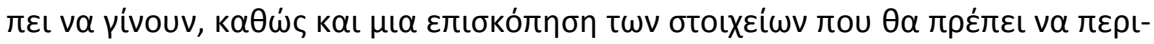

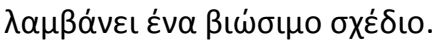

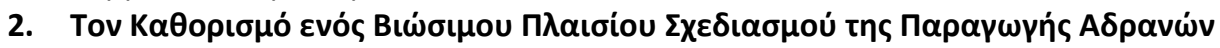

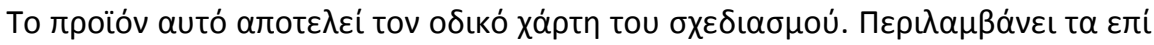

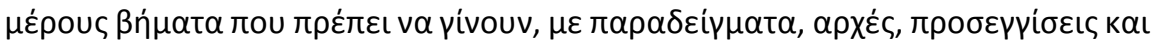

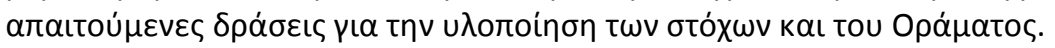

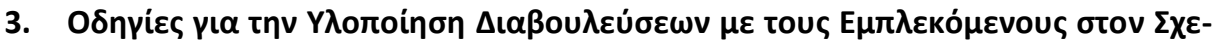

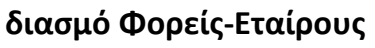

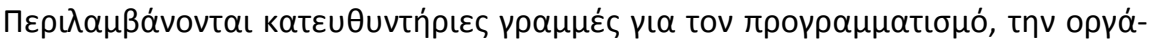

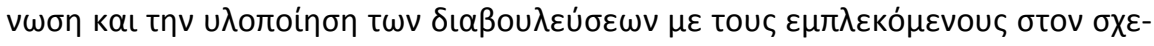

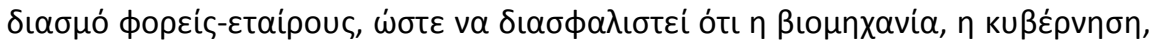

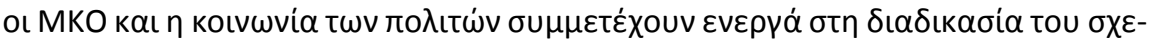

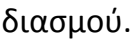




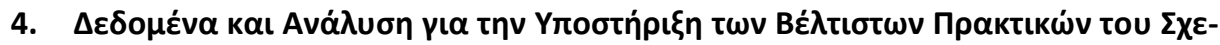

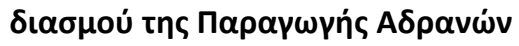

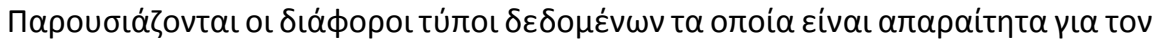

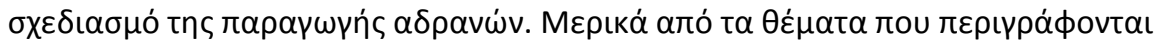

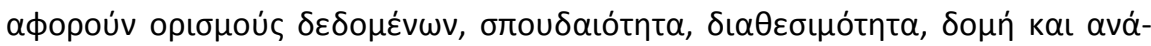

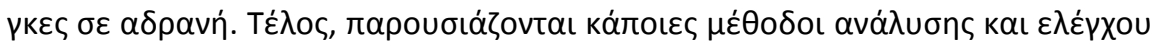

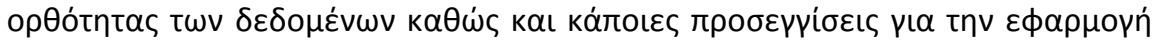

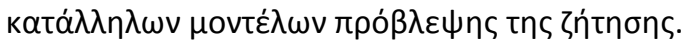

¿uvtoviotńs tou 'Eppou

Guenter Tiess

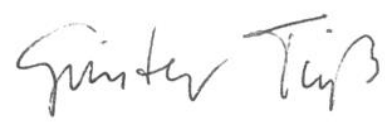

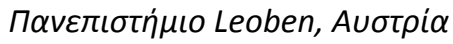




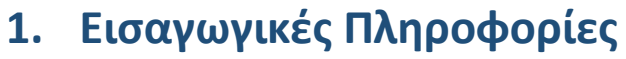

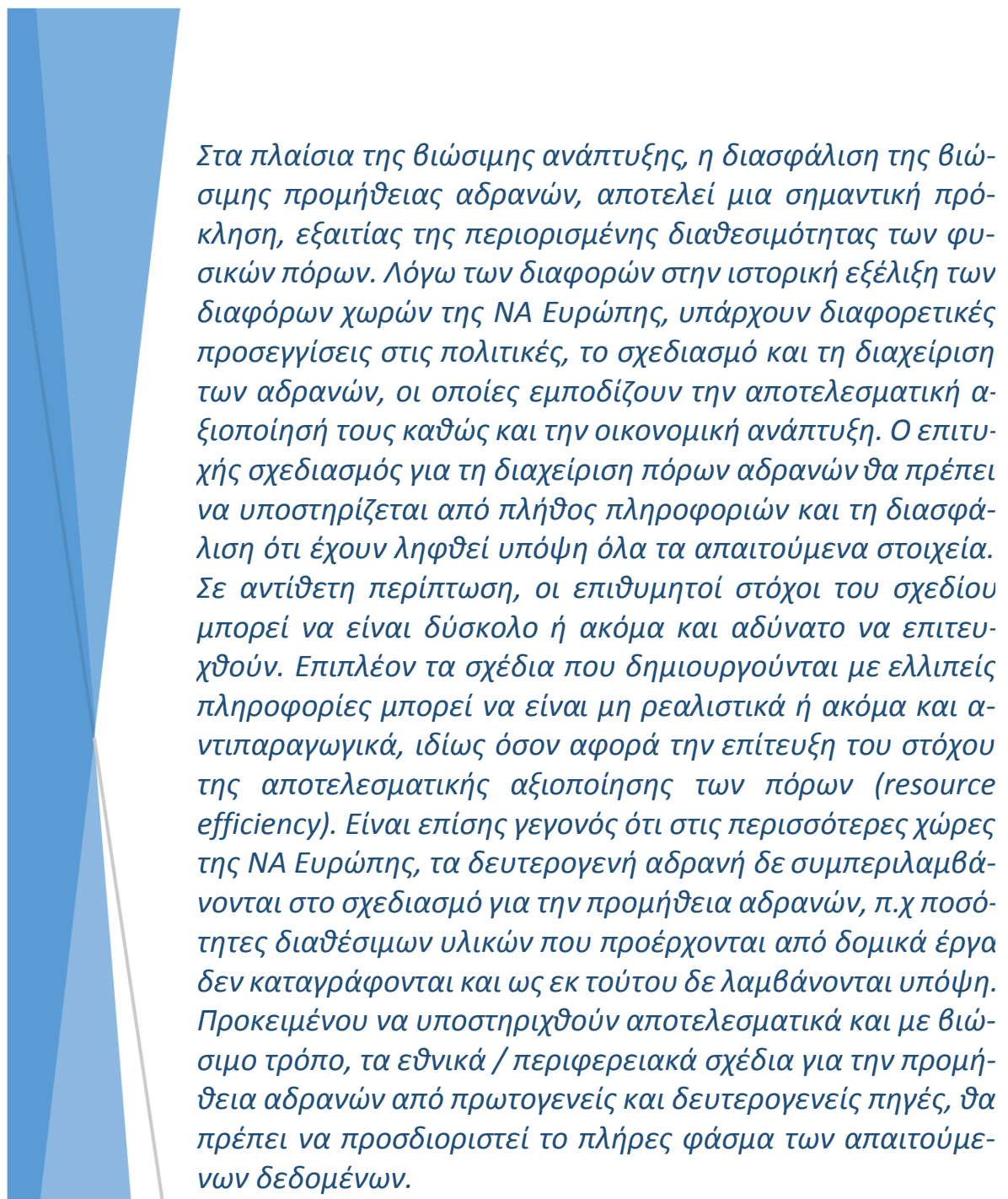




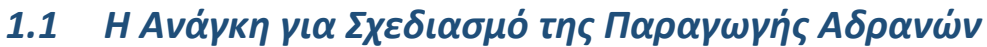

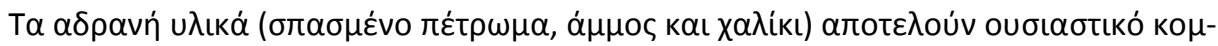

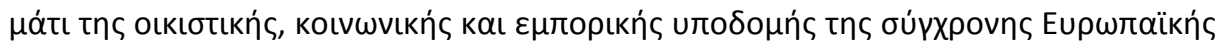

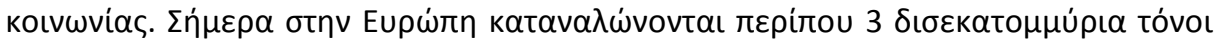

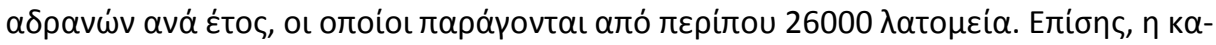

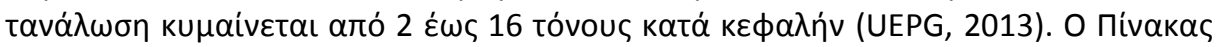

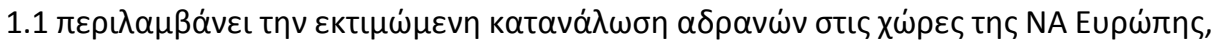

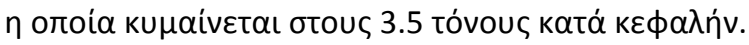

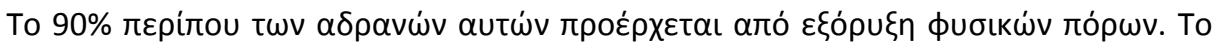

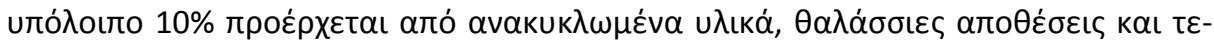

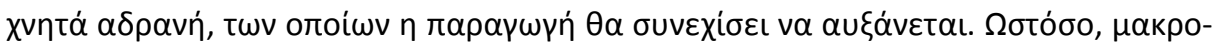

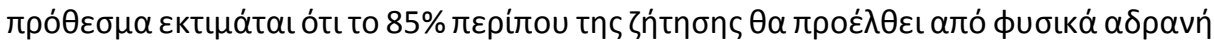

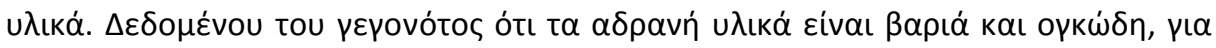

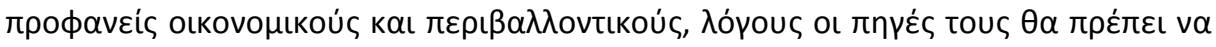

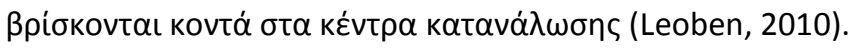

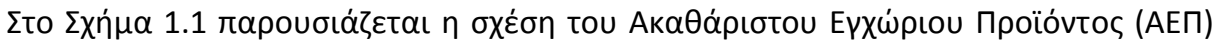

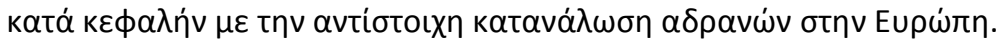

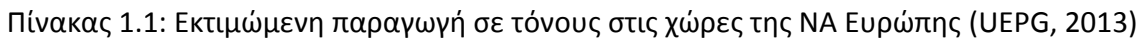

\begin{tabular}{|c|c|c|c|}
\hline & 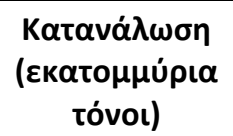 & 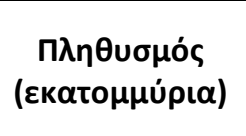 & 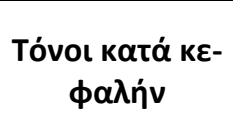 \\
\hline$A \lambda \beta \alpha v i \alpha$ & 10 & 3.2 & 3.1 \\
\hline 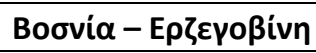 & 12 & 3.8 & 3.2 \\
\hline Bou $\lambda \gamma \alpha \rho i \alpha$ & 29 & 7.6 & 3.8 \\
\hline 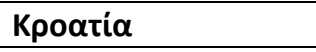 & 13 & 4.4 & 3.0 \\
\hline$E \lambda \lambda \alpha \dot{\delta} \delta \alpha$ & 25 & 11.3 & 2.2 \\
\hline Ouppapia & 36 & 10.0 & 3.6 \\
\hline FYROM & 6 & 2.0 & 3.0 \\
\hline Maupoßoúvio & 2 & 0.6 & 3.3 \\
\hline Poupavía & 96 & 22.0 & 4.4 \\
\hline$\Sigma \varepsilon \rho \beta i \alpha$ & 17 & 7.3 & 2.3 \\
\hline$\Sigma \lambda о \beta \alpha \kappa i \alpha$ & 23 & 5.4 & 4.3 \\
\hline$\Sigma \lambda o \beta \varepsilon v i \alpha$ & 9 & 2.0 & 4.5 \\
\hline$\Sigma u ́ v o \lambda \alpha$ & 278 & 79.6 & 3.5 \\
\hline
\end{tabular}




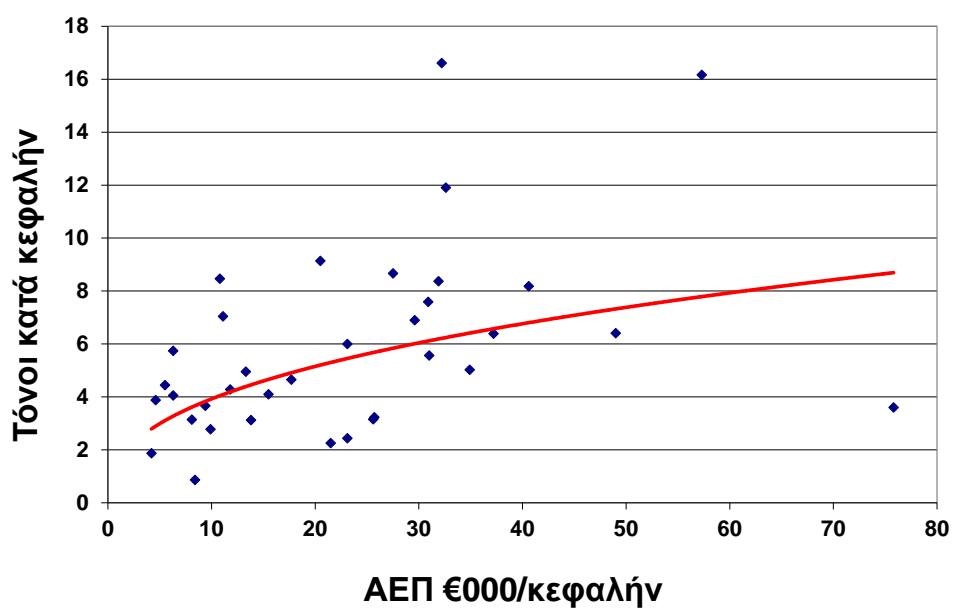

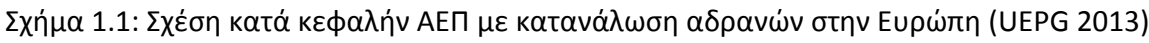

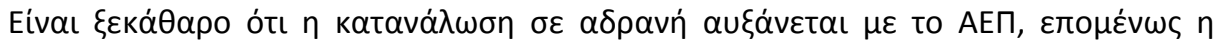

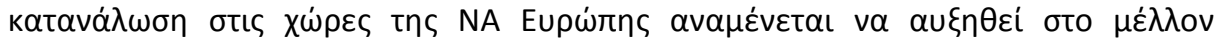

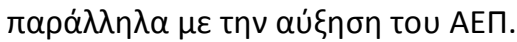

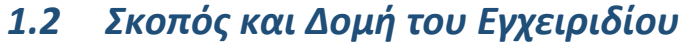

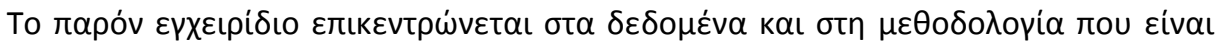

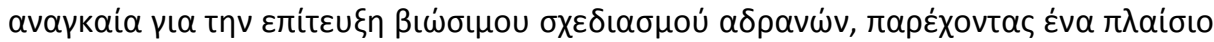

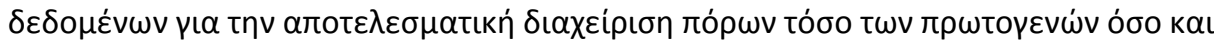

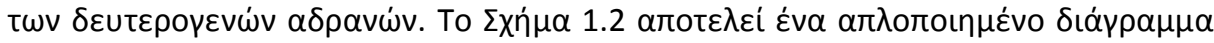

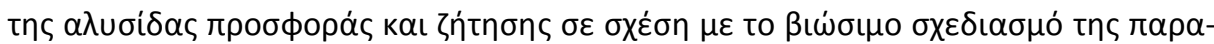

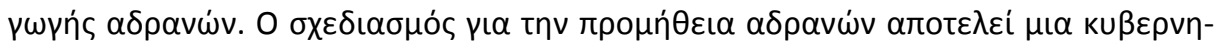

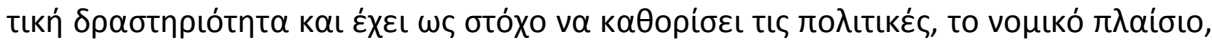

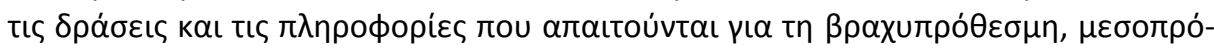

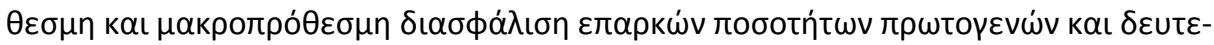

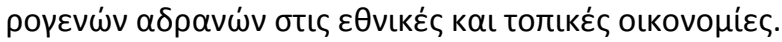

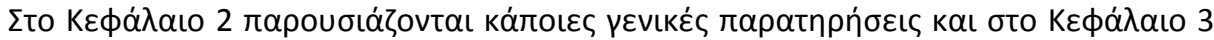

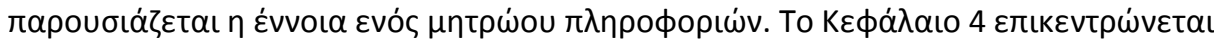

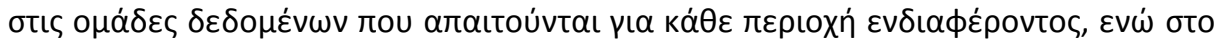

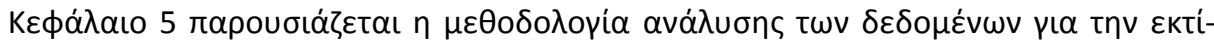

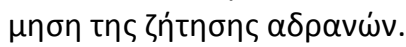




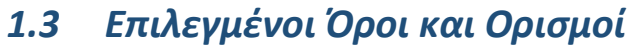

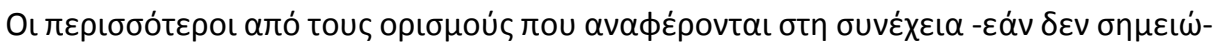

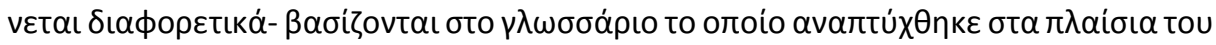
ह́pyou SARMa (SARMa, 2011).

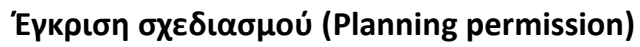

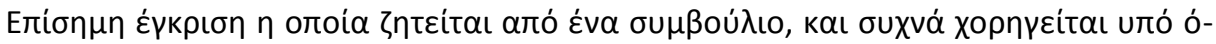

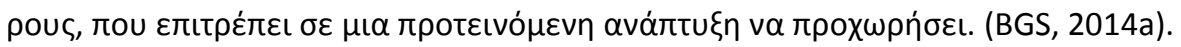

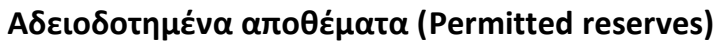

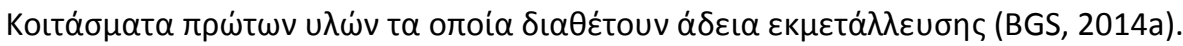

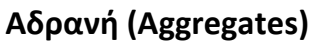

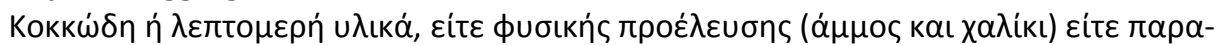

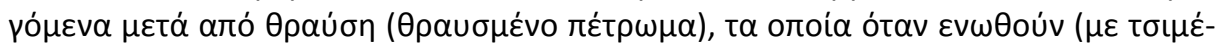

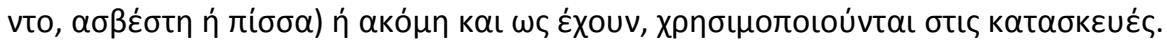

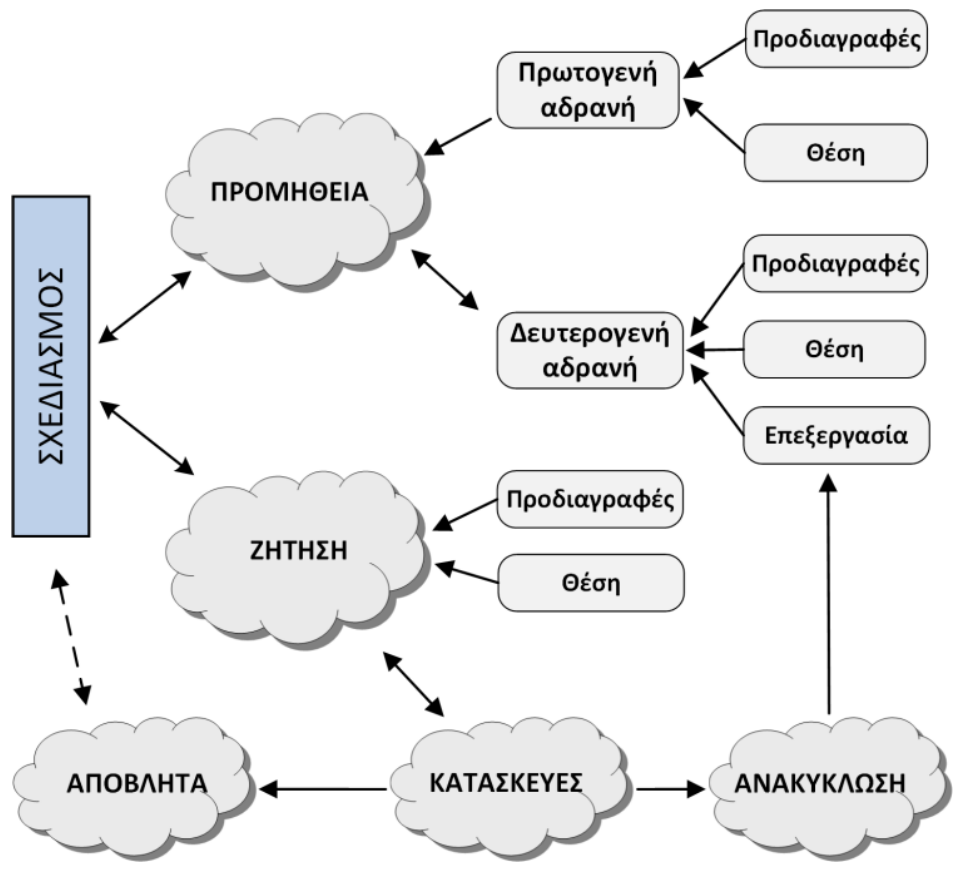

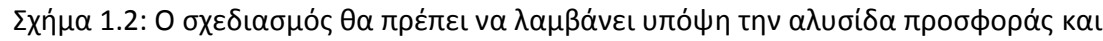

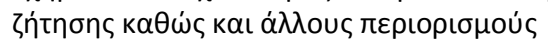




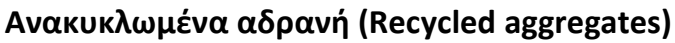

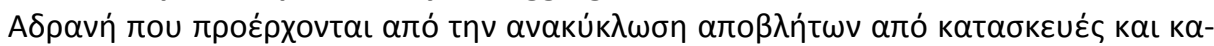

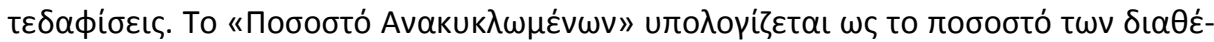

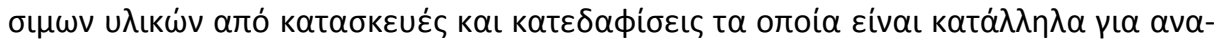
кúk $\lambda \omega \sigma \eta$.

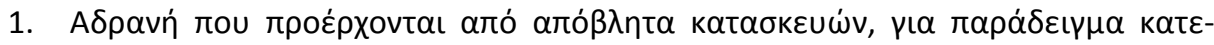

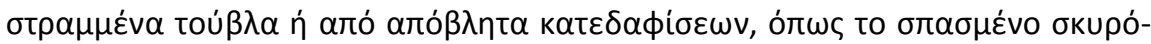

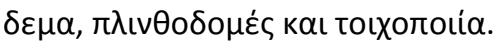

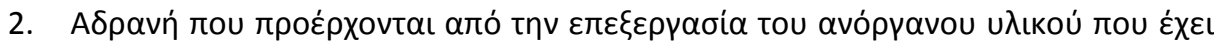

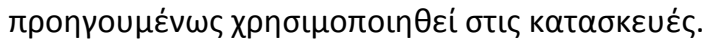

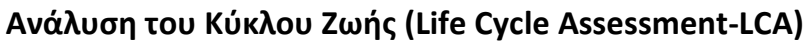

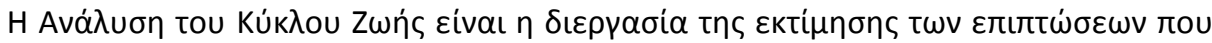

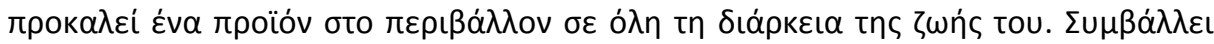

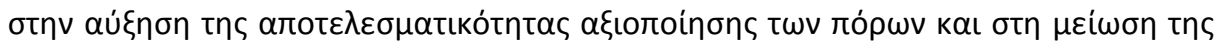

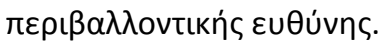

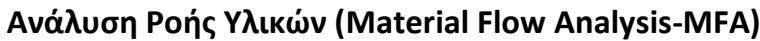

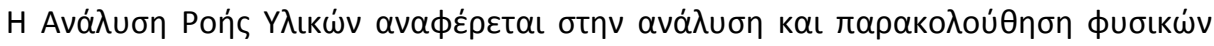

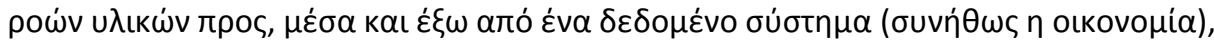

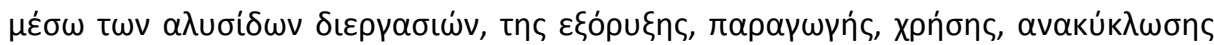

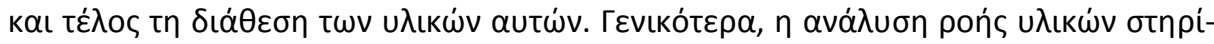

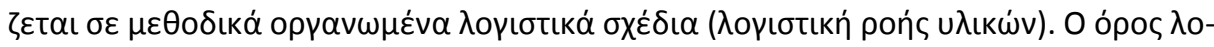

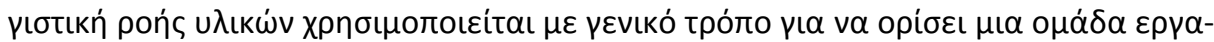

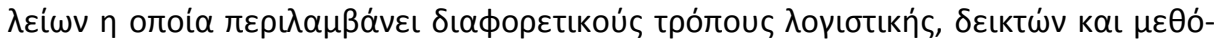

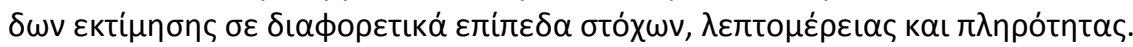

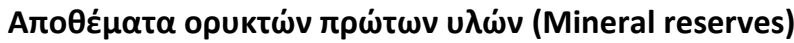

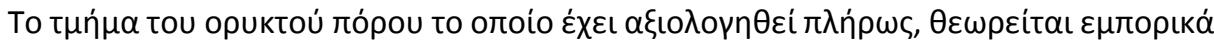

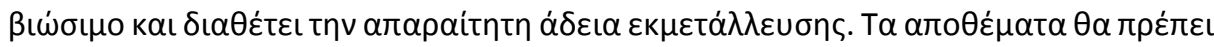

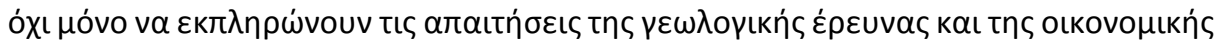

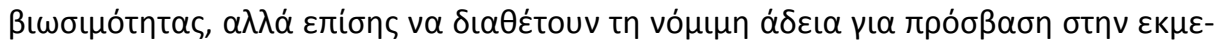

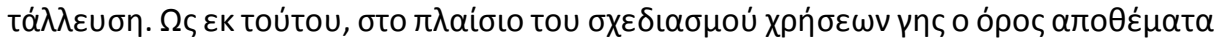

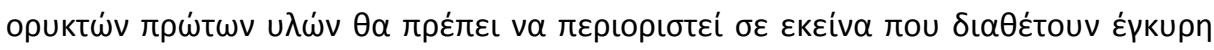

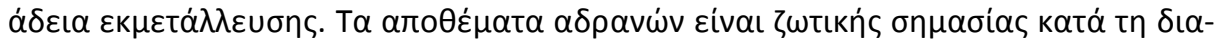

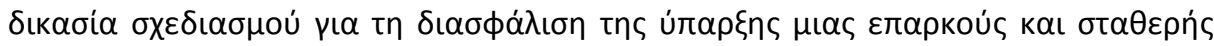

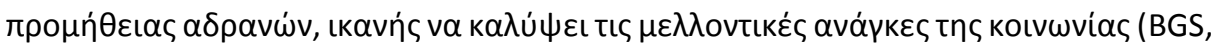
2014c) 


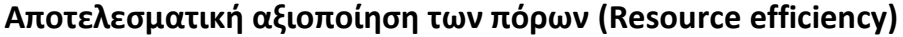

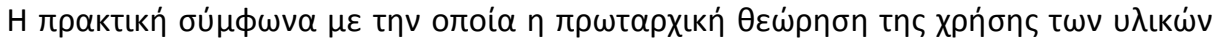

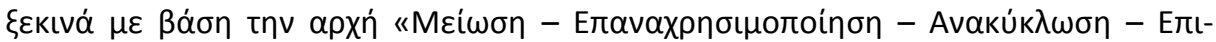

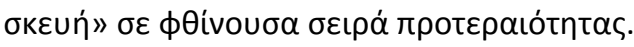

\section{Apxń (Authority)}

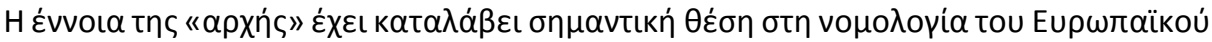

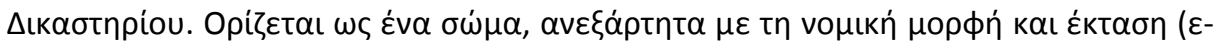

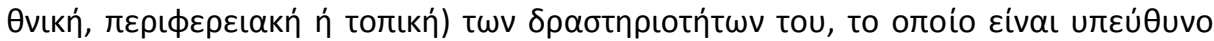

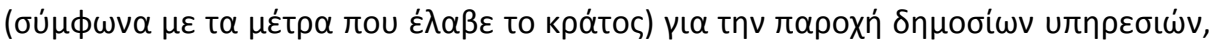

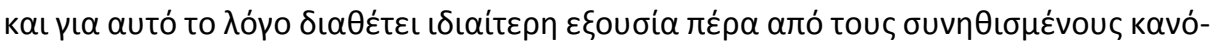

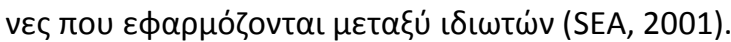

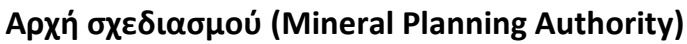

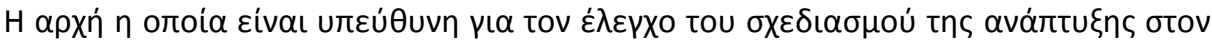

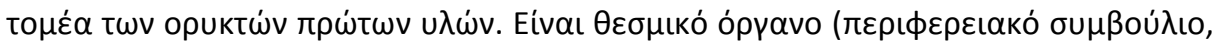

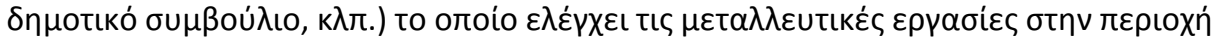

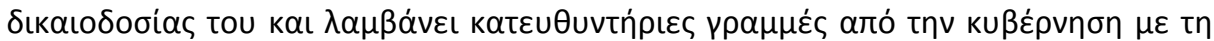

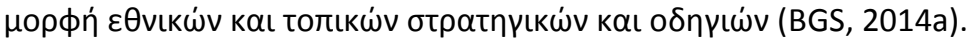

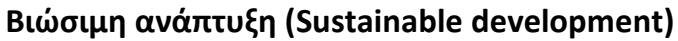

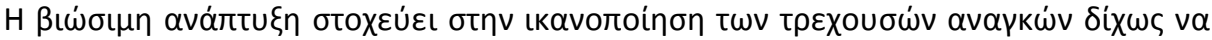

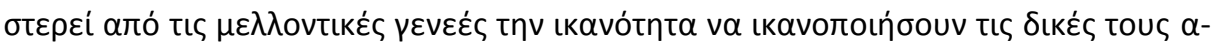

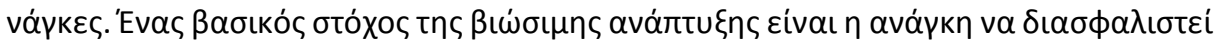

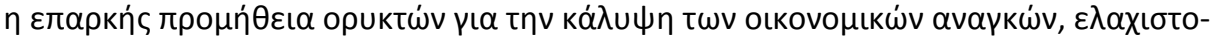

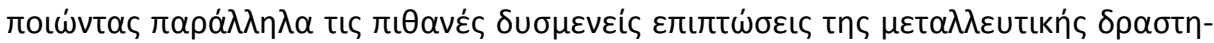

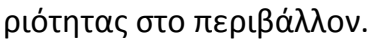

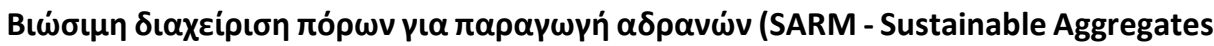
Resource Management)

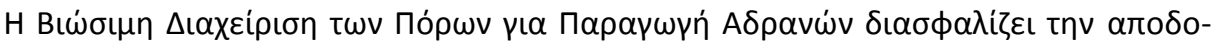

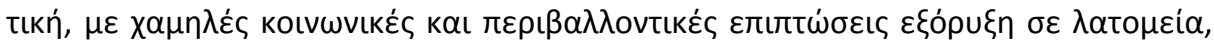

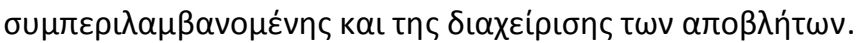

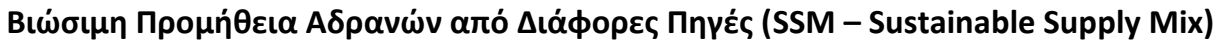

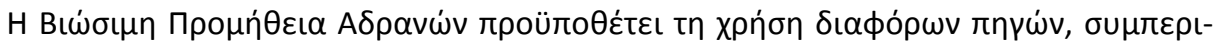

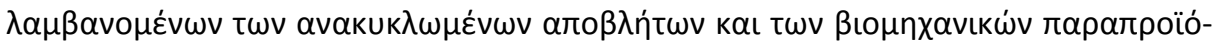

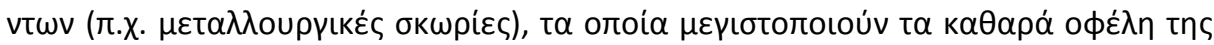

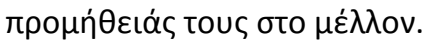




\section{$\Delta \alpha v \varepsilon ı \theta \dot{\alpha} \lambda \alpha \mu \mathrm{ol}$ (Borrow pits)}

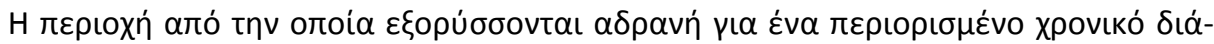

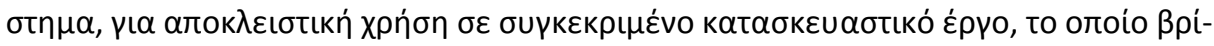

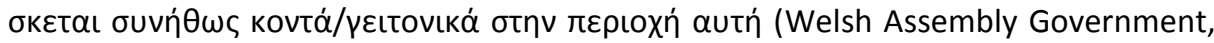
2011).

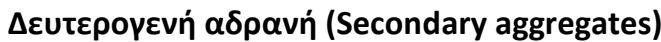

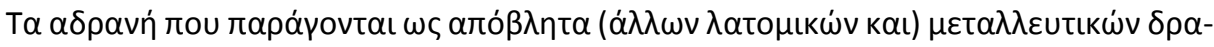

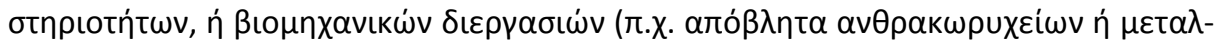

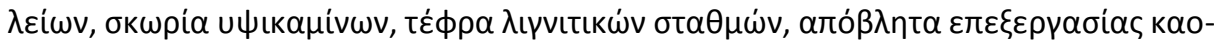

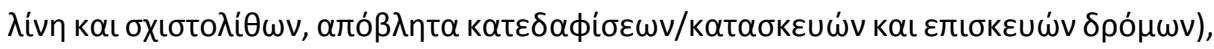

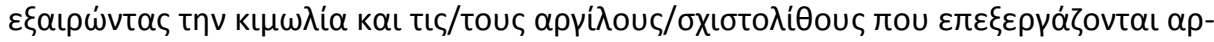

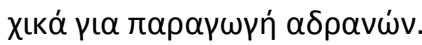

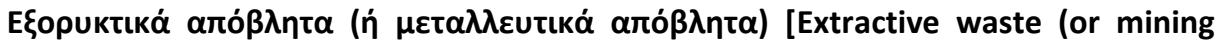 waste)]}

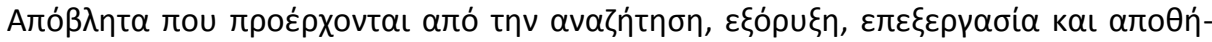

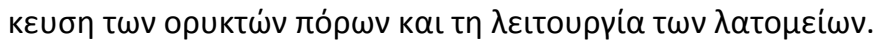

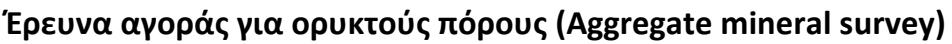

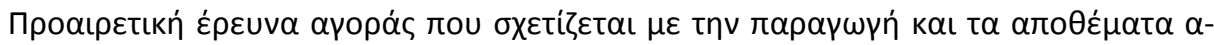

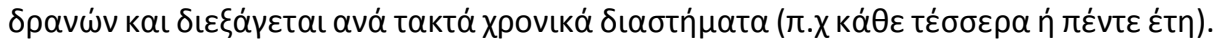

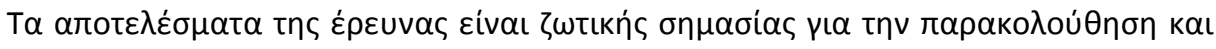

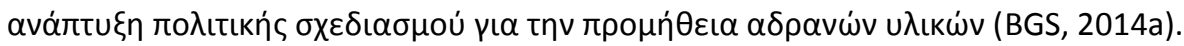

\section{Zńtnon (Demand)}

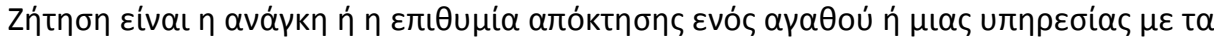

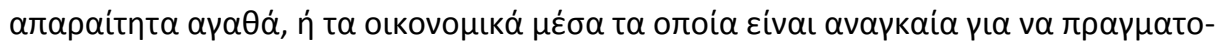

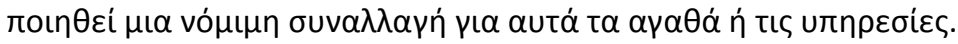

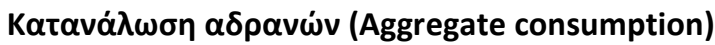

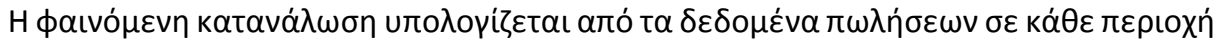

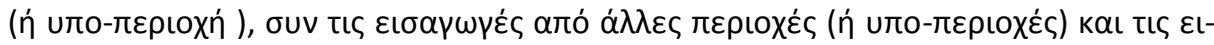

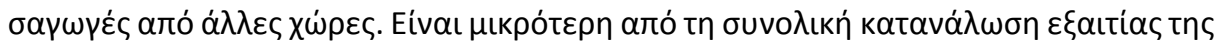

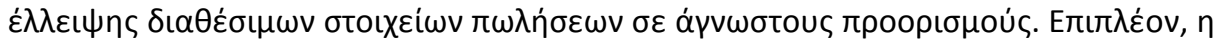

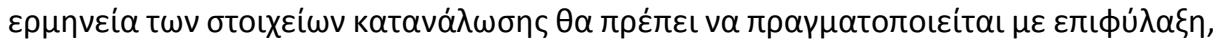

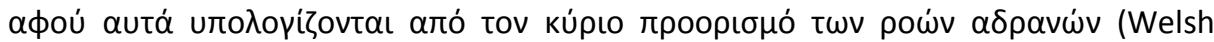
Assembly Government, 2011). 


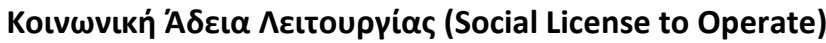

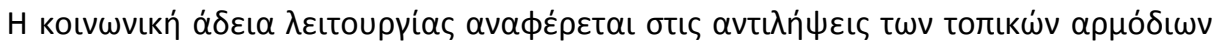

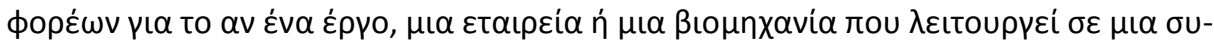

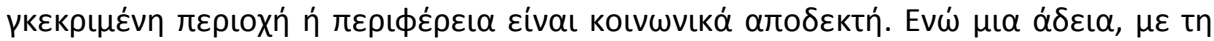

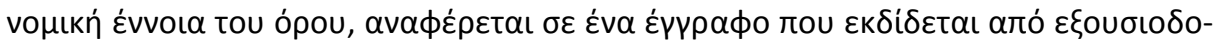

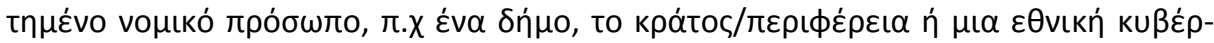

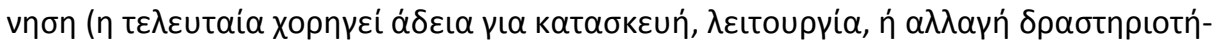

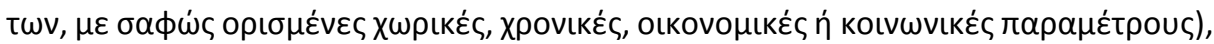

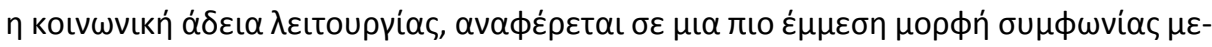

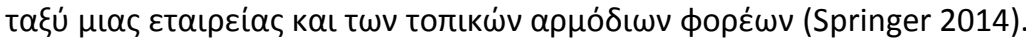

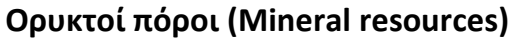

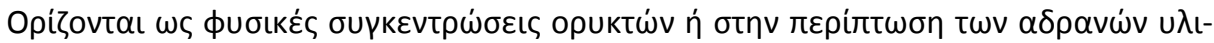

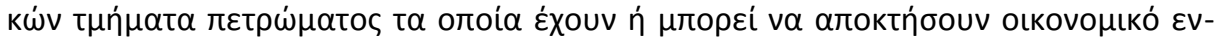

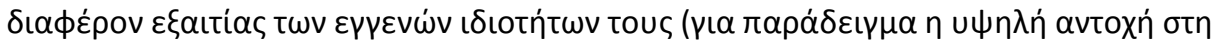

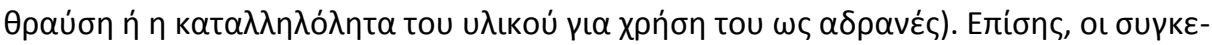

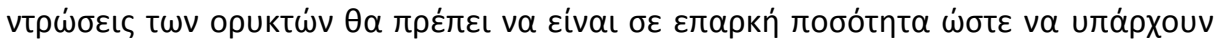

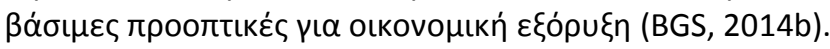

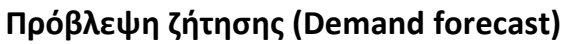

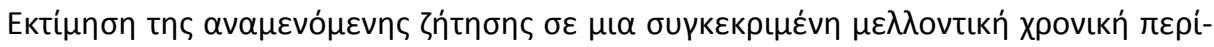
oঠo.

\section{Пробфора́ (Supply)}

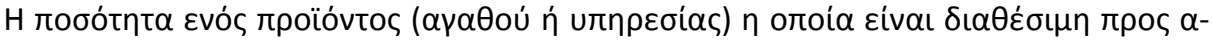

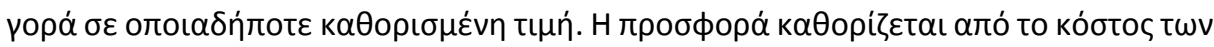

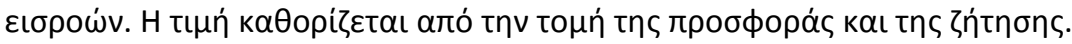

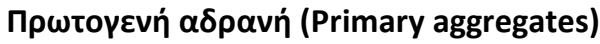

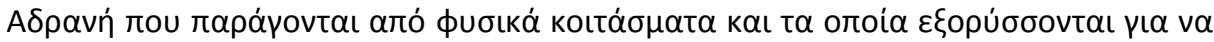

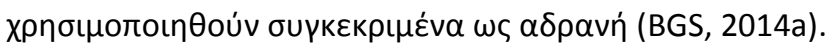

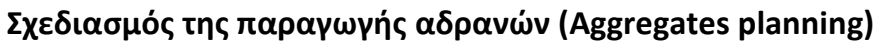

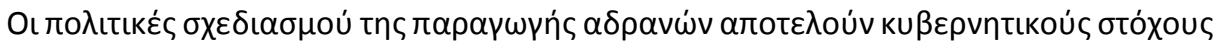

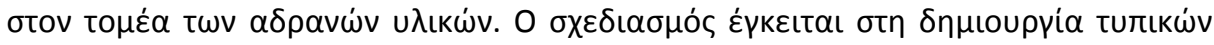

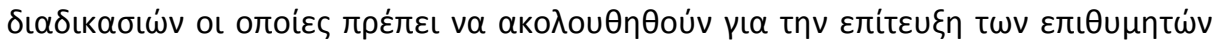

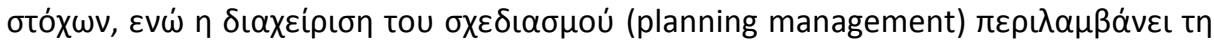

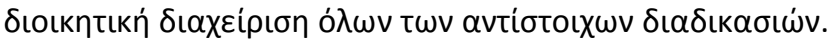




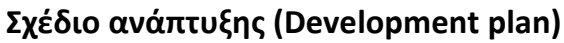

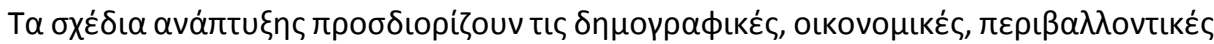

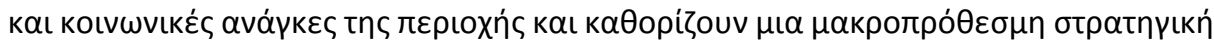

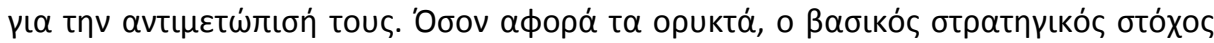

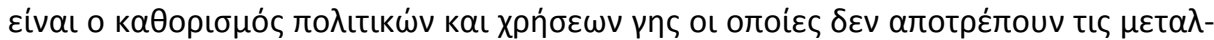

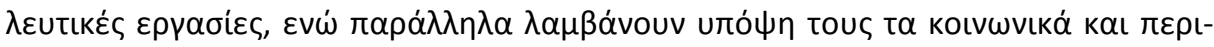

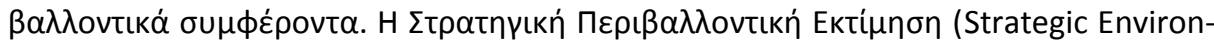

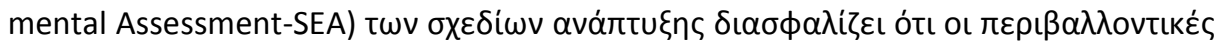

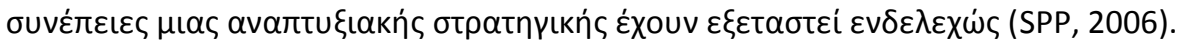

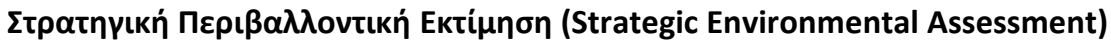

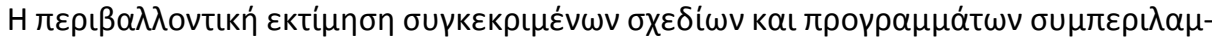

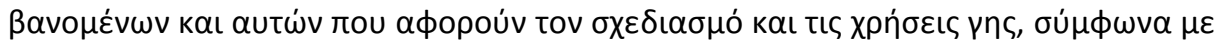

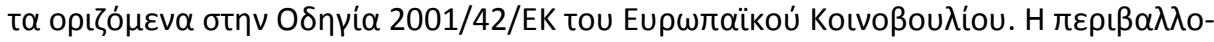

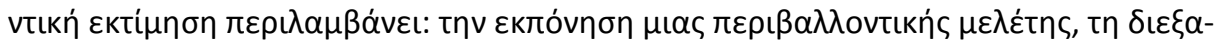

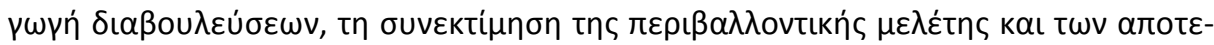

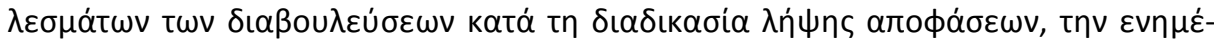


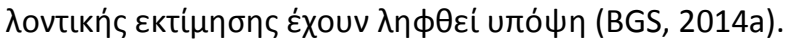

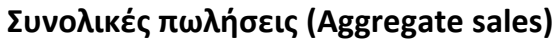

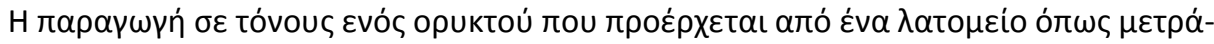

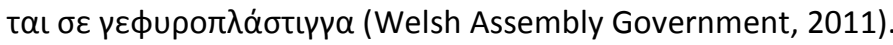

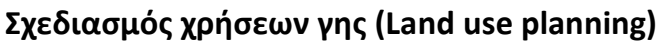

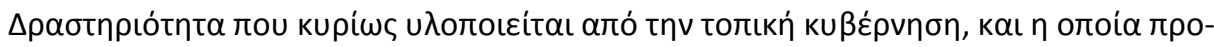

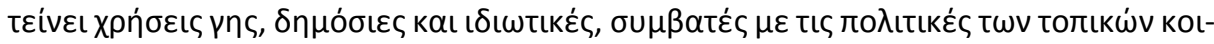

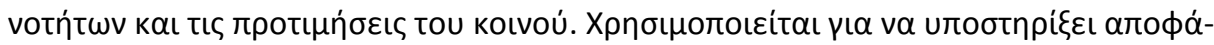

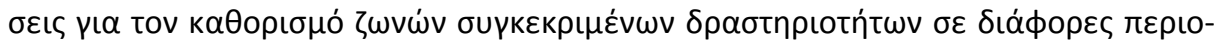
$\chi \varepsilon ́ \varsigma$.

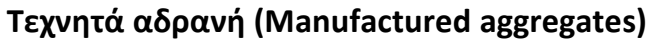

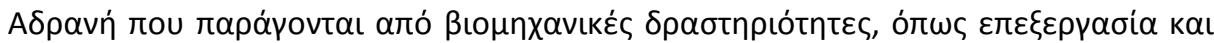

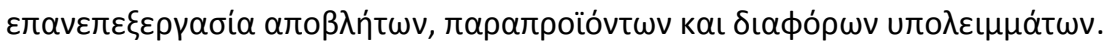

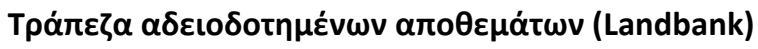

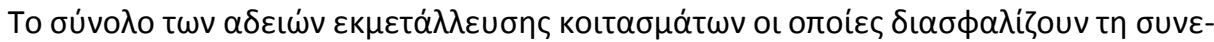

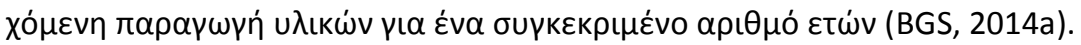

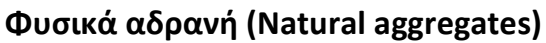

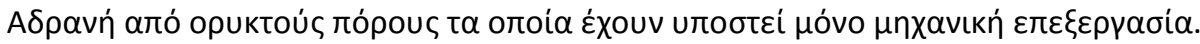




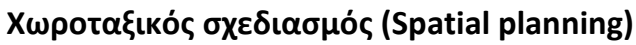

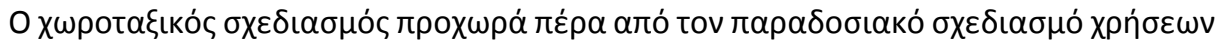

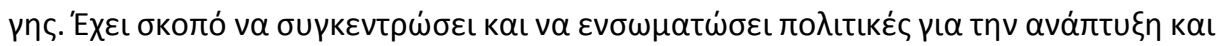

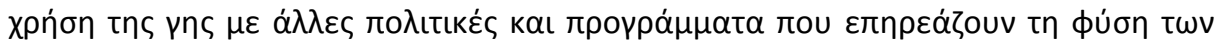

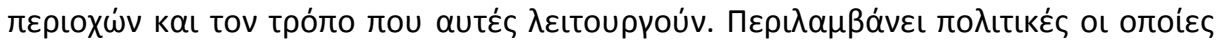

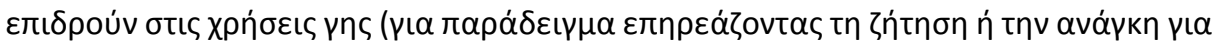

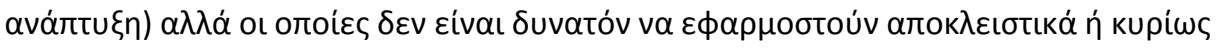

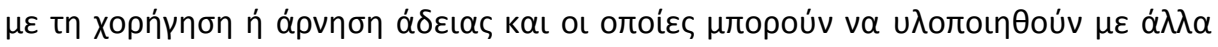
$\mu \varepsilon ́ \sigma \alpha$ (BGS, 2014a).

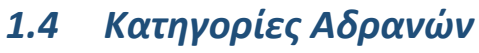

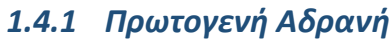

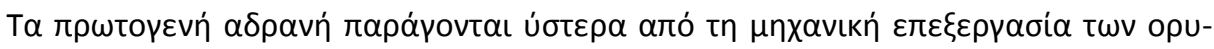

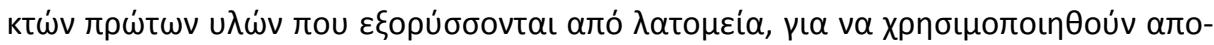

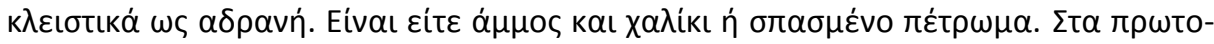

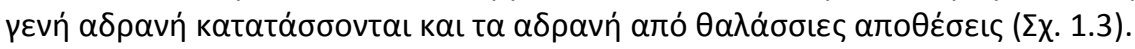

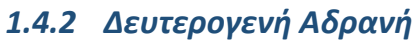

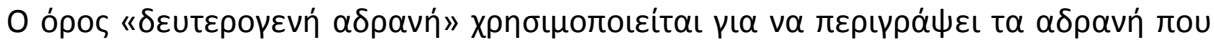

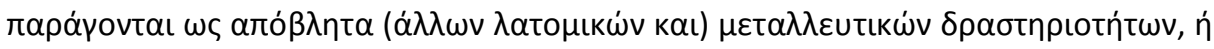

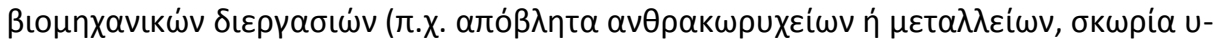

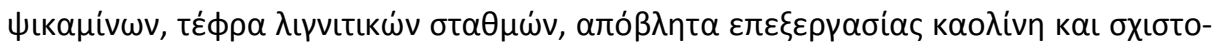

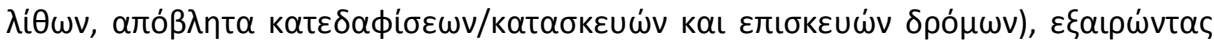

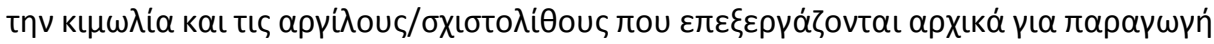
$\alpha \delta \rho \alpha v \omega \dot{v}(\Sigma \chi .1 .3)$.

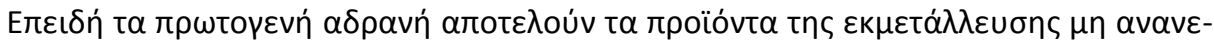

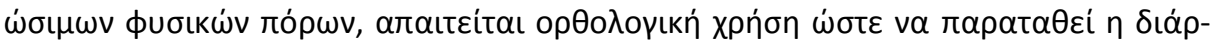

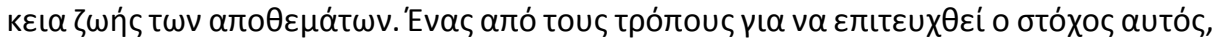

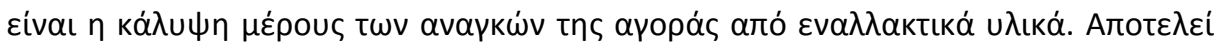

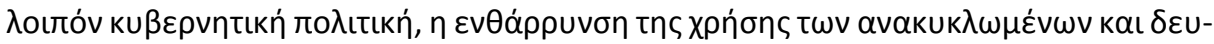

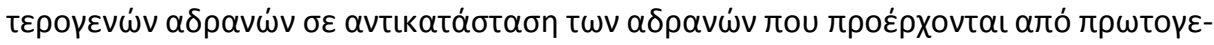

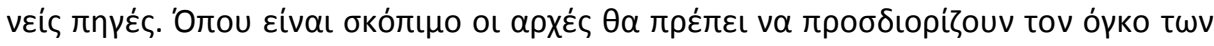

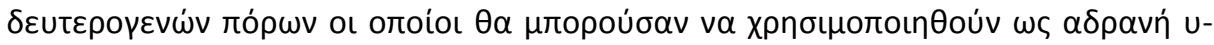

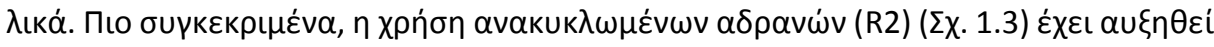

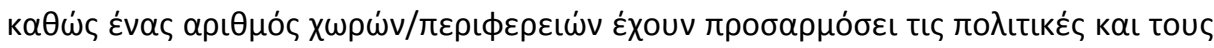

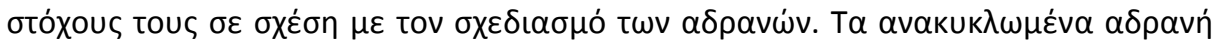

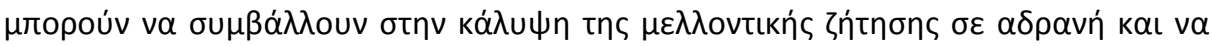




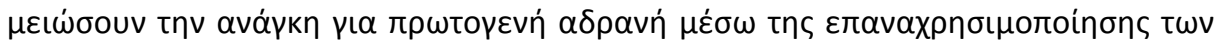

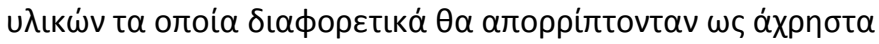

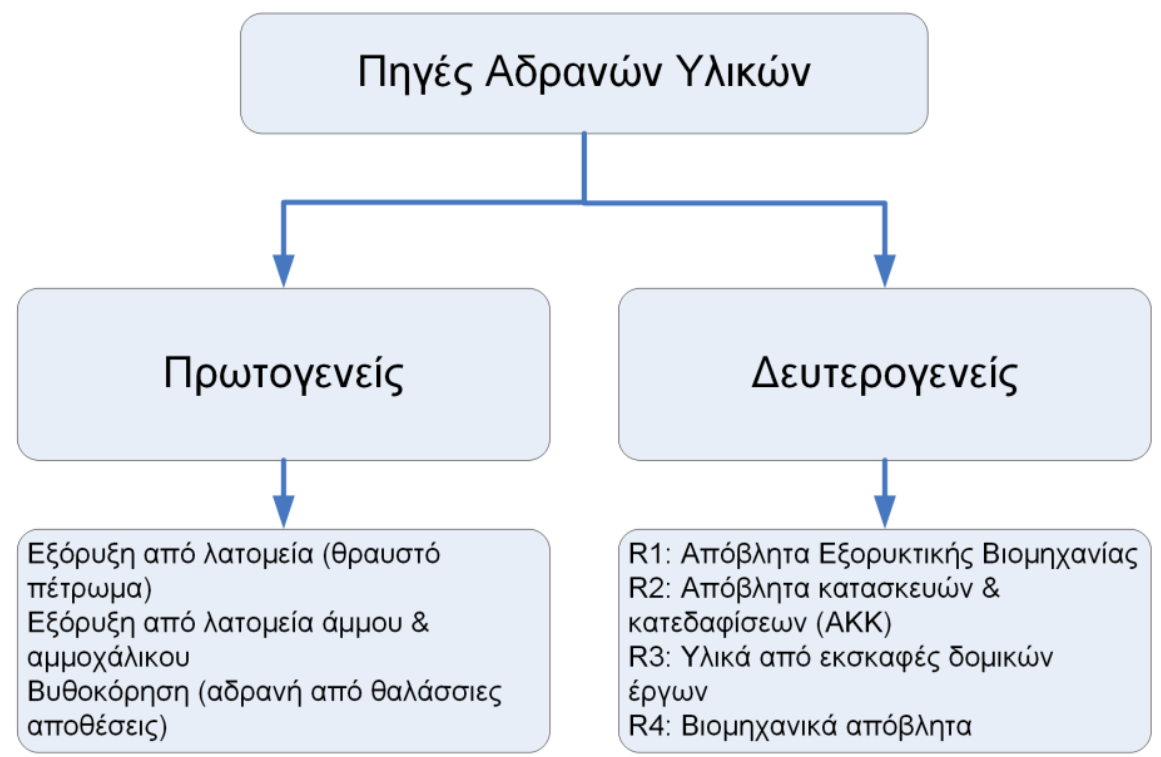

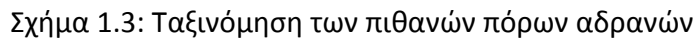


20 


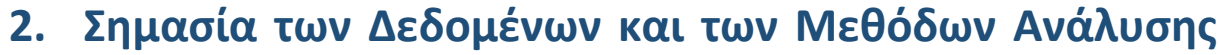

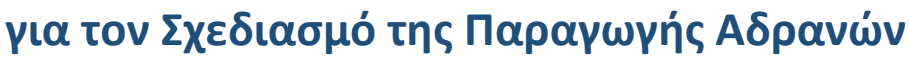

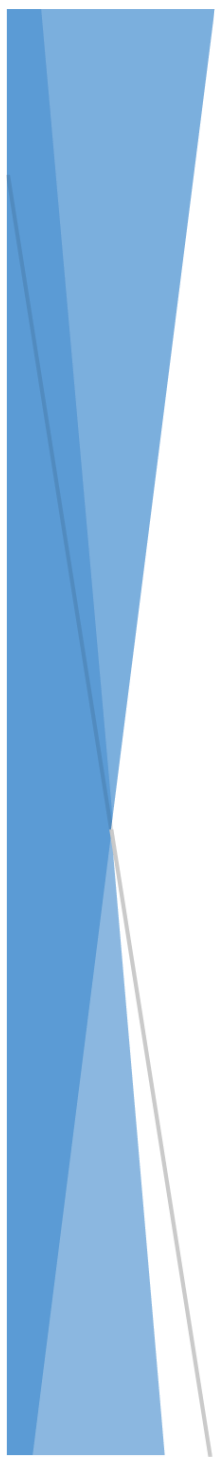

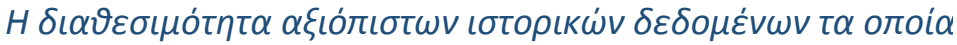

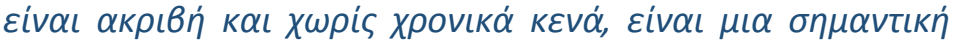

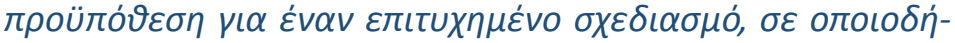

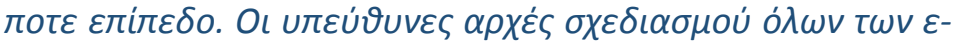

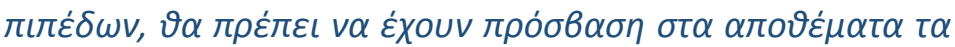

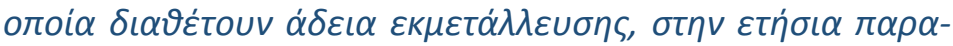

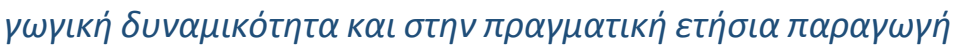

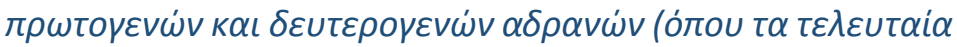

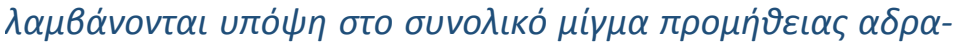

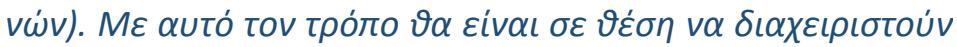

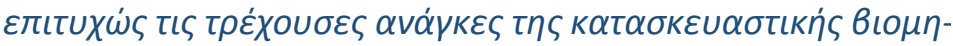

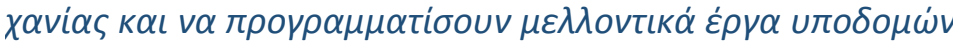

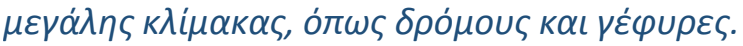




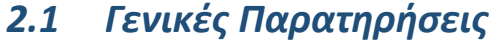

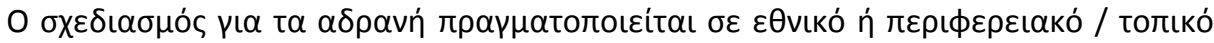

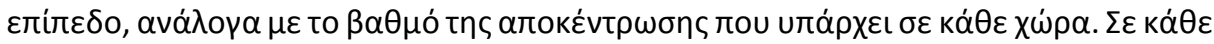

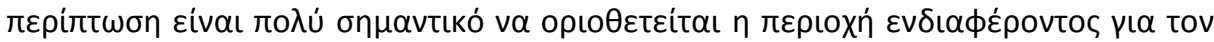

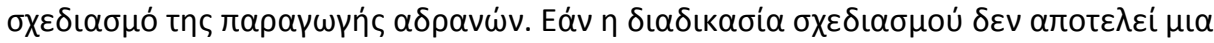

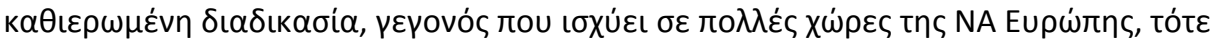

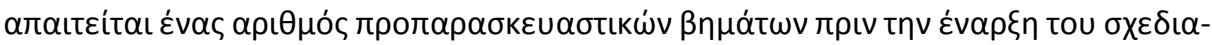

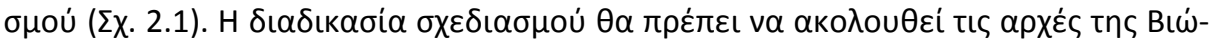

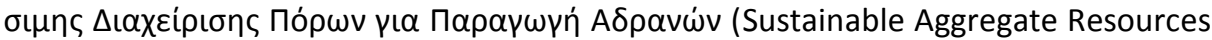

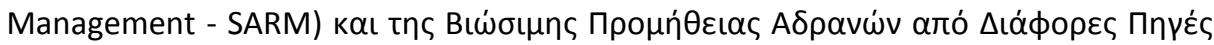

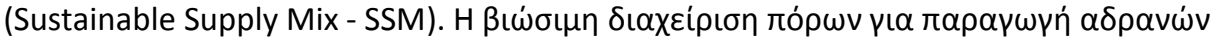

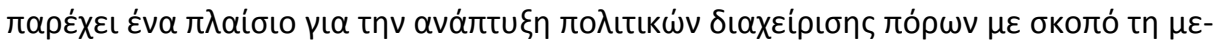

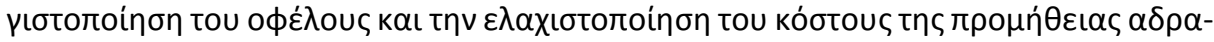

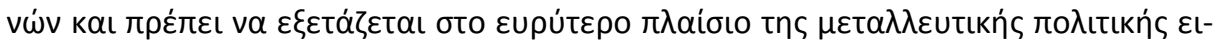

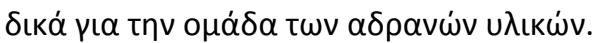

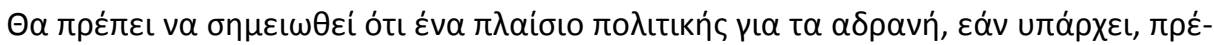

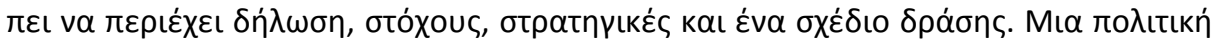

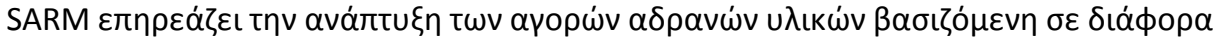

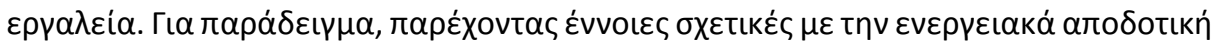

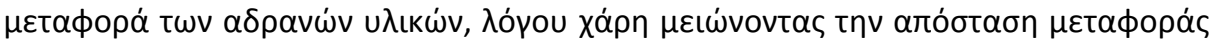

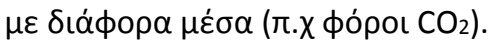

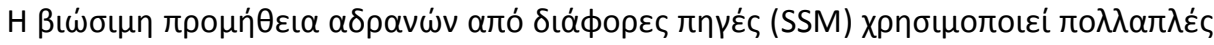

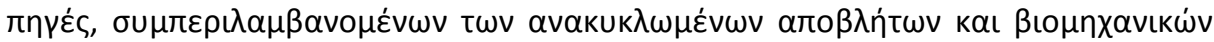

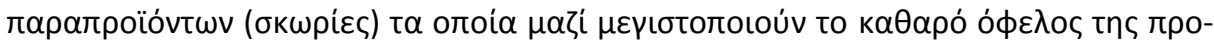

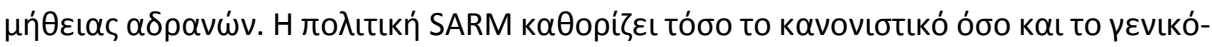

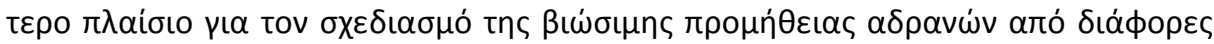

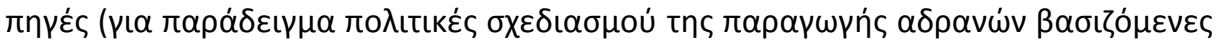

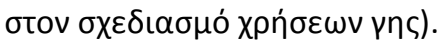

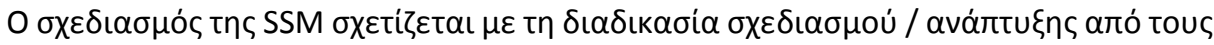

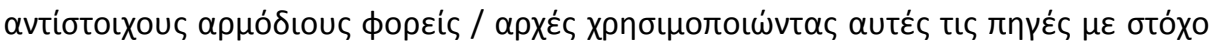

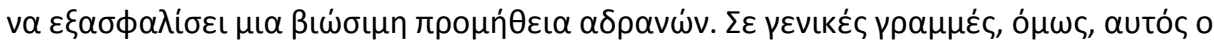

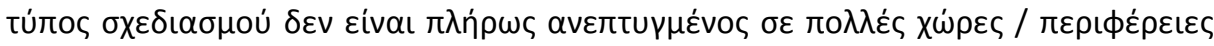

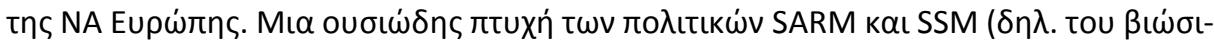

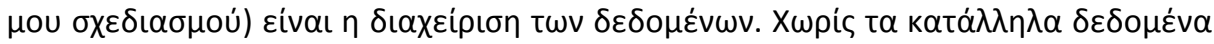

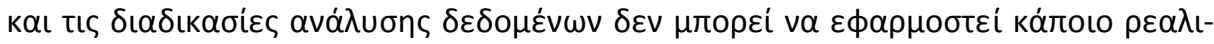

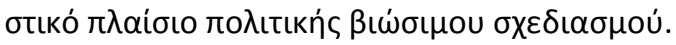




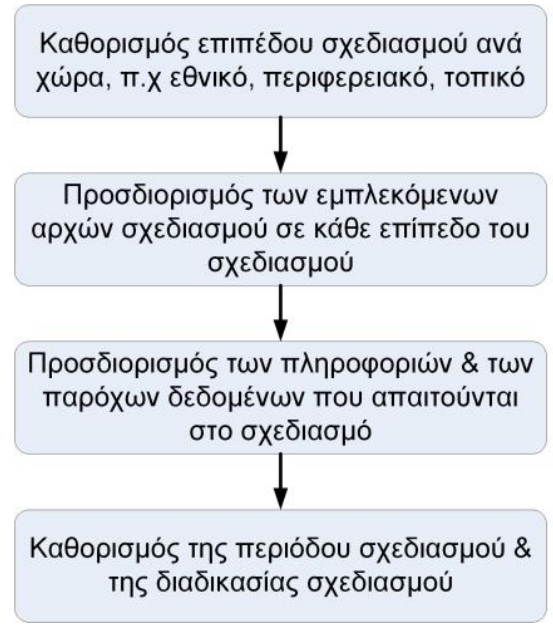

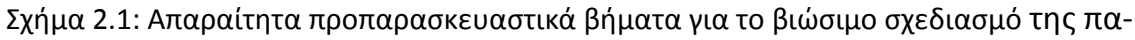

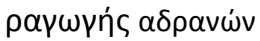

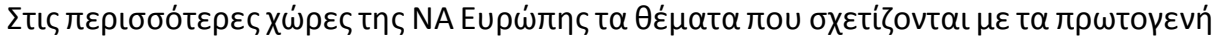

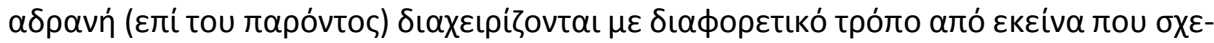

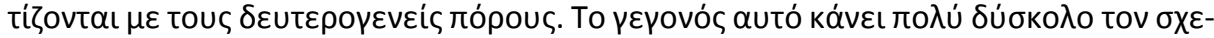

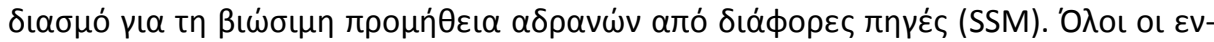

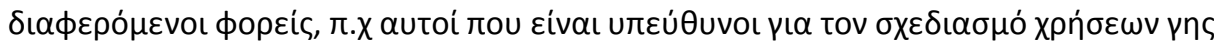

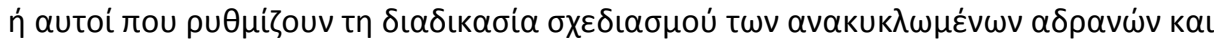

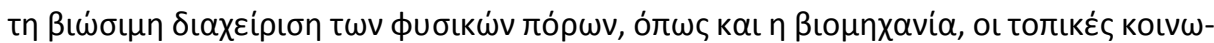

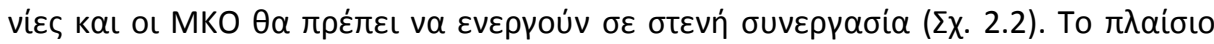

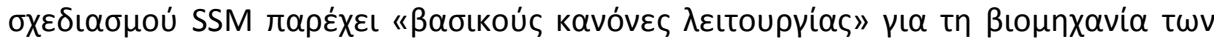

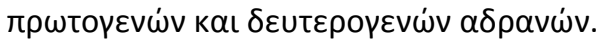

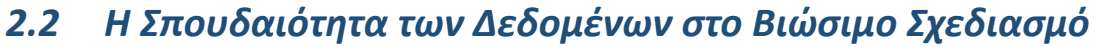

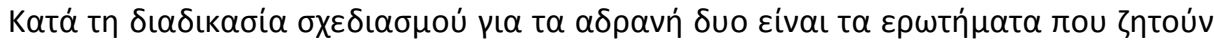

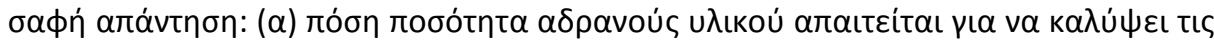

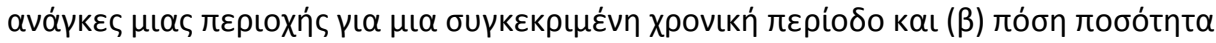

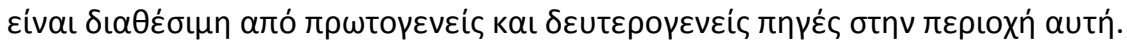

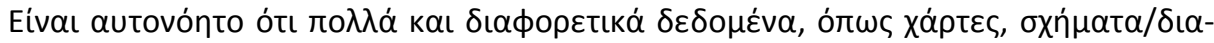

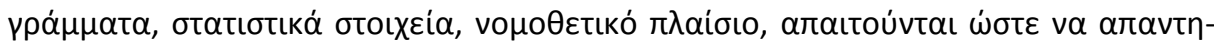

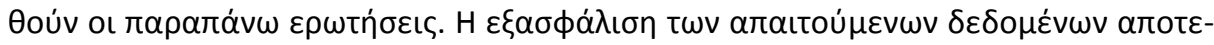

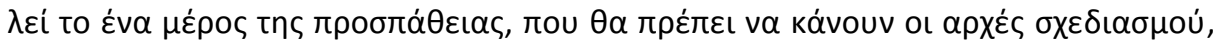




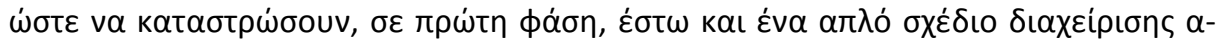

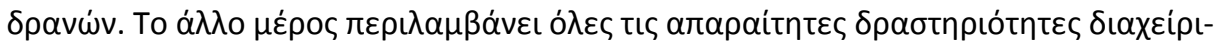

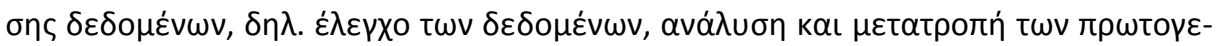

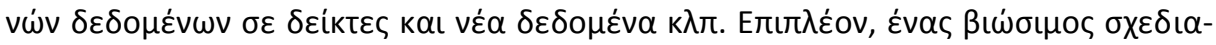

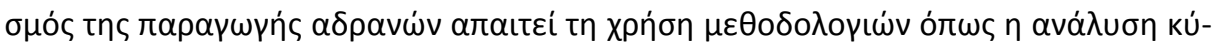

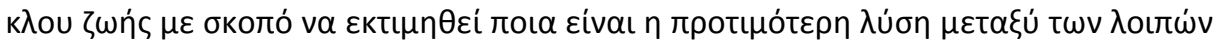

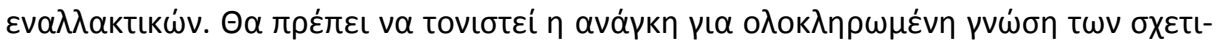

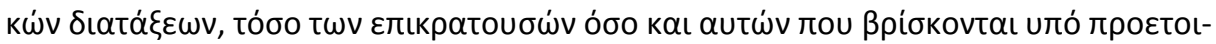

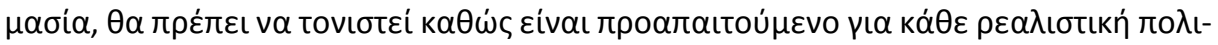

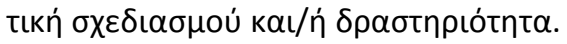

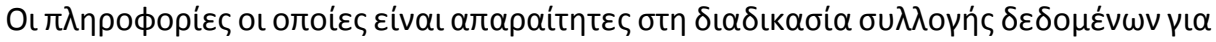

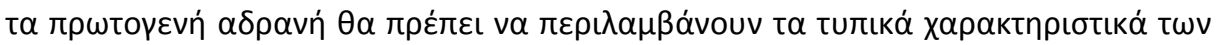

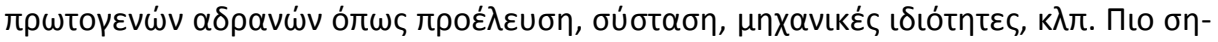

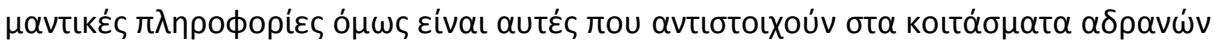

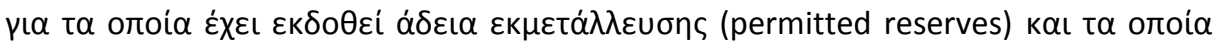

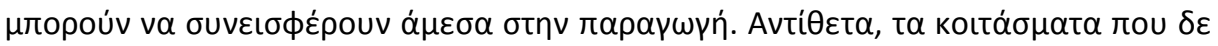

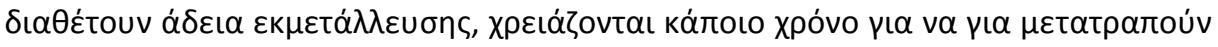

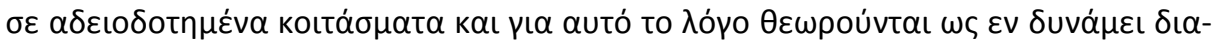

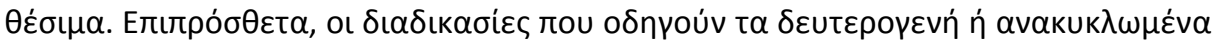

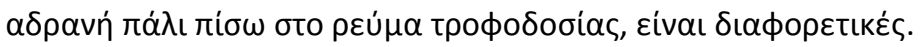

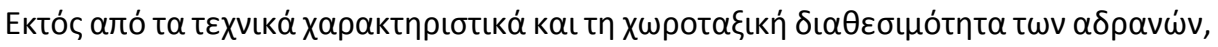

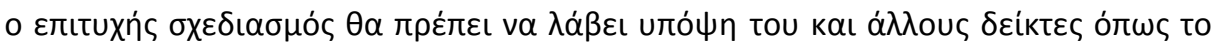

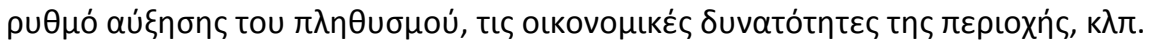

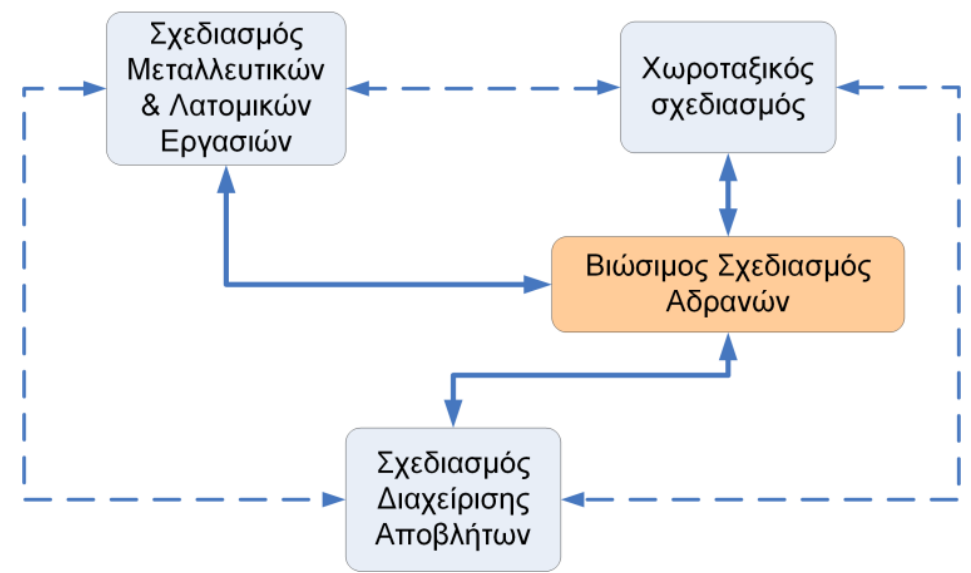

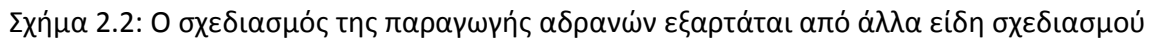




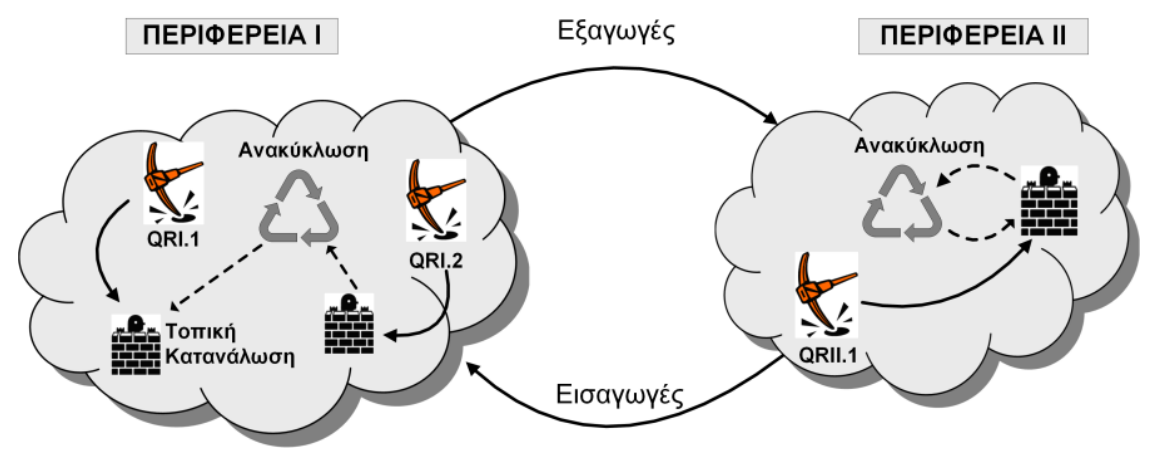

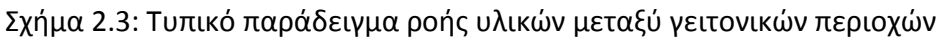

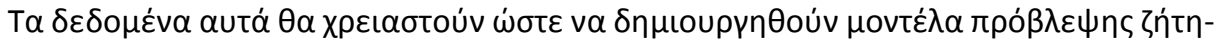

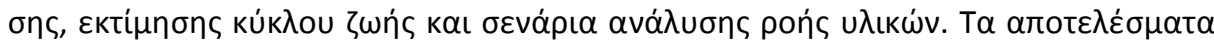

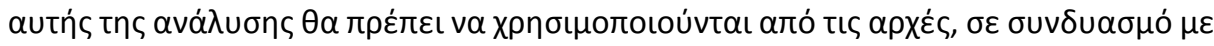

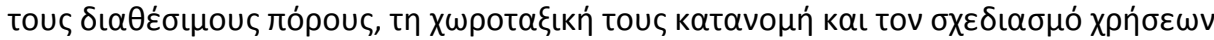

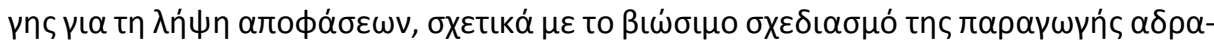

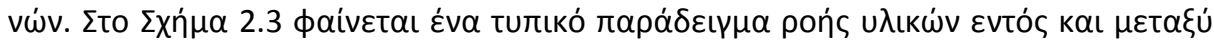

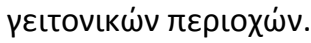

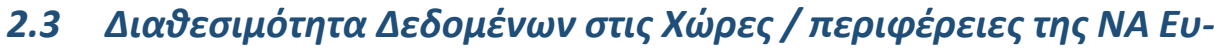

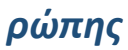

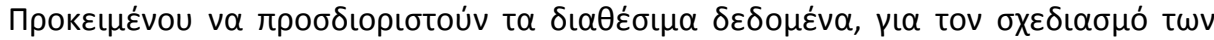

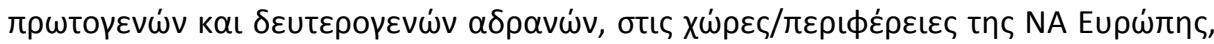

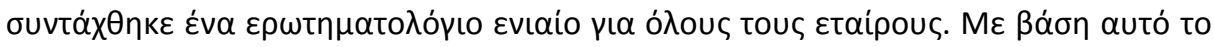

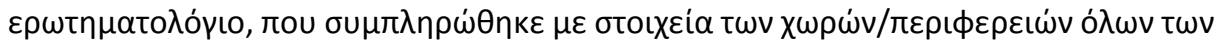

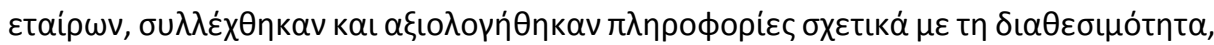

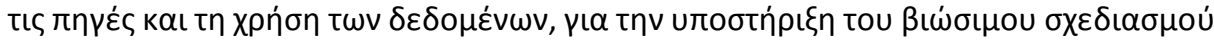

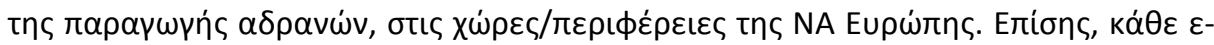

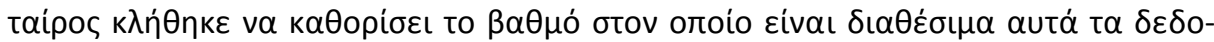

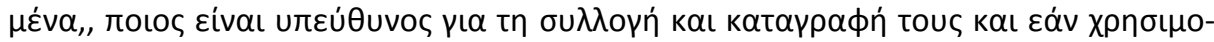

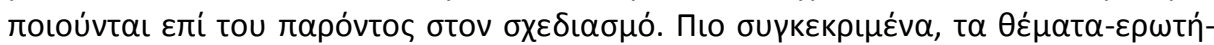

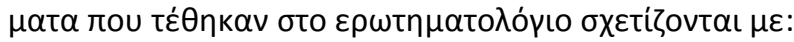

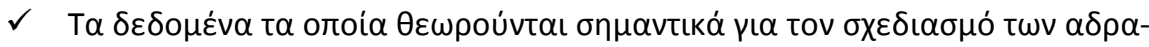
vúv.

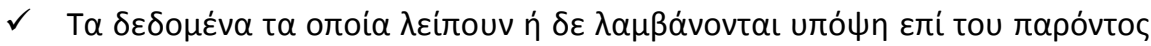

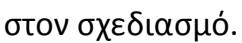




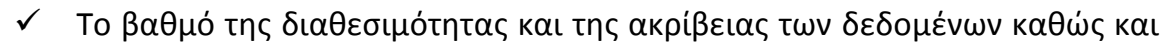

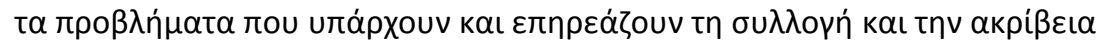
$\tau \omega v \delta \varepsilon \delta o \mu \varepsilon ́ v \omega v$.

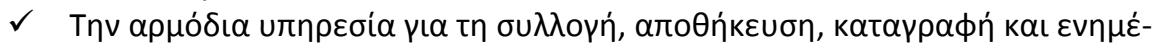
$\rho \omega \sigma \eta \tau \omega v \delta \varepsilon \delta \circ \mu \varepsilon \dot{v} \omega v$.

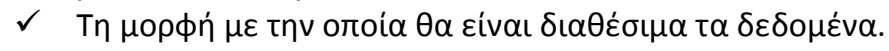

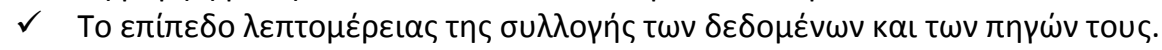

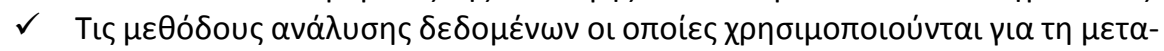

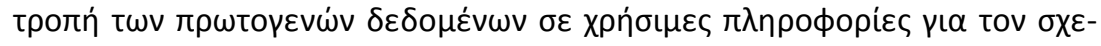

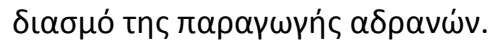

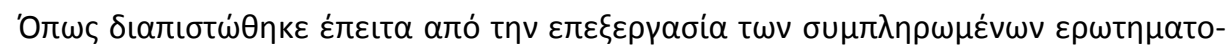

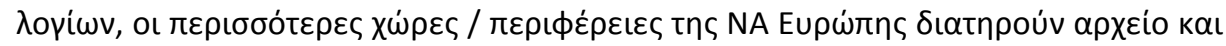

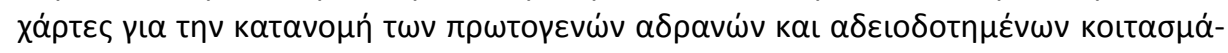

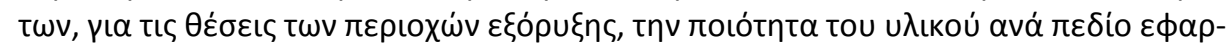

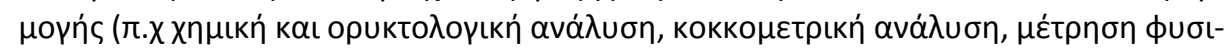

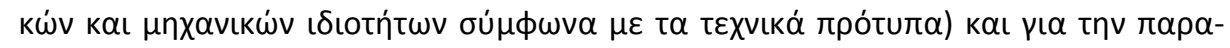

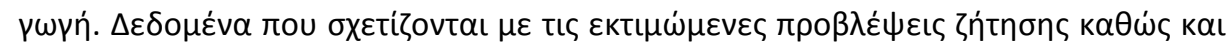

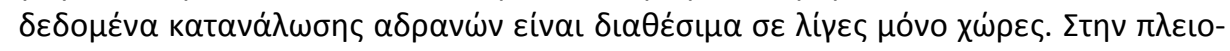

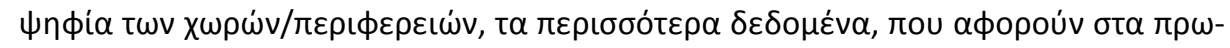

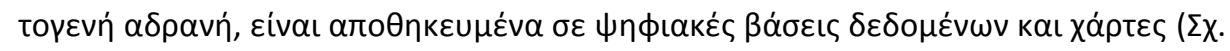
2.4).

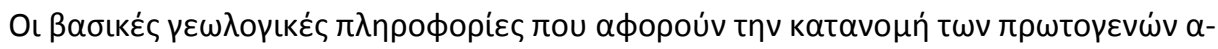

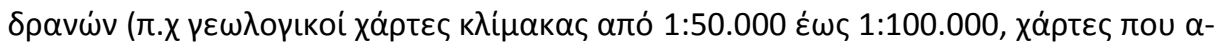

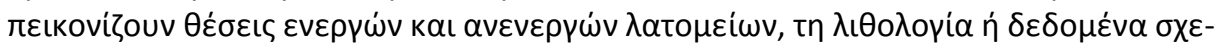

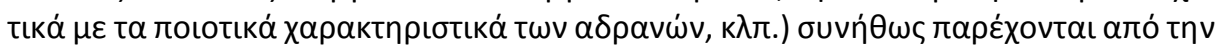

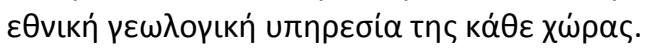

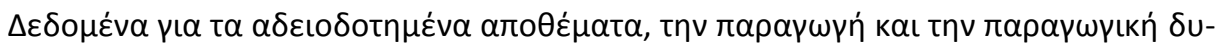

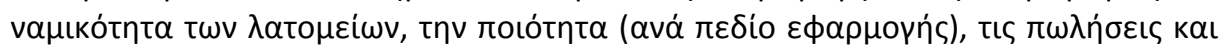

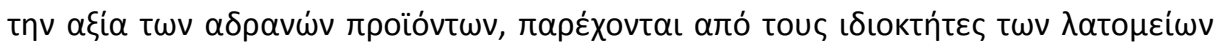

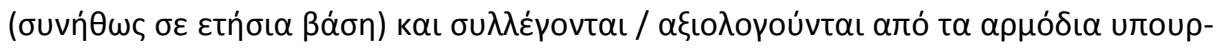

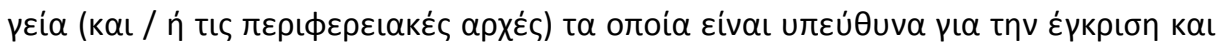

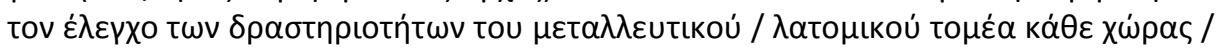

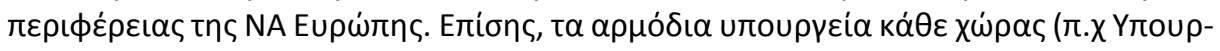

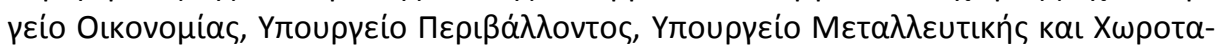

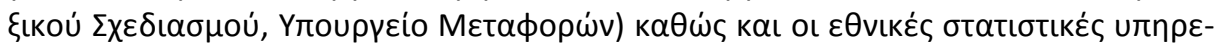

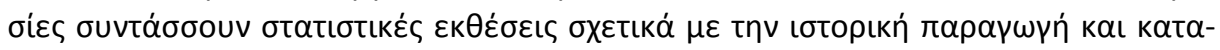

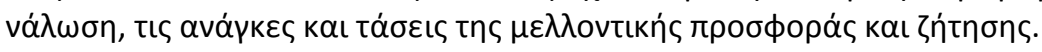




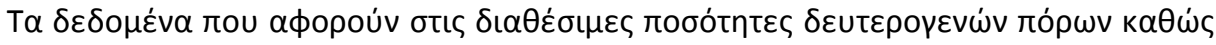

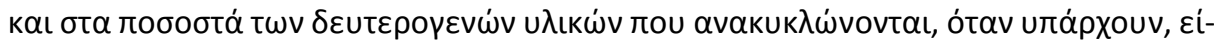

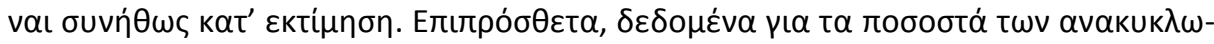

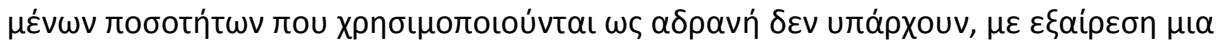

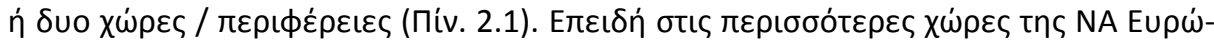

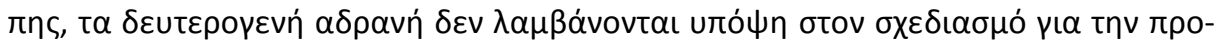

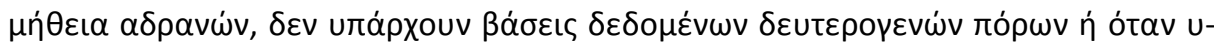

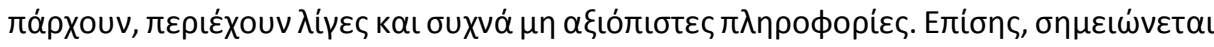

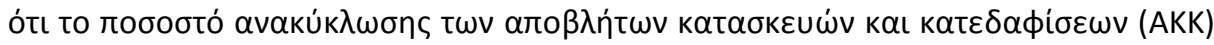

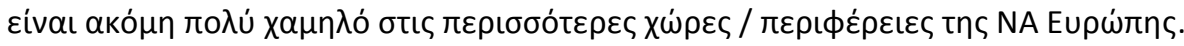

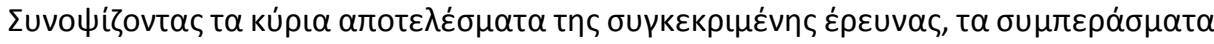

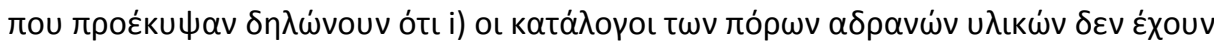

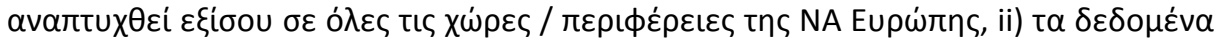

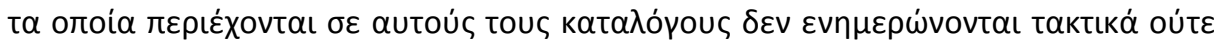

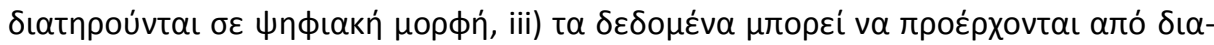

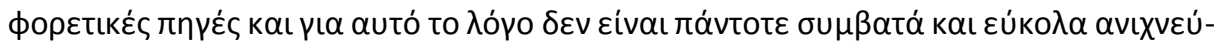

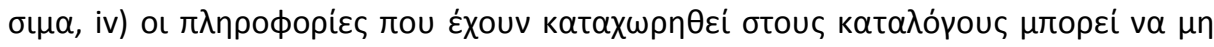

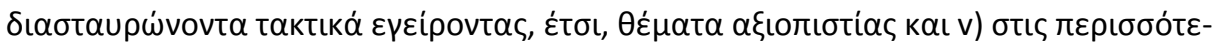

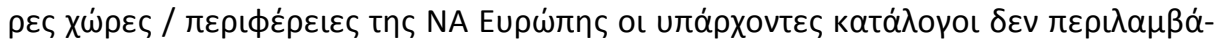

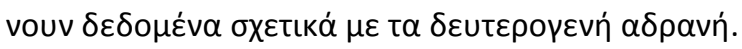

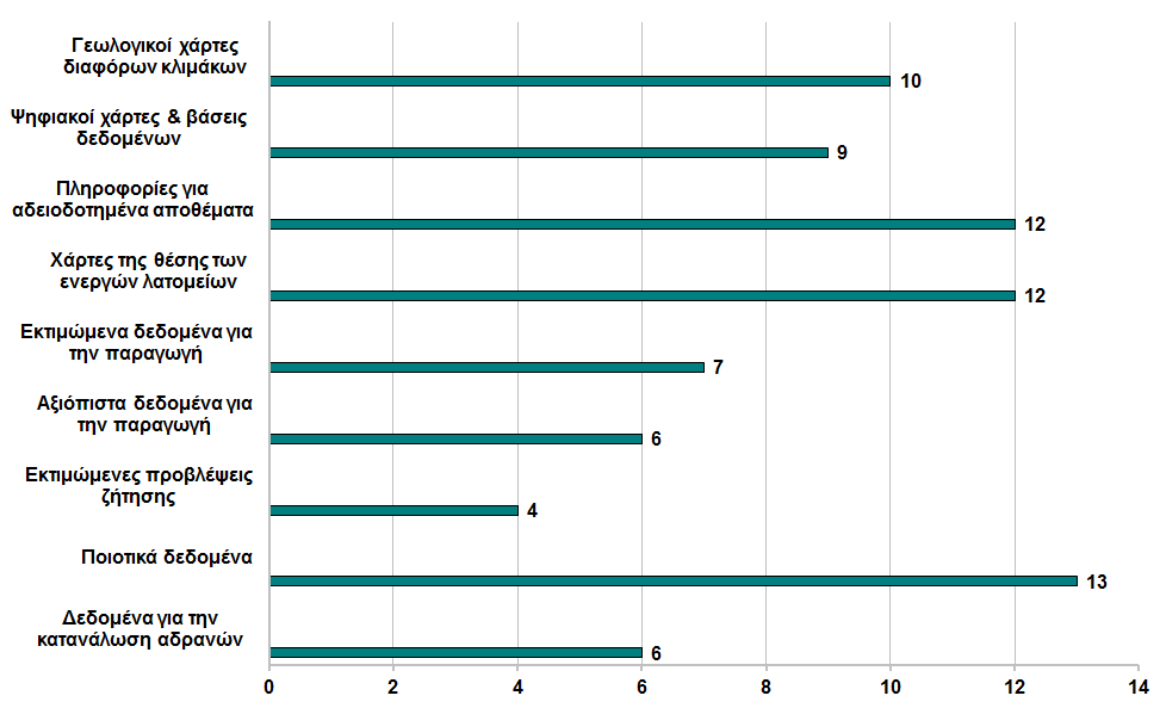

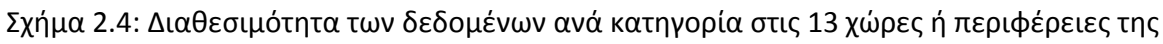
NA Eupẃrnৎ (SEE) (Hatzilazaridou, 2014) 


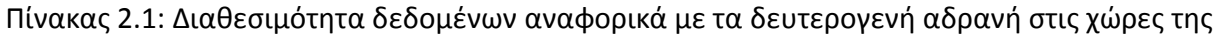
NA Eupúrns (Hatzilazaridou, 2014)

\begin{tabular}{|c|c|c|c|c|c|c|}
\hline \multirow[b]{2}{*}{ 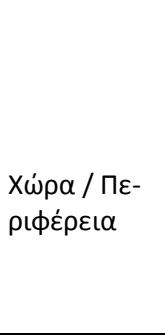 } & \multicolumn{4}{|c|}{ 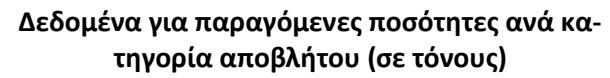 } & \multirow[b]{2}{*}{ 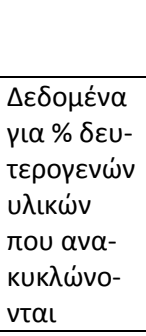 } & \multirow[b]{2}{*}{ 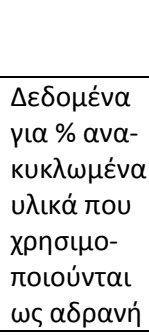 } \\
\hline & $\mathrm{R} 1\left(^{*}\right)$ & $\mathrm{R} 2\left(^{*}\right)$ & R3 $(*)$ & $\mathrm{R} 4\left(^{*}\right)$ & & \\
\hline$A \lambda \beta a v i \alpha$ & Ектінпоп & OXI & OXI & 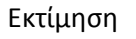 & OXI & OXI \\
\hline Aибтрі́ & 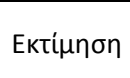 & $\mathrm{NAI}$ & OXI & $\mathrm{NAI}$ & $\begin{array}{l}\text { NAI (R2) } \\
\text { NAI (R4) }\end{array}$ & OXI \\
\hline 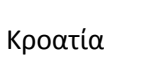 & 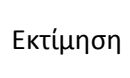 & $\begin{array}{l}\text { Eкrí- } \\
\mu \eta \sigma \eta\end{array}$ & $\begin{array}{l}\text { Ektí- } \\
\mu \eta \sigma \eta\end{array}$ & Екті $\mu \eta \sigma \eta$ & OXI & OXI \\
\hline 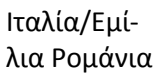 & NAI & NAI & OXI & NAI & NAl & OXI \\
\hline$E \lambda \lambda \alpha \dot{\alpha} \delta \alpha$ & OXI & $\begin{array}{l}\text { Eкti- } \\
\mu \eta \sigma \eta\end{array}$ & OXI & Екті́ $\mu \eta \sigma \eta$ & OXI & OXI \\
\hline 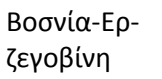 & OXI & OXI & OXI & OXI & OXI & OXI \\
\hline Oupvapia & Ектінпоп & $\begin{array}{l}\text { Eкrí- } \\
\mu \eta \sigma \eta\end{array}$ & OXI & Екті́рпоп & NAI & OXI \\
\hline $\begin{array}{l}\text { Maupoßoú- } \\
\text { vio }\end{array}$ & Eктínбо & OXI & OXI & Екті́цпоп & OXI & OXI \\
\hline Poupavía & Ектінпоп & OXI & OXI & Екті́ $\mu \eta \sigma \eta$ & OXI & OXI \\
\hline$\sum \varepsilon \rho \beta i \alpha$ & Eктínбп & OXI & OXI & Екті́ $\mu \eta \sigma \eta$ & $\begin{array}{l}\text { NAI (R1) } \\
\text { OXI (R4) }\end{array}$ & $\begin{array}{l}\text { NAI (R1) } \\
\text { OXI (R4) }\end{array}$ \\
\hline$\Sigma \lambda \circ \beta \alpha$ кі $\alpha$ & Eктínоn & $\begin{array}{l}\text { Eкti- } \\
\mu \eta \sigma \eta\end{array}$ & OXI & Екті́цпоп & $\begin{array}{l}\text { OXI (R1) } \\
\text { NAI (R2) } \\
\text { NAI ( R4) }\end{array}$ & OXI \\
\hline$\Sigma \lambda \circ \beta \varepsilon v i \alpha$ & Ектінпоп & $\mathrm{NAI}$ & OXI & Екті́цпоп & $\begin{array}{l}\text { NAI ( R1) } \\
\text { NAI ( R2) } \\
\text { OXI (R4) }\end{array}$ & OXI \\
\hline 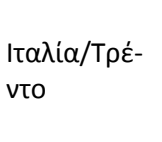 & Ектінпоп & NAI & $\begin{array}{l}\text { Ekti- } \\
\mu \eta \sigma \eta\end{array}$ & NAI & $\begin{array}{l}\text { OXI (R1) } \\
\text { NAI (R2) } \\
\text { OXI (R3) } \\
\text { NAI (R4) }\end{array}$ & NAI (R2) \\
\hline
\end{tabular}

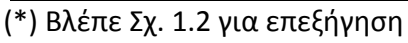




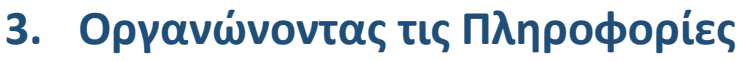

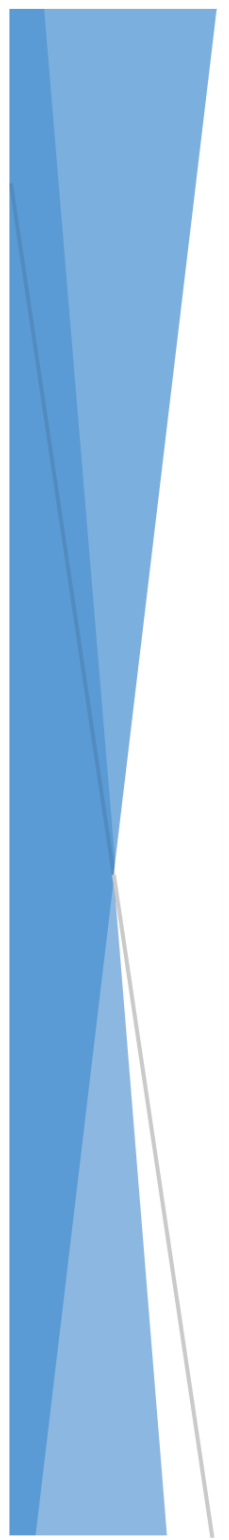

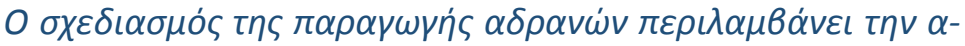

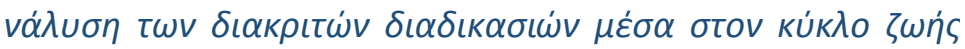

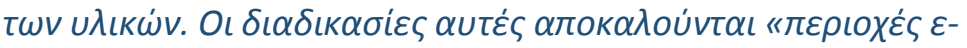

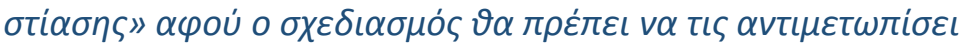

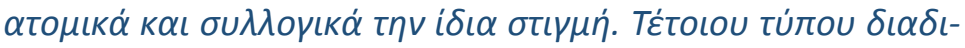

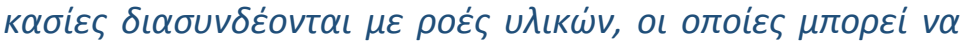

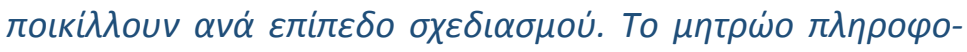

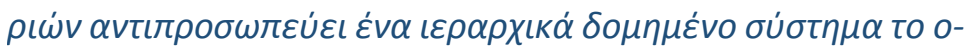

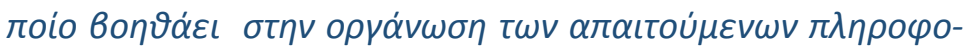

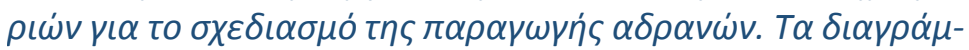

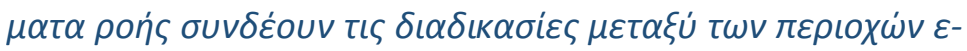

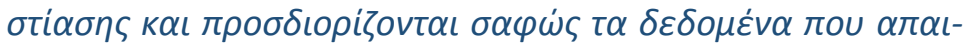

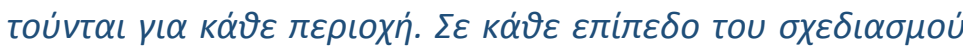

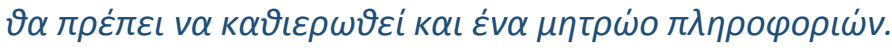




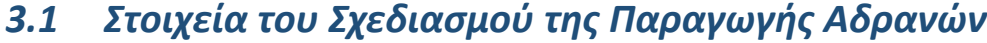

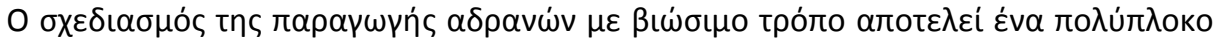

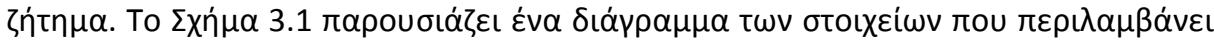

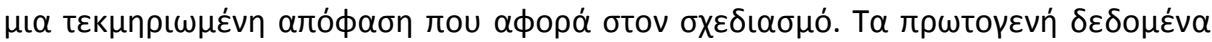

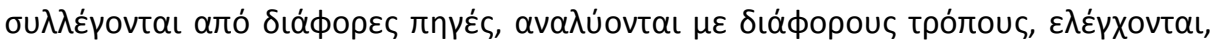

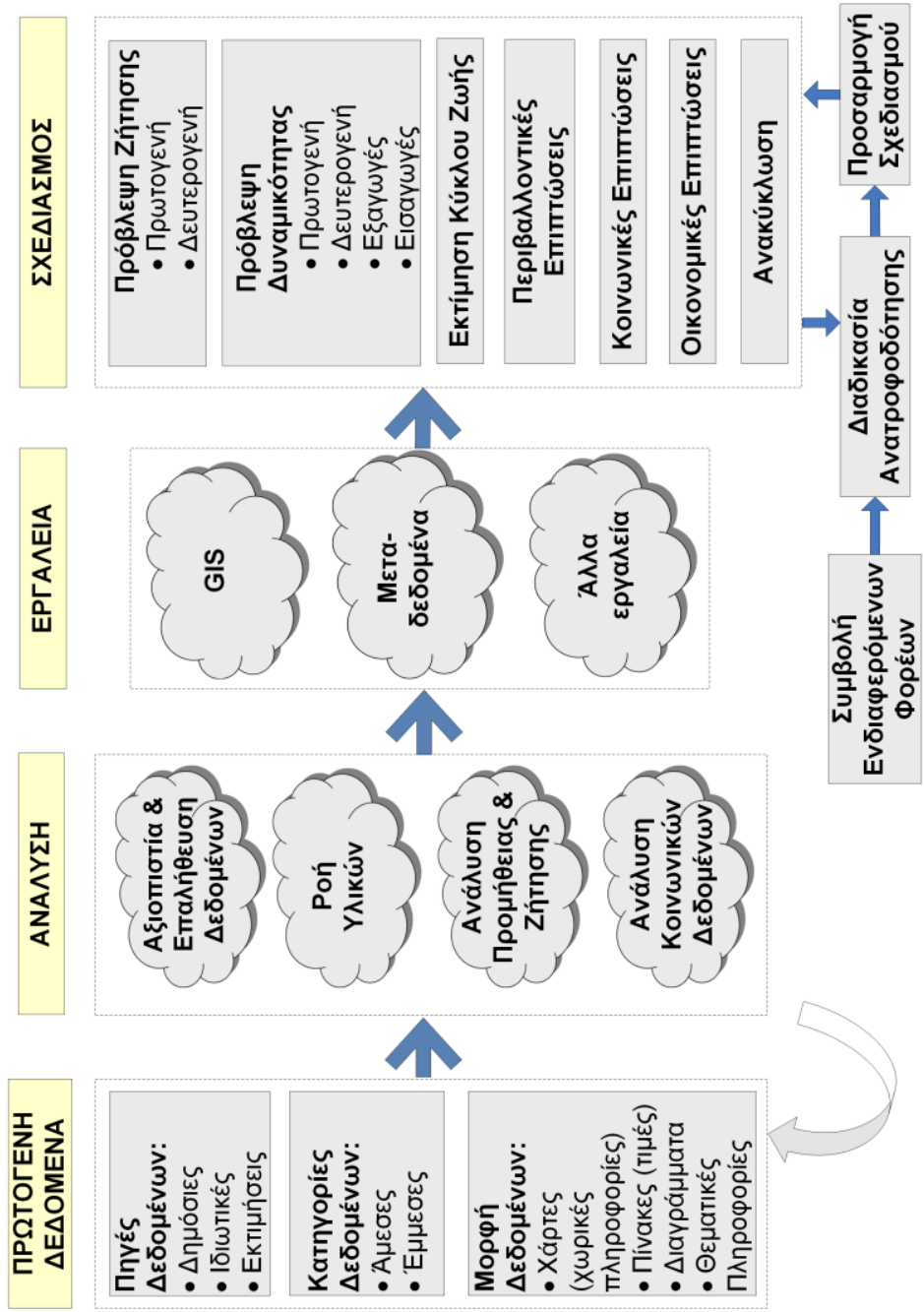

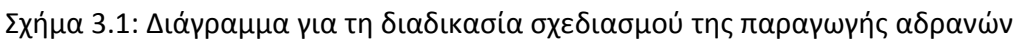




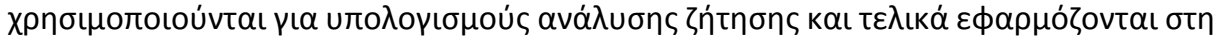

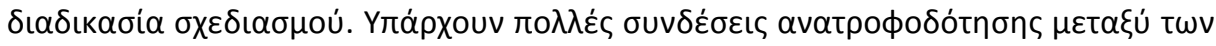

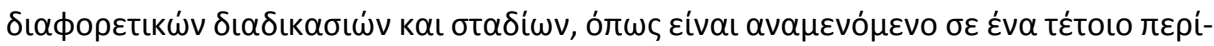

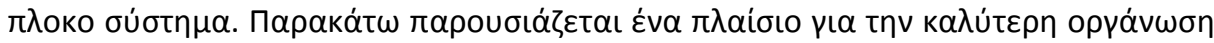

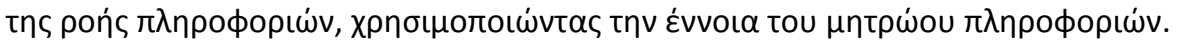

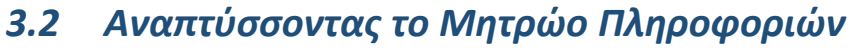

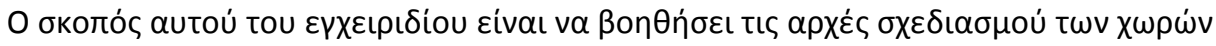

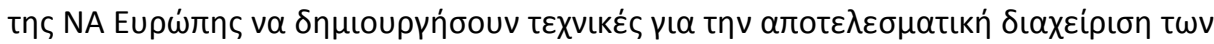

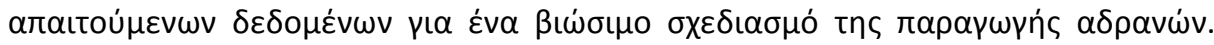

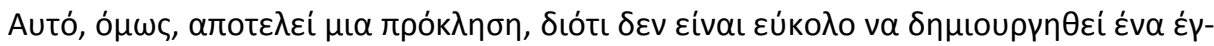

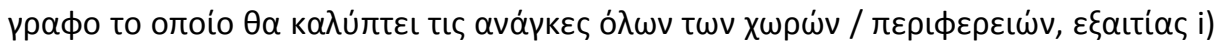

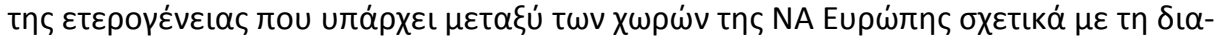

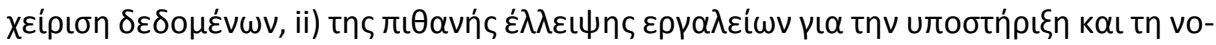

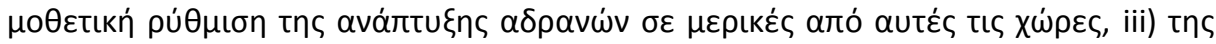

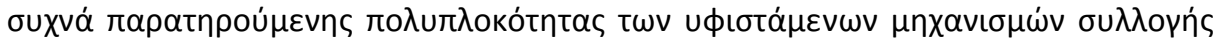

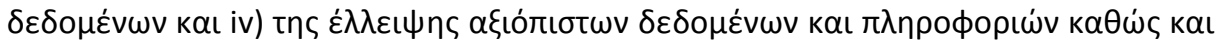

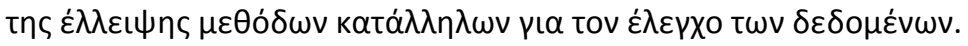

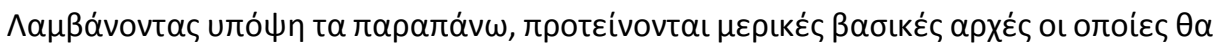

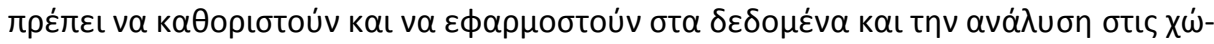

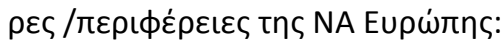

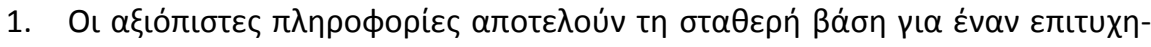
$\mu \varepsilon \dot{v}$ o $\chi \varepsilon \delta\llcorner\alpha \sigma \mu o ́$.

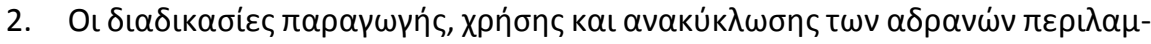

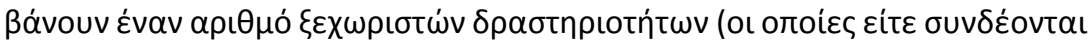

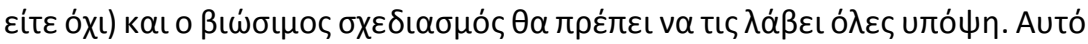

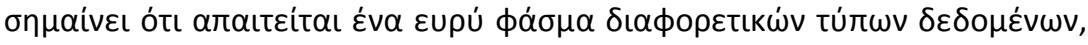

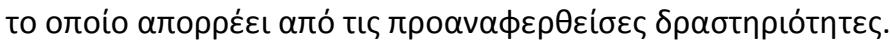

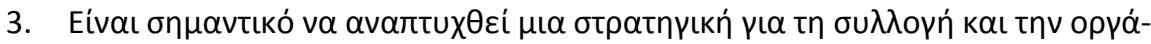

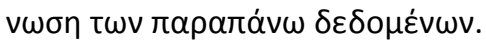

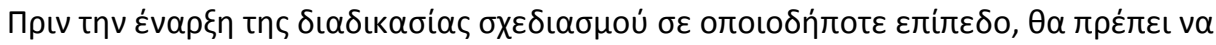

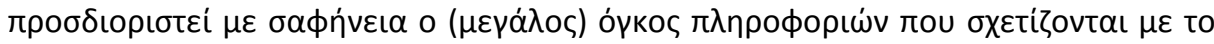
биүкєкрцив́

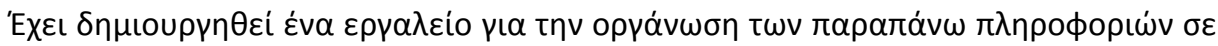

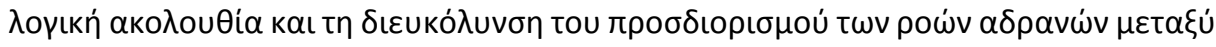




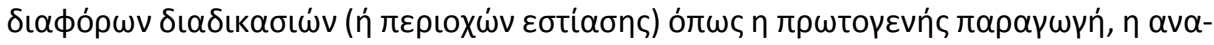

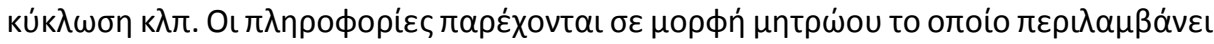

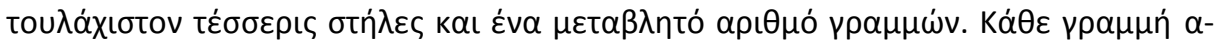

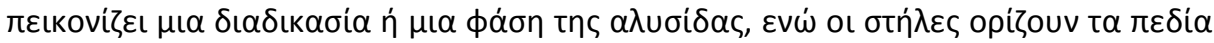

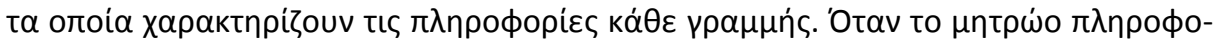

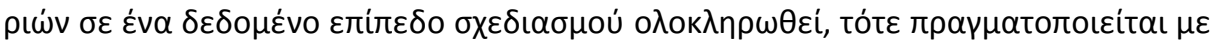

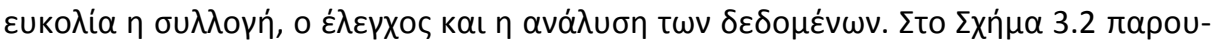

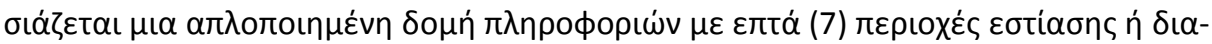

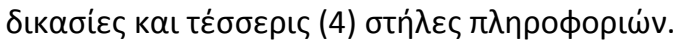

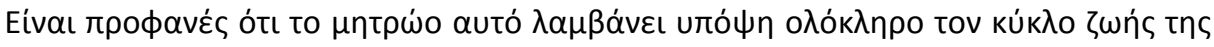

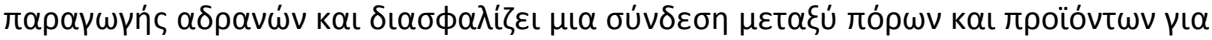

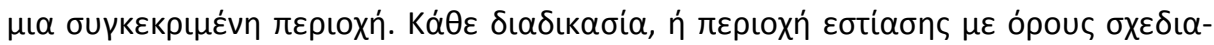

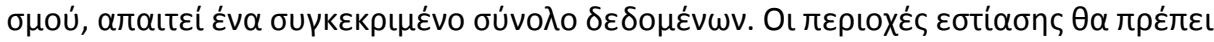

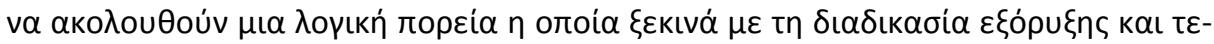

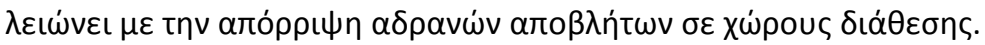

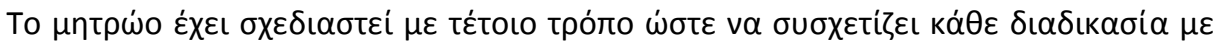

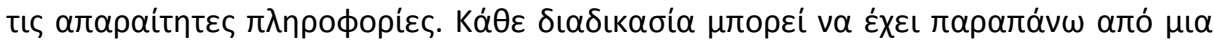

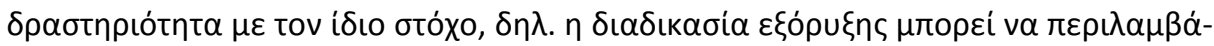

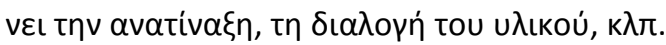

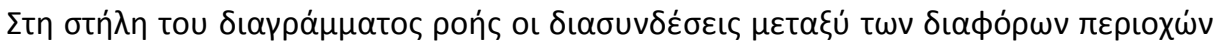

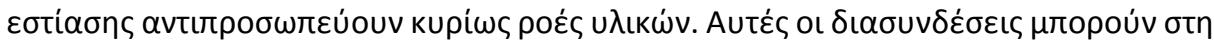

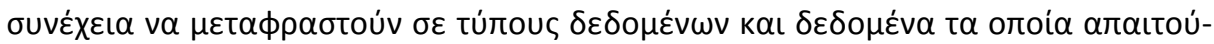

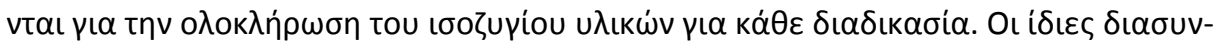

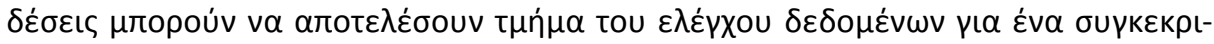

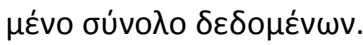

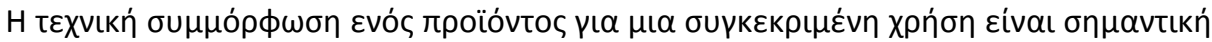

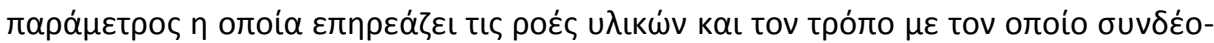

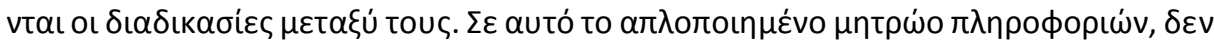

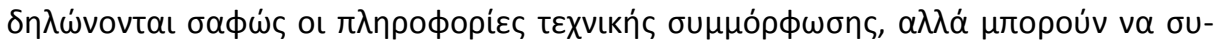

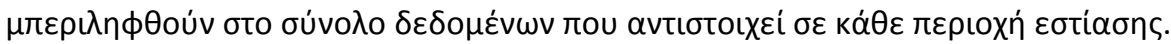

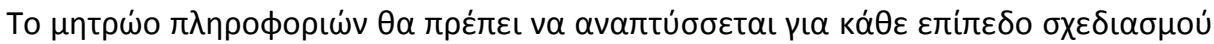

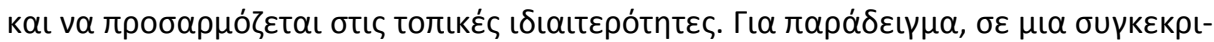

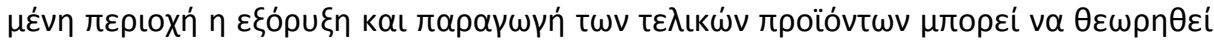

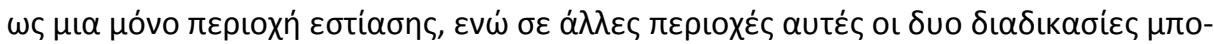

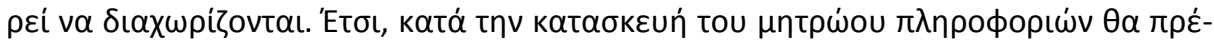

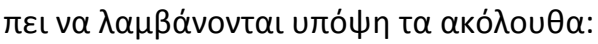




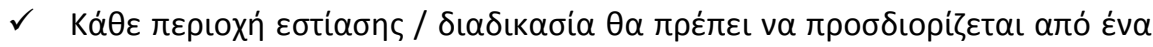
биүкєкрци́vo бтóxo.

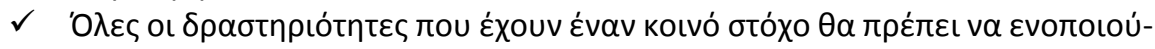

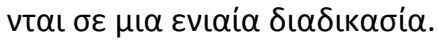

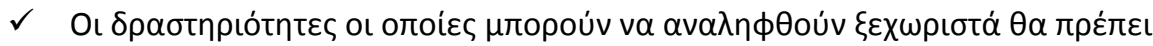

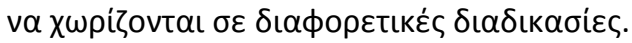

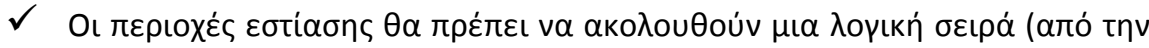

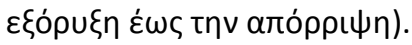

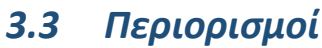

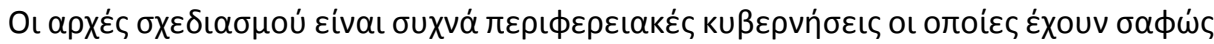

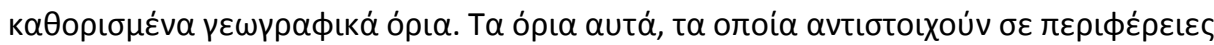

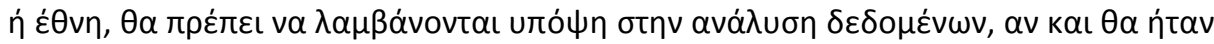

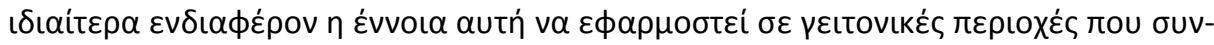

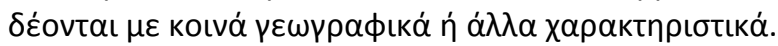

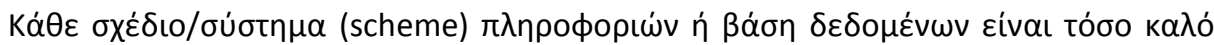

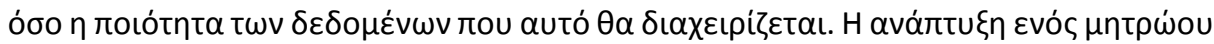

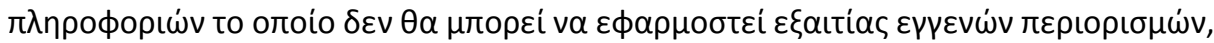

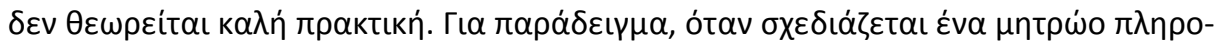

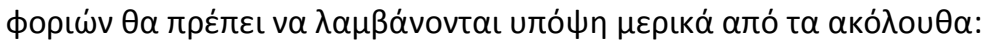

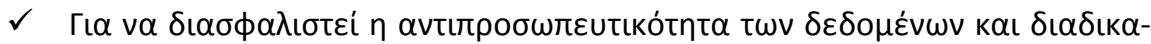

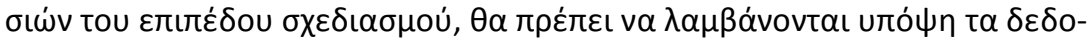

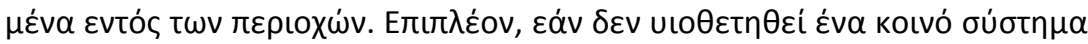

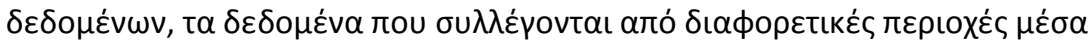

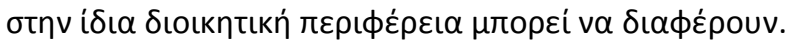

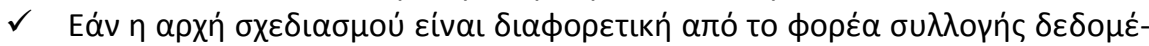

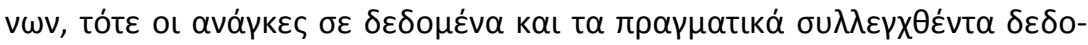

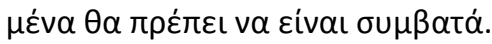

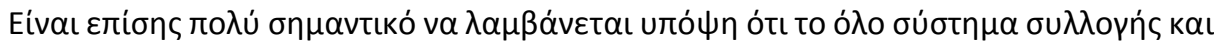

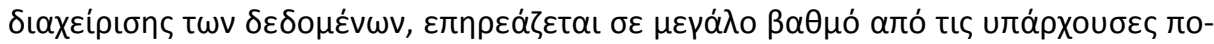

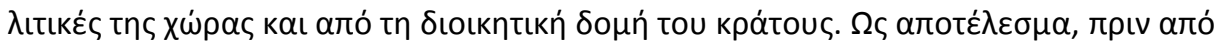

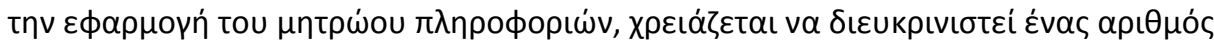

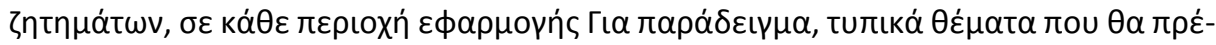

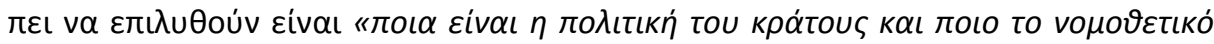

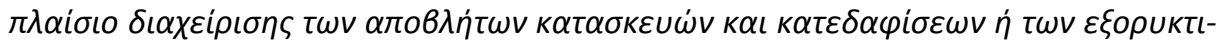
$\kappa \dot{v} v \alpha \pi \circ \beta \lambda \dot{\tau} \tau \omega v »$. 


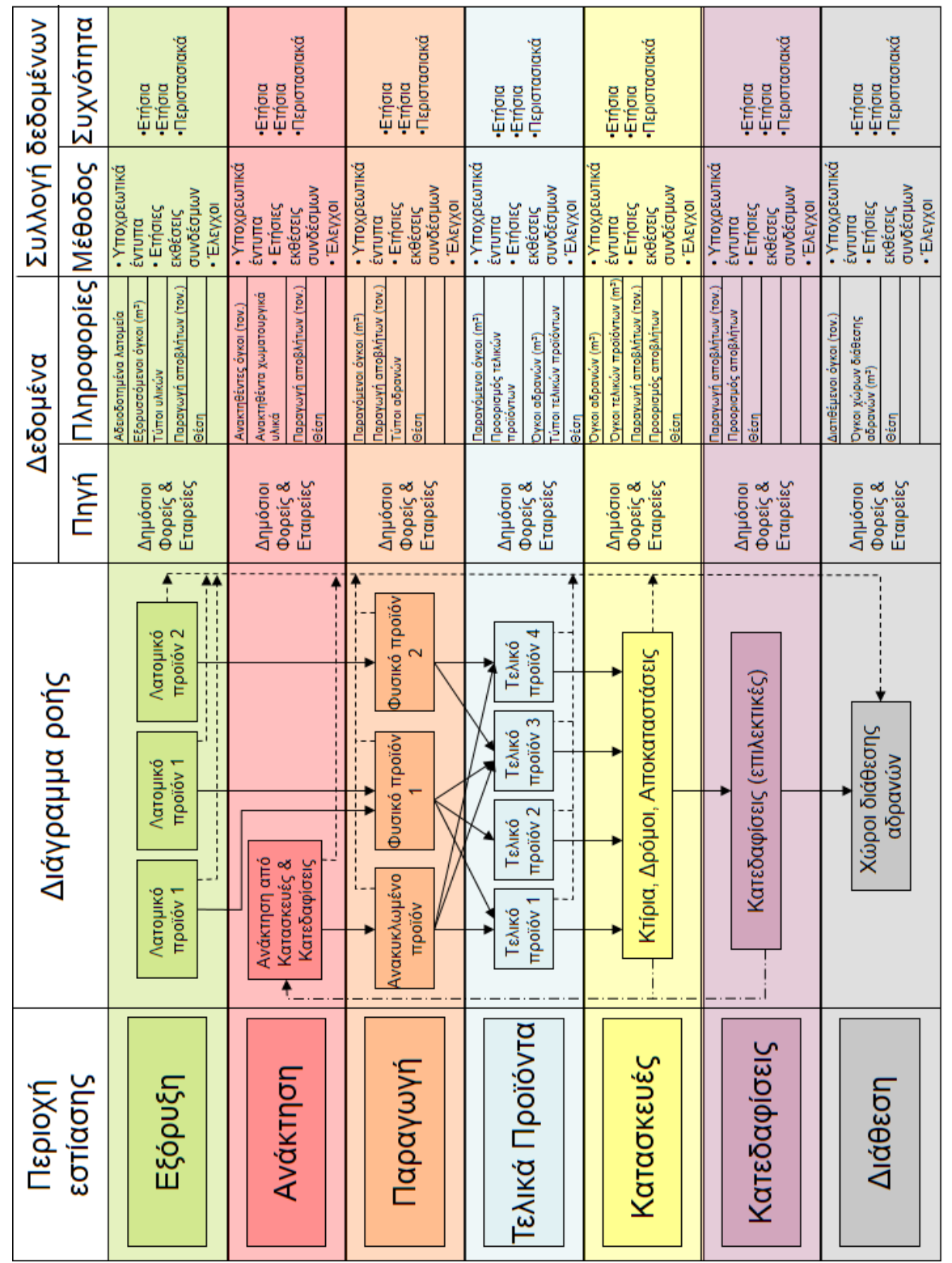

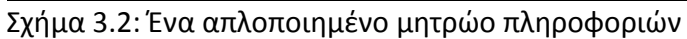




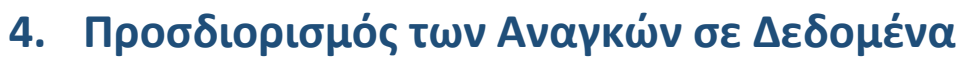

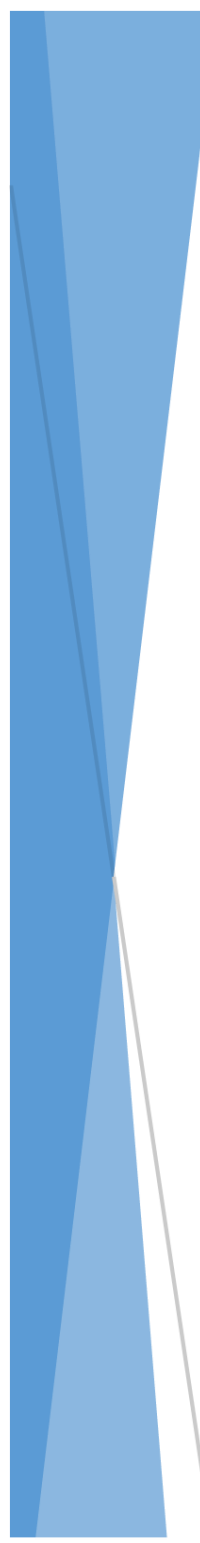

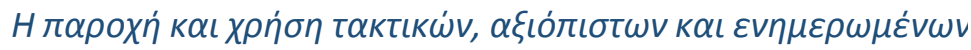

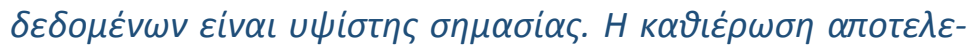

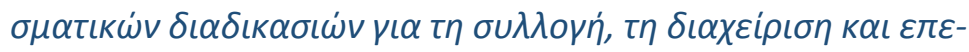

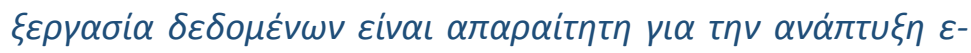

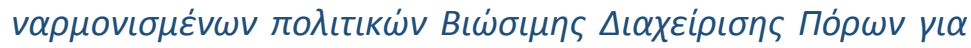

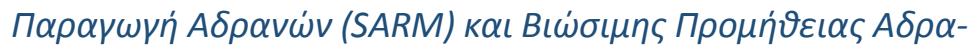

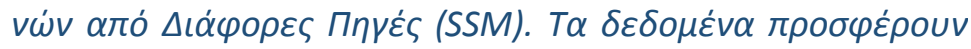

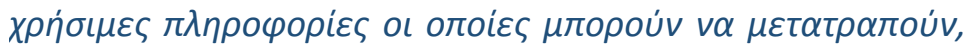

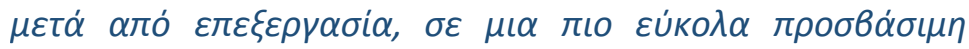

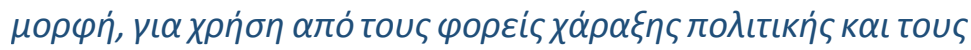

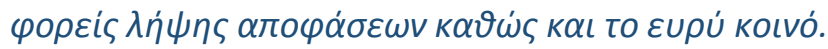

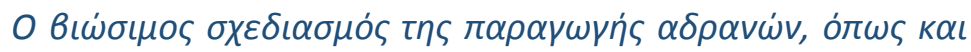

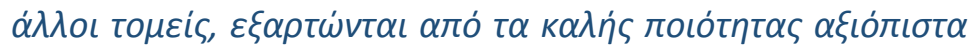

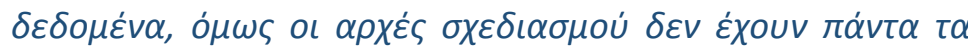

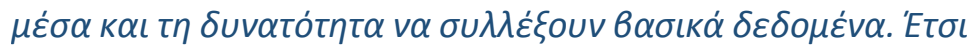

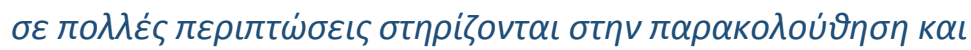

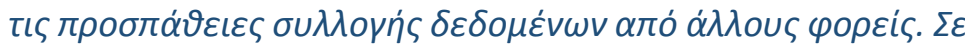

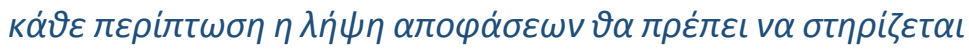

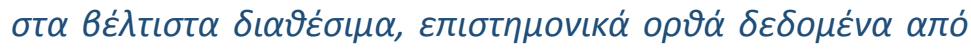

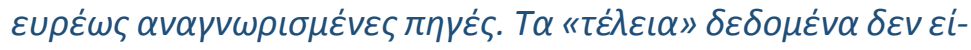

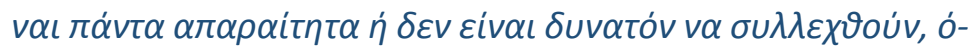

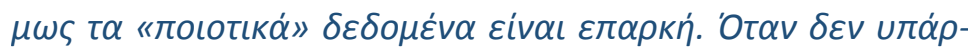

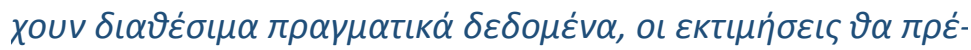

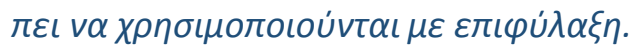




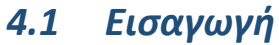

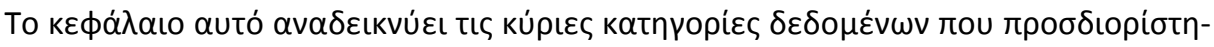

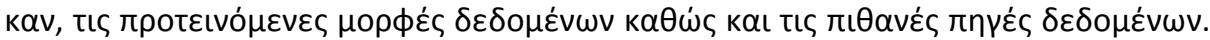

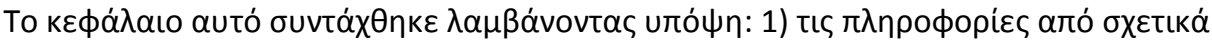

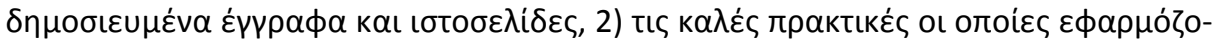

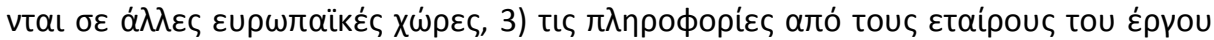

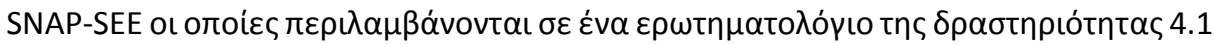

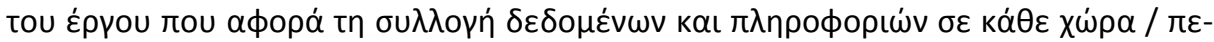

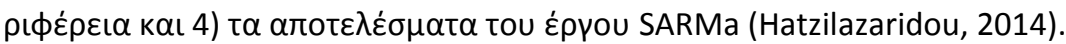

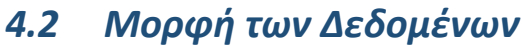

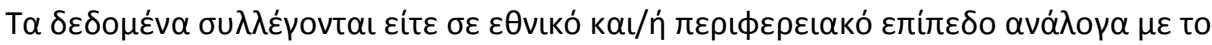

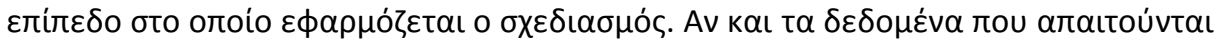

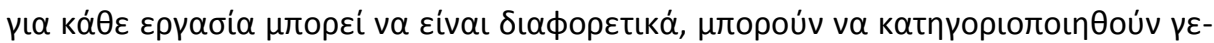

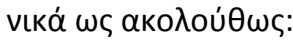

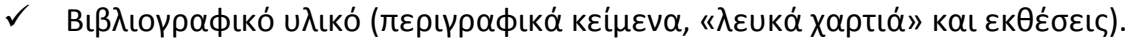

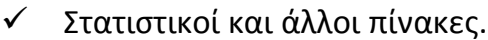

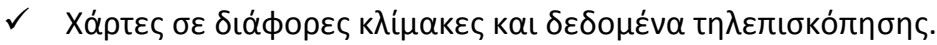

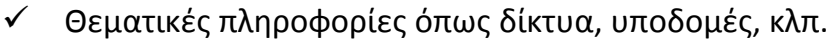

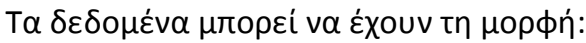

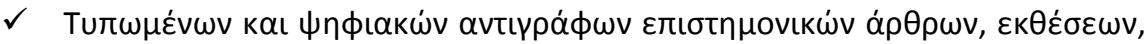

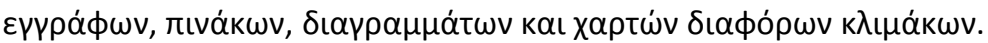

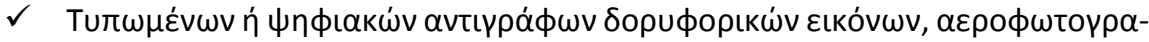

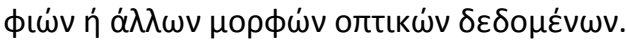

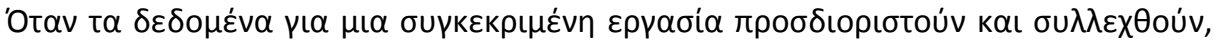

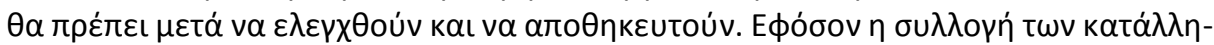

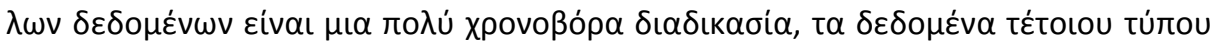

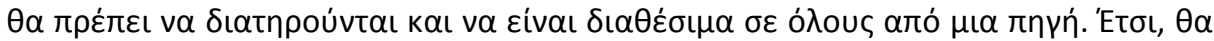

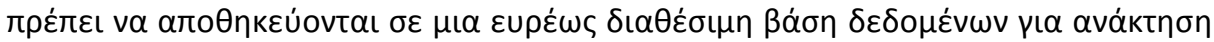

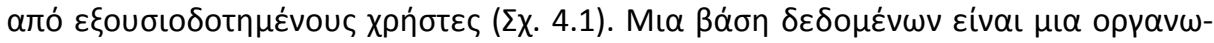

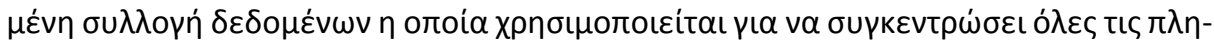

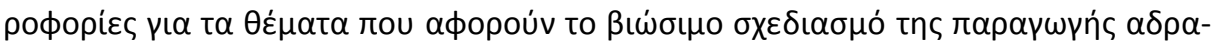

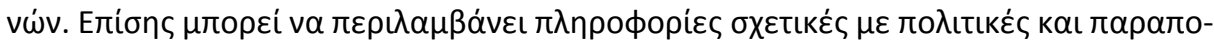
$\mu \pi \varepsilon \dot{~} \sigma \varepsilon \alpha \dot{\alpha} \lambda \lambda \varepsilon \varsigma \pi \eta \gamma \varepsilon \dot{\varepsilon} \delta \varepsilon \delta \circ \mu \varepsilon \dot{v} \omega \mathrm{V}$. 


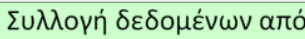

$\delta ı \alpha ́ \phi о \rho \varepsilon \varsigma ~ \pi \eta ү \varepsilon ́ \varsigma, ~ \sigma \varepsilon$

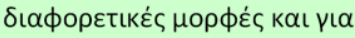

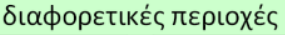

$E \lambda \varepsilon \gamma \chi \propto \varsigma \varsigma \sigma \phi \alpha \lambda \mu \alpha \dot{\tau} \tau \omega V$

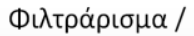

$\pi \rho \circ \pi \varepsilon \xi \varepsilon \rho \gamma \alpha \sigma i \alpha$

$\delta \varepsilon \delta \circ \mu \varepsilon \dot{v} \omega \mathrm{V}$

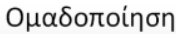

$\delta \varepsilon \delta \circ \mu \varepsilon \dot{v} \omega v$

Пробарноүи́

$\delta \varepsilon \delta \circ \mu \varepsilon \dot{v} \omega v$ үı $\alpha$

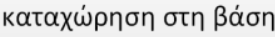

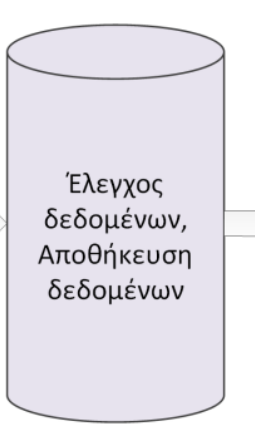

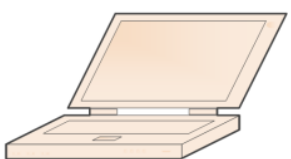

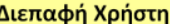

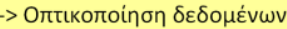

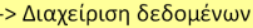

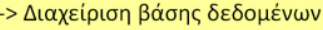

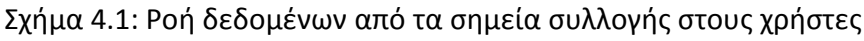

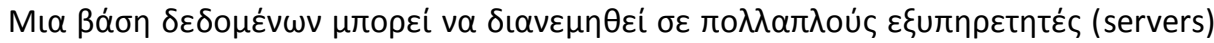

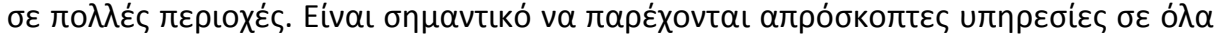

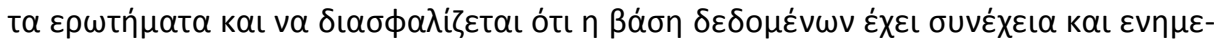

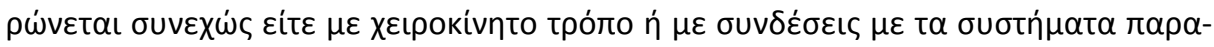

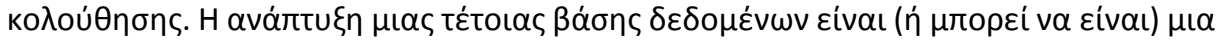

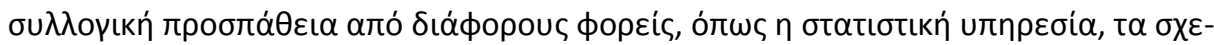

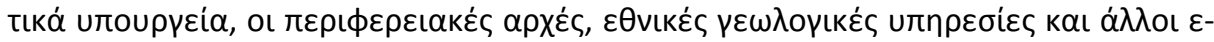

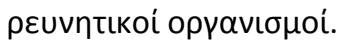

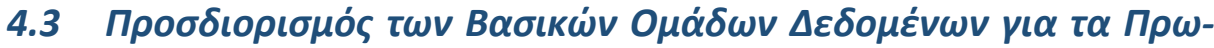

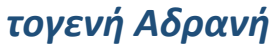

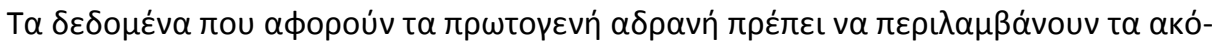
$\lambda \circ \theta_{\alpha}$ :

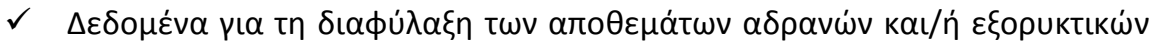

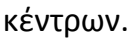

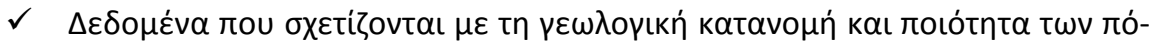
$\rho \omega v$.

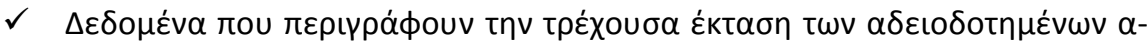

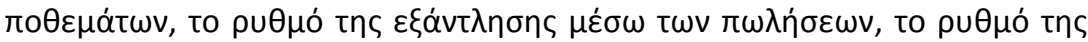




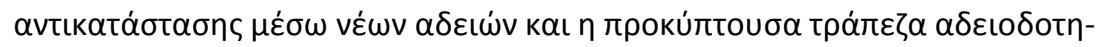
$\mu \varepsilon \dot{v} \omega v$ a $\pi 0 \theta \varepsilon \mu \alpha \dot{\tau} \tau \omega v$ (landbanks).

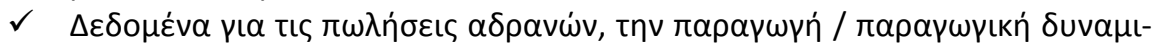

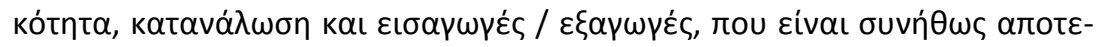

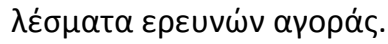

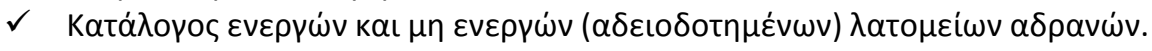

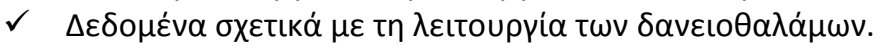

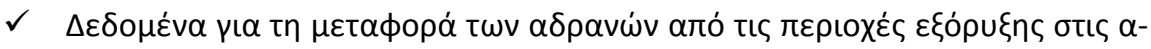

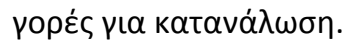

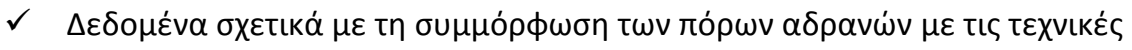
$\pi \rho \circ \delta\llcorner\alpha p \rho \alpha \phi \varepsilon \dot{~}$

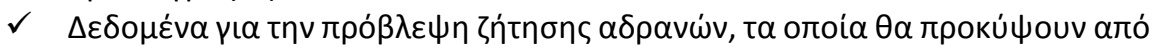

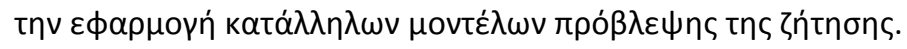

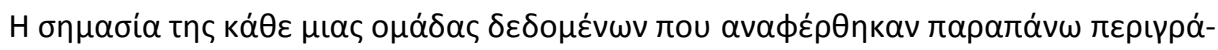

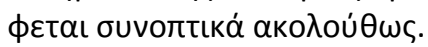

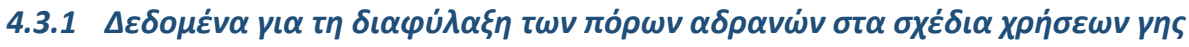

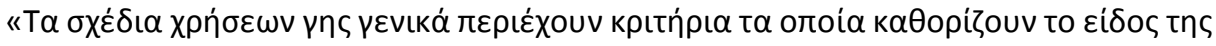

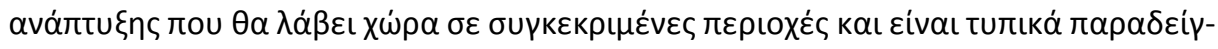

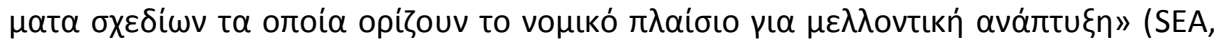
2001).

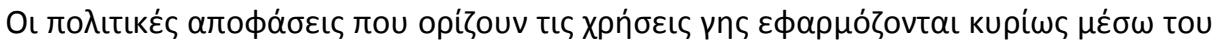

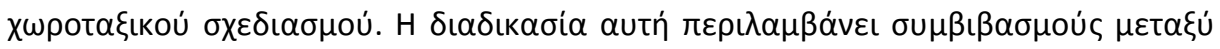

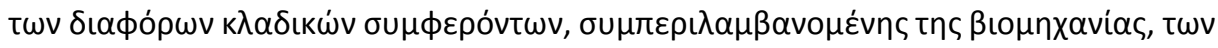

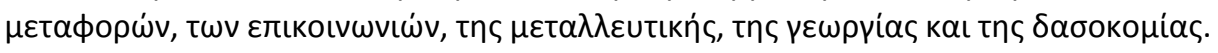

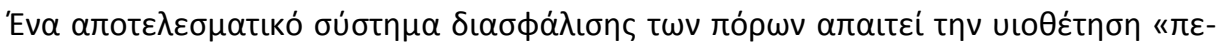

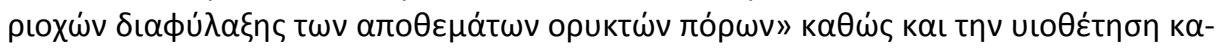

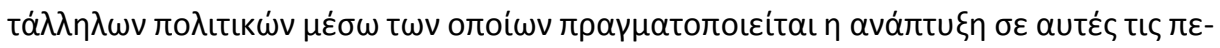

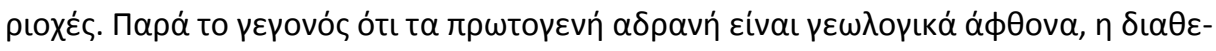

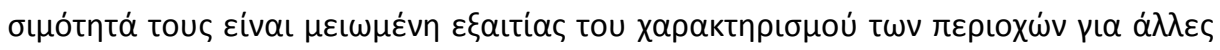

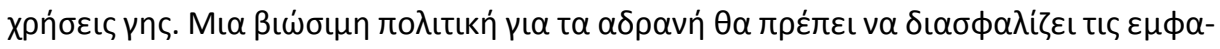

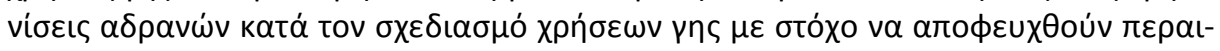

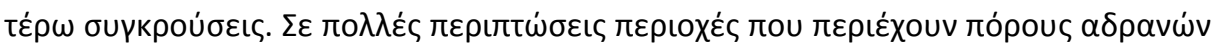

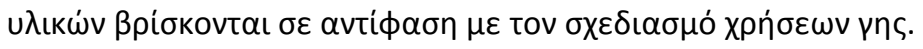




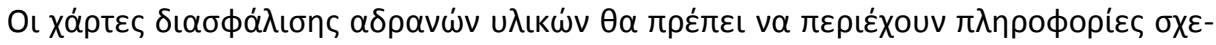

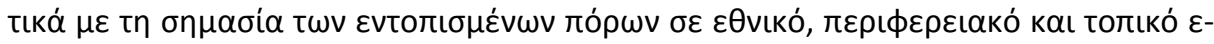

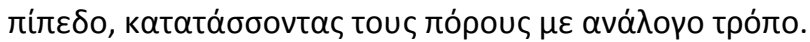

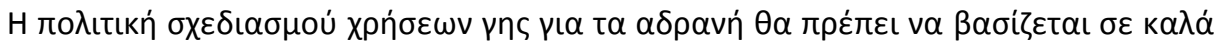

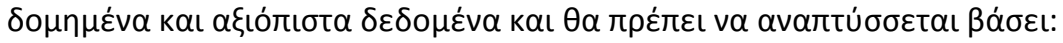

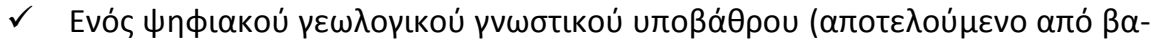

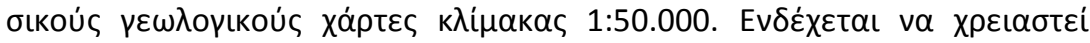

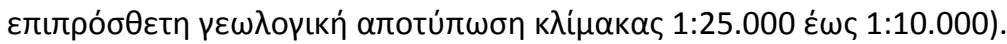

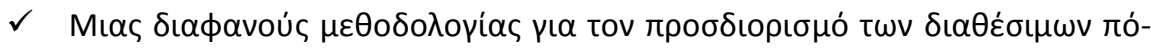

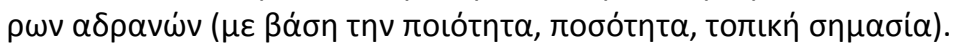

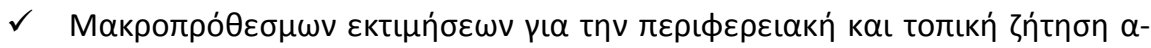

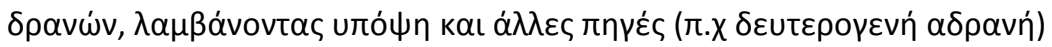

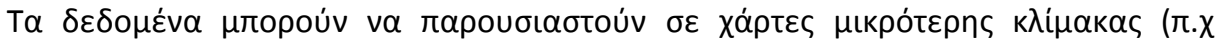

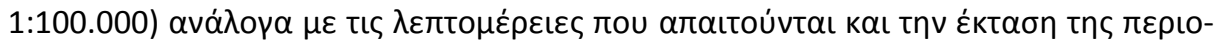

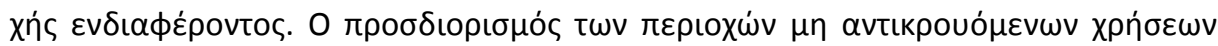

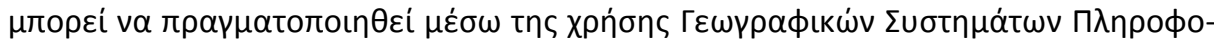

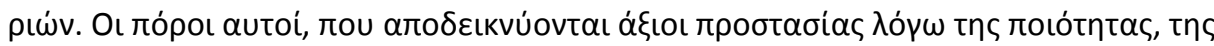

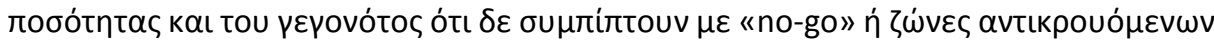

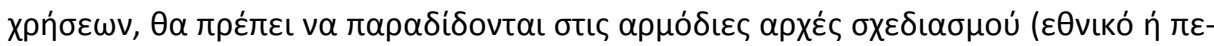

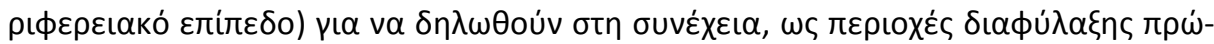

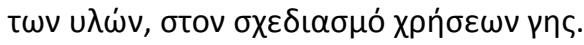

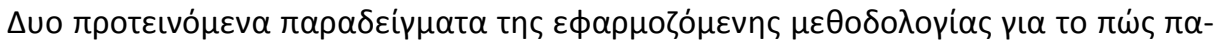

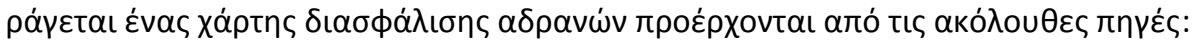

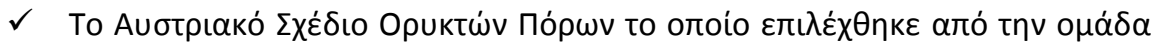

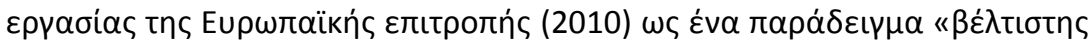

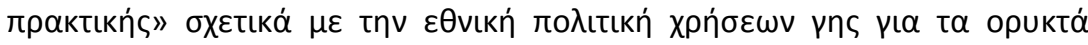
(Austria, 2014).

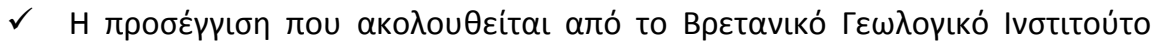
(BGS, 2012).

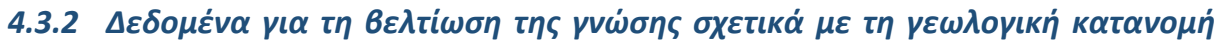

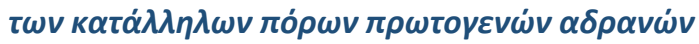

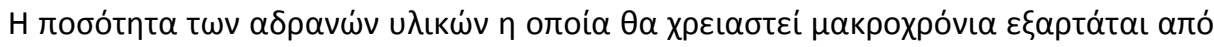

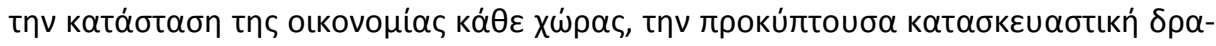

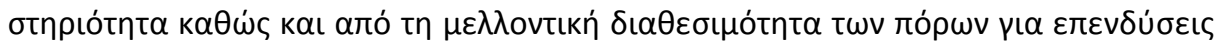

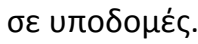




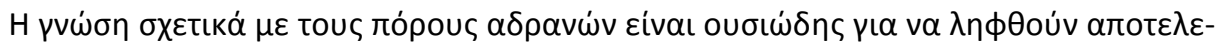

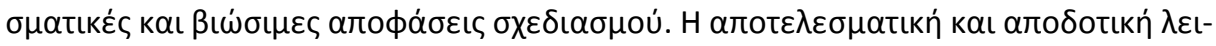

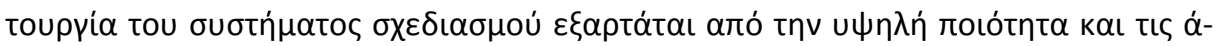

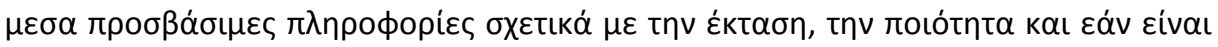

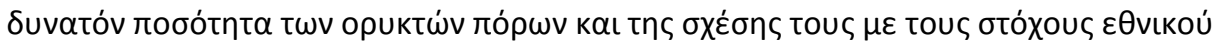

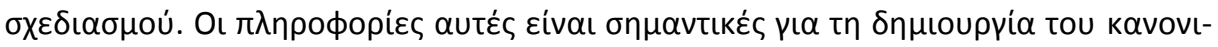

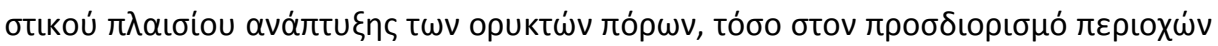

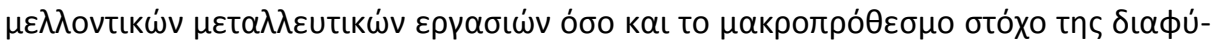

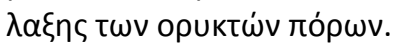

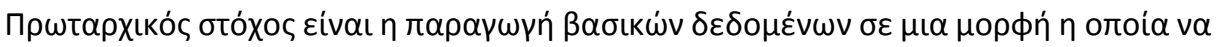

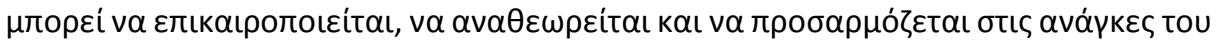

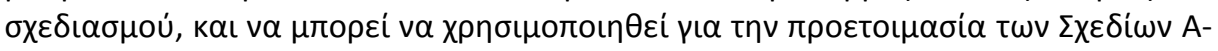

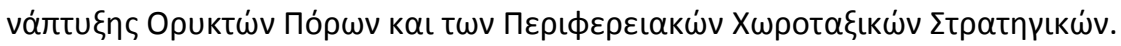

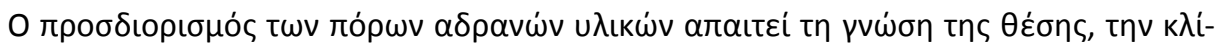

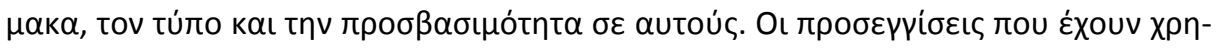

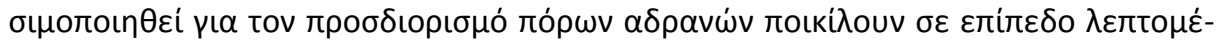

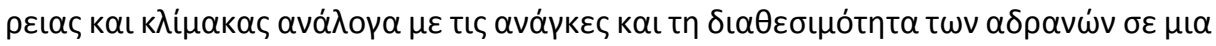

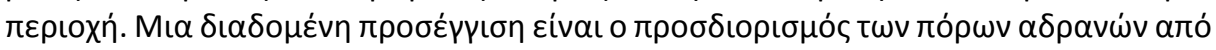

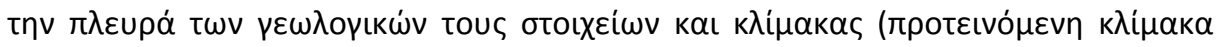

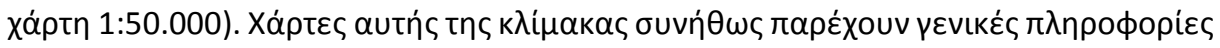

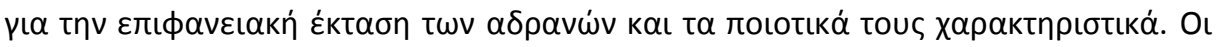

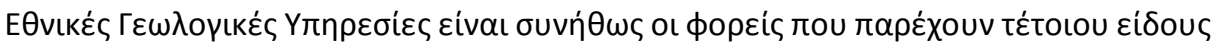
$\delta \varepsilon \delta \circ \mu \varepsilon \dot{v} \alpha$.

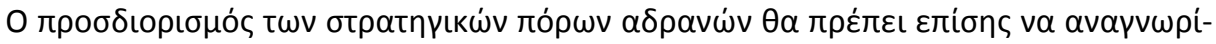

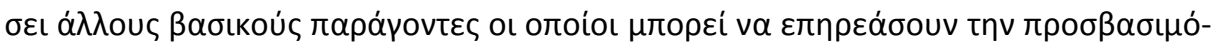

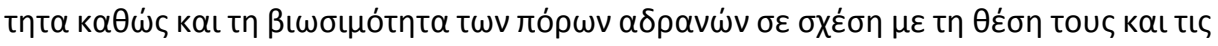

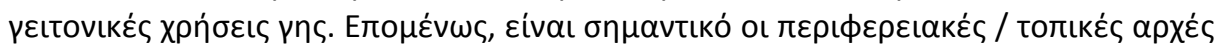

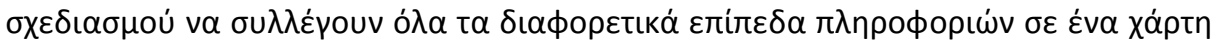

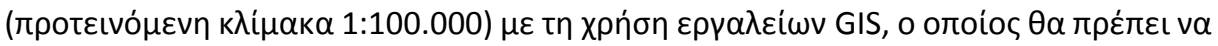

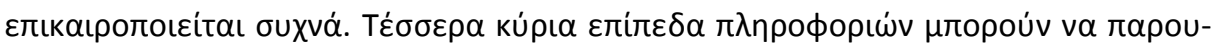

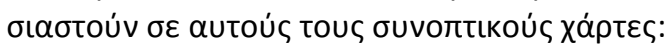

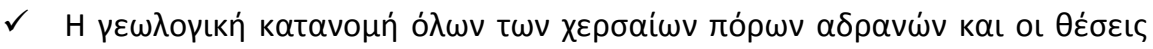

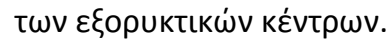

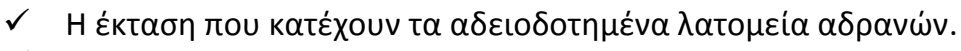

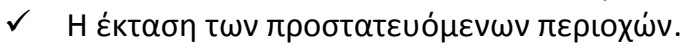




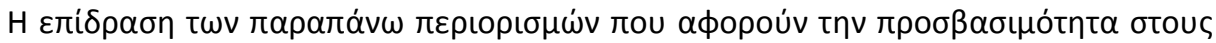

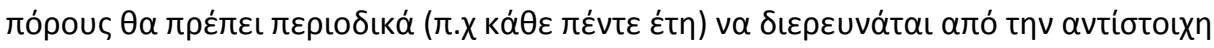

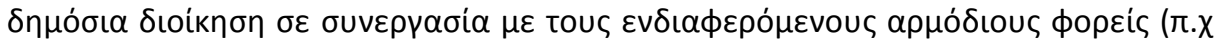

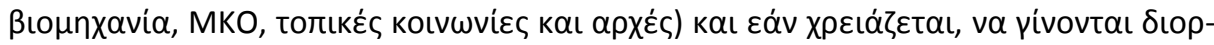

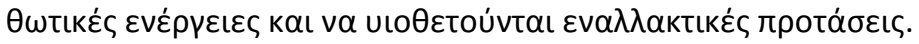

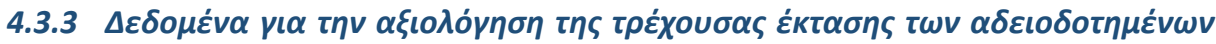

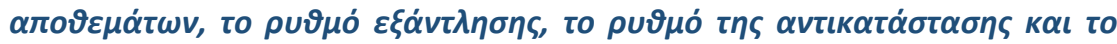

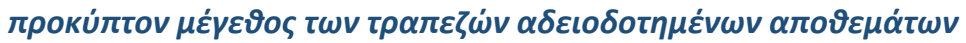

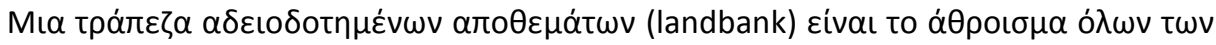

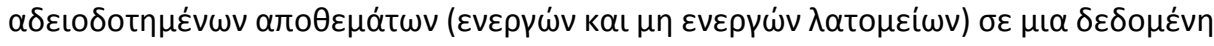

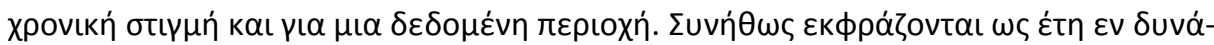

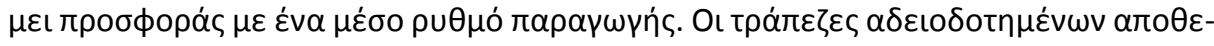

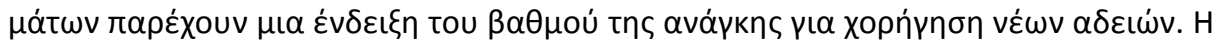

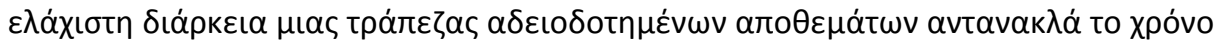

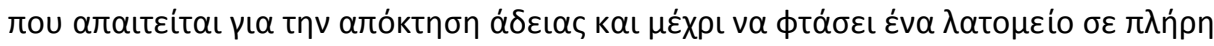

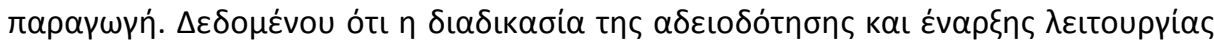

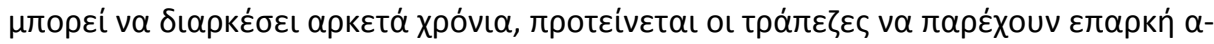

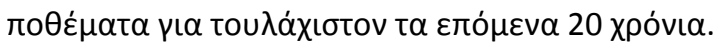

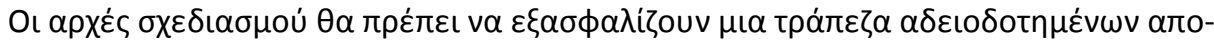

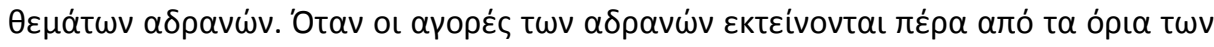

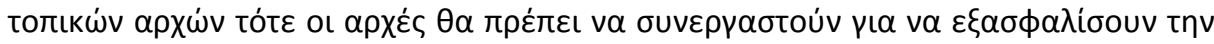

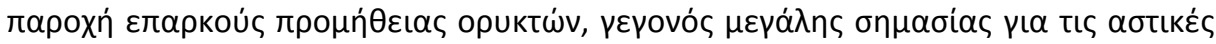

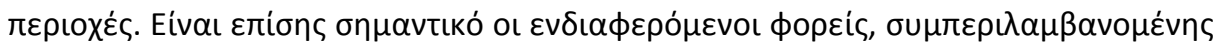

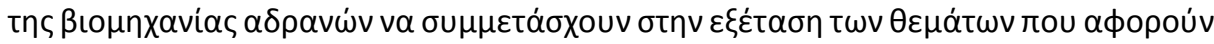

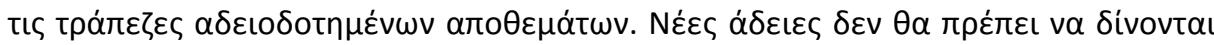

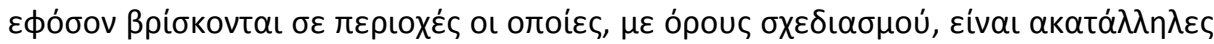

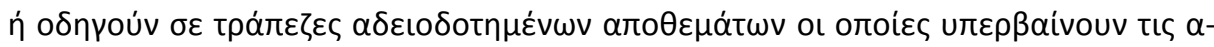

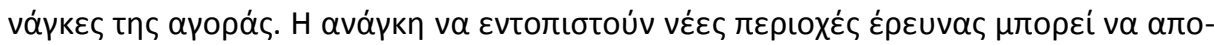

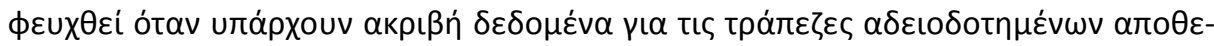

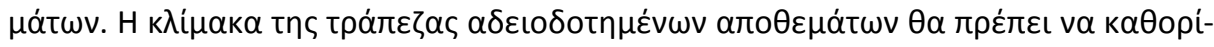

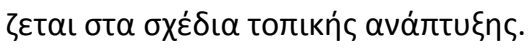

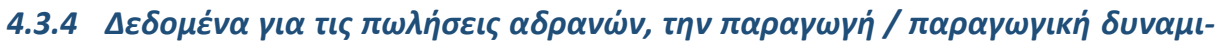

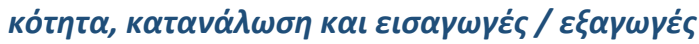

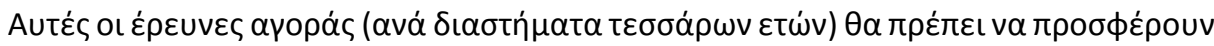

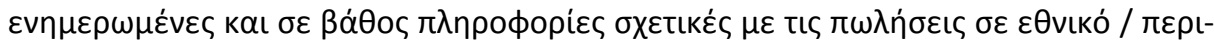




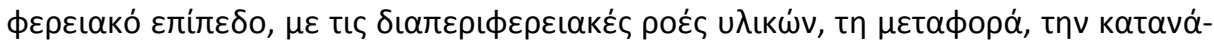

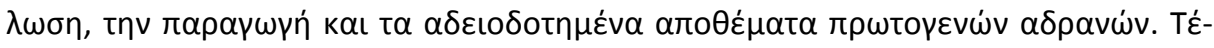

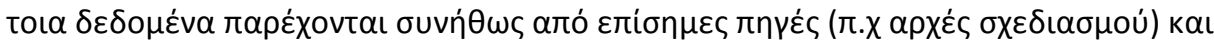

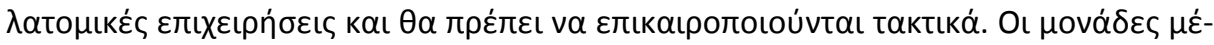

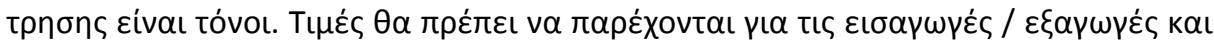

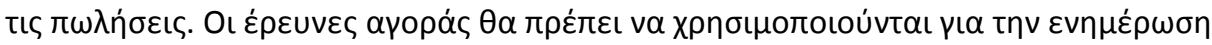

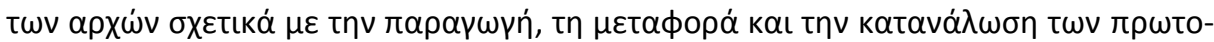

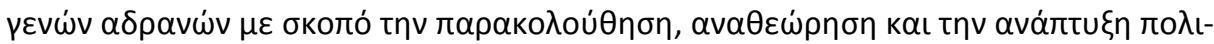

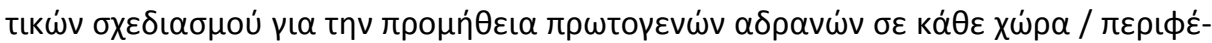
$\rho \varepsilon เ \alpha$.

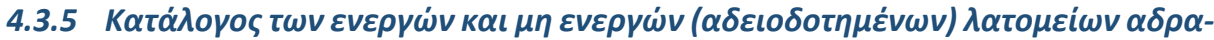 vẃv}

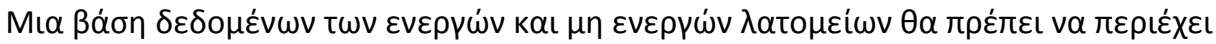

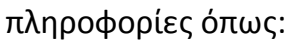

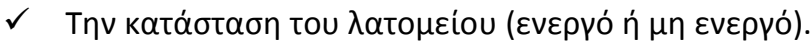

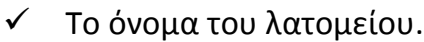

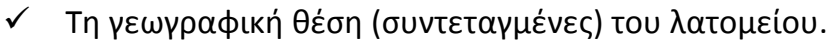

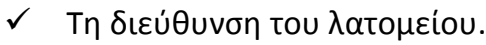

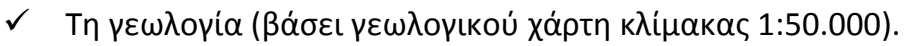

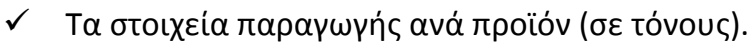

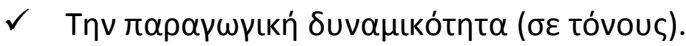

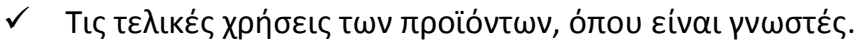

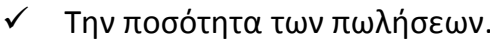

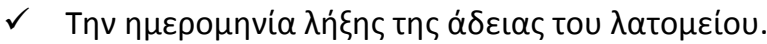

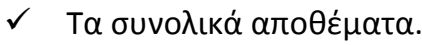

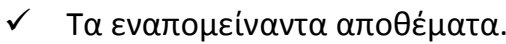

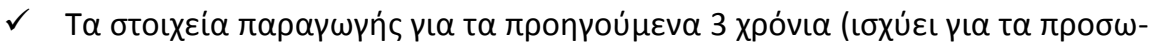

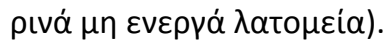

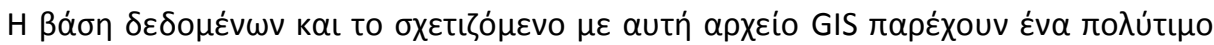

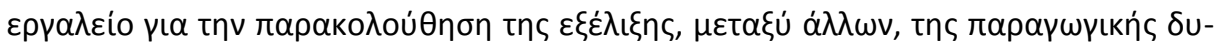

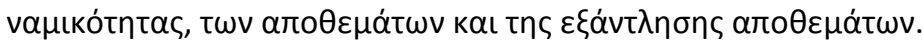

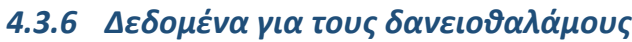

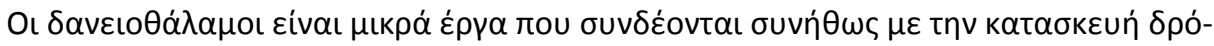

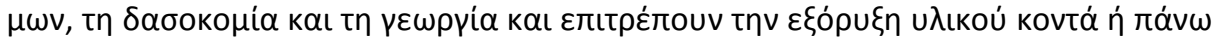

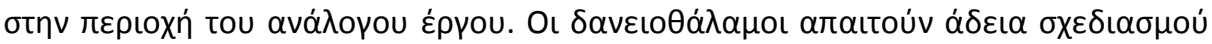




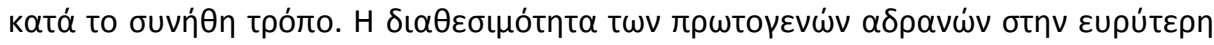

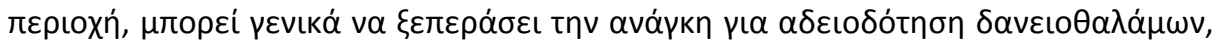

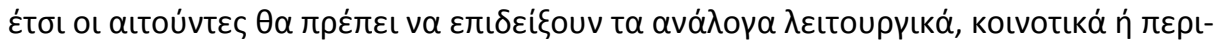

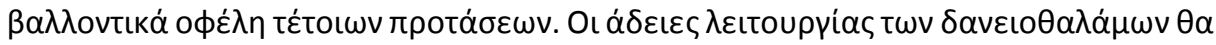

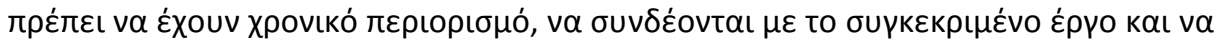

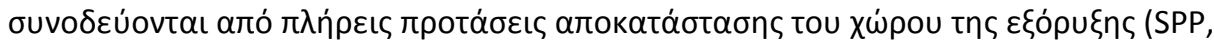
2006).

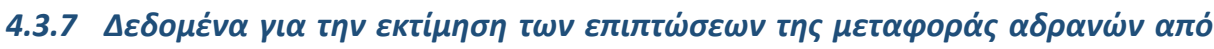

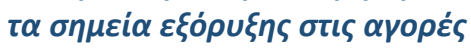

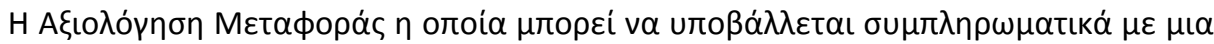

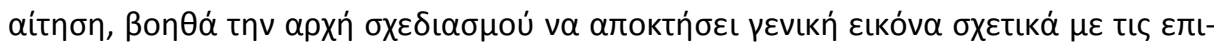

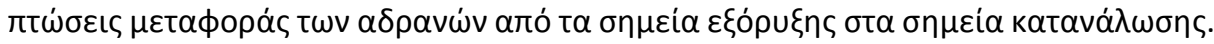

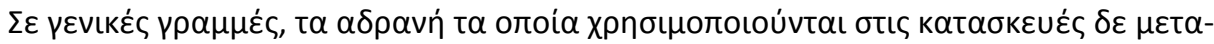

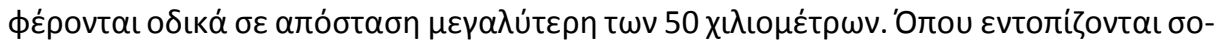

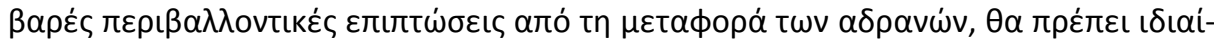

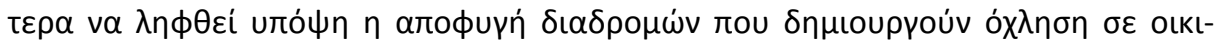

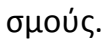

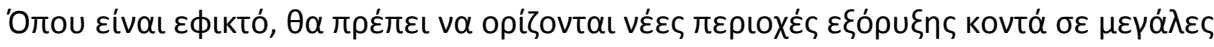

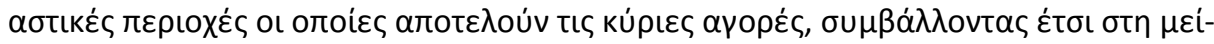

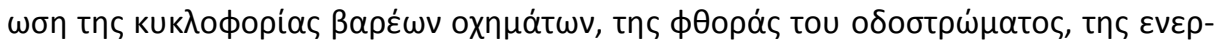

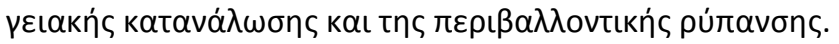

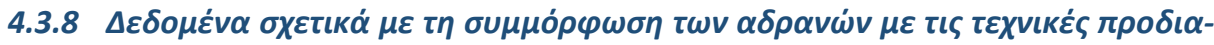

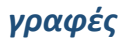

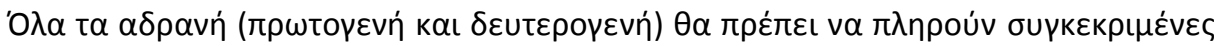

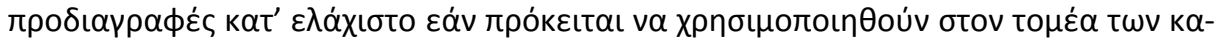

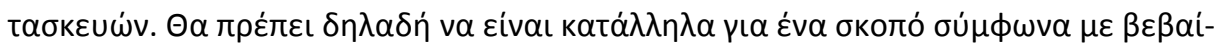

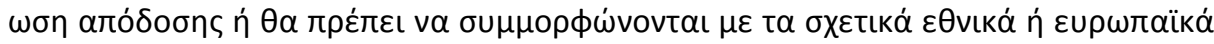

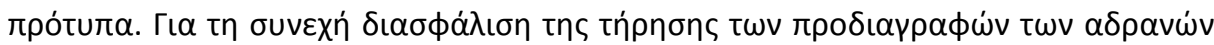

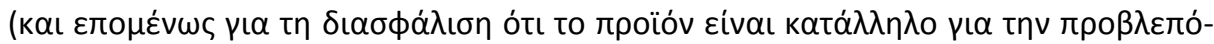

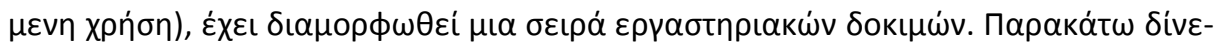

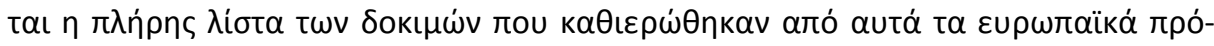

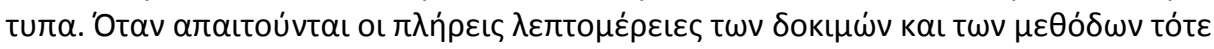

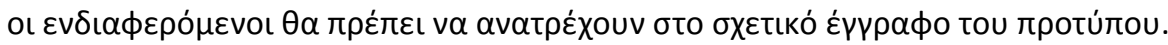

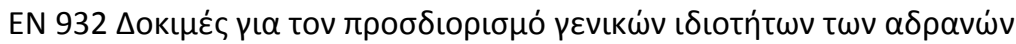

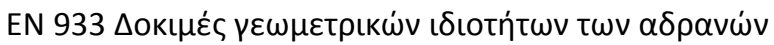




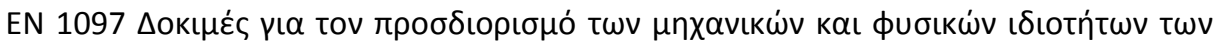
$\alpha \delta \rho a v \omega \dot{v}$

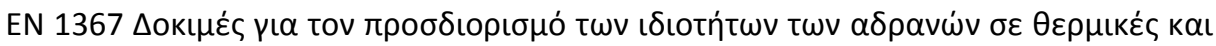

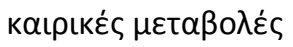

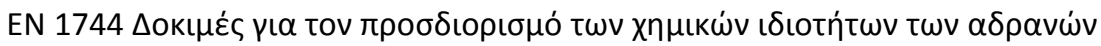

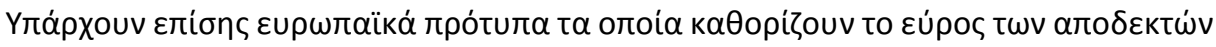

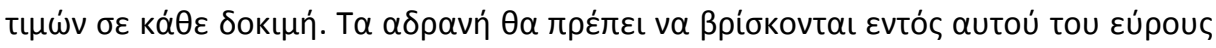

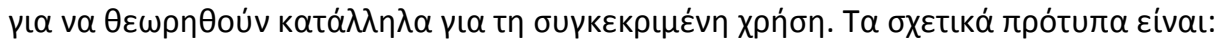

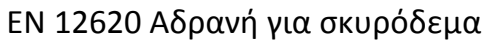

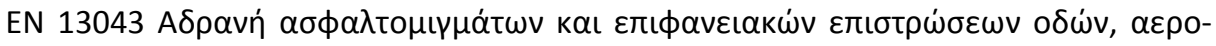

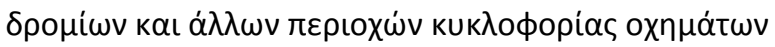

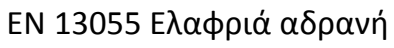

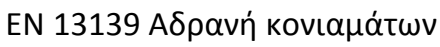

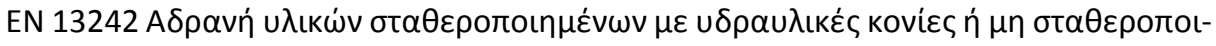

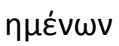

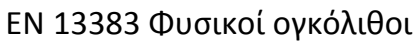

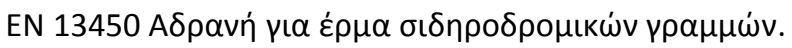

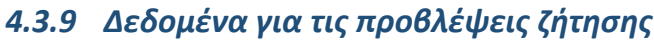

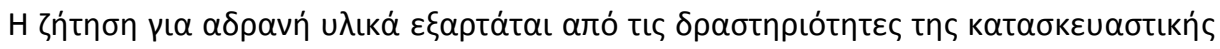

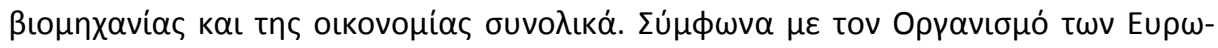

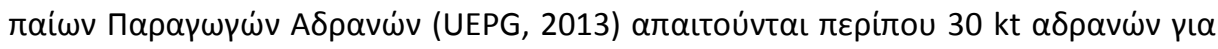

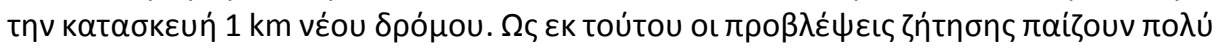

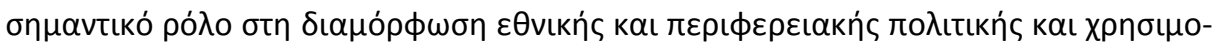

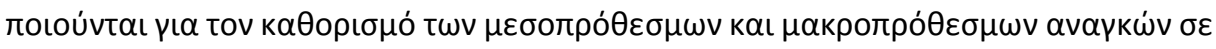

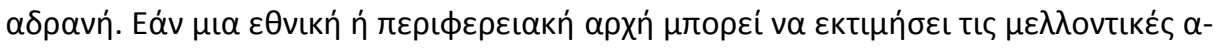

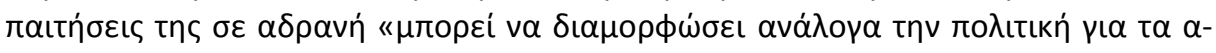

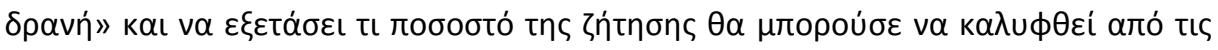

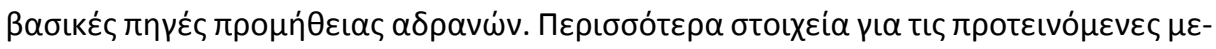

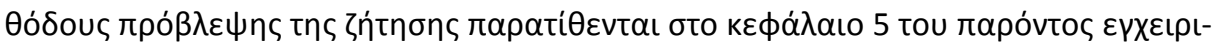
Síou.

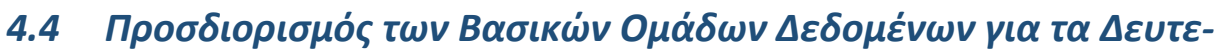

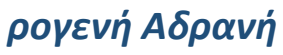

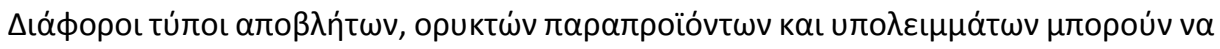

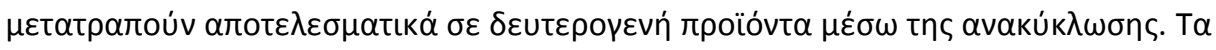

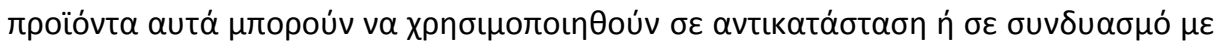

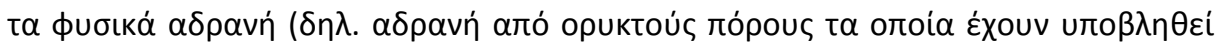




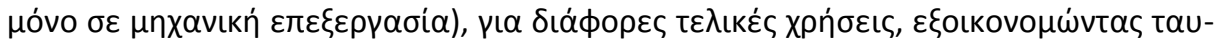

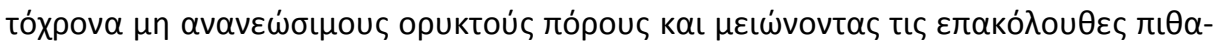

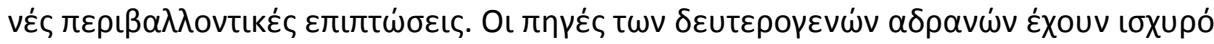

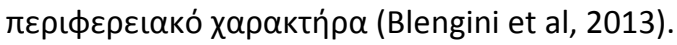

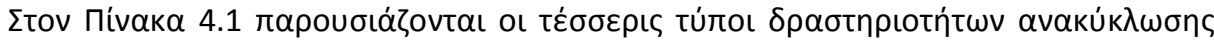

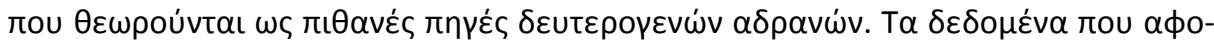

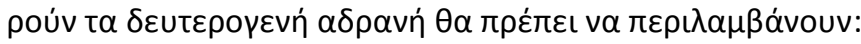

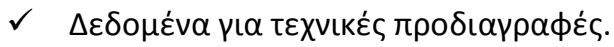

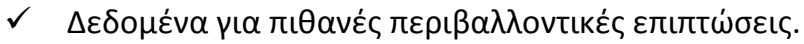

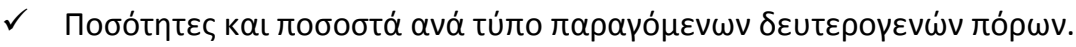

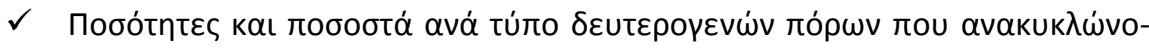
vtat.

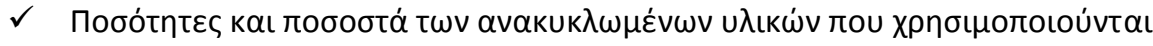

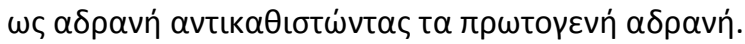

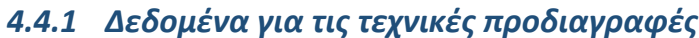

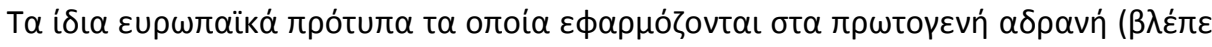

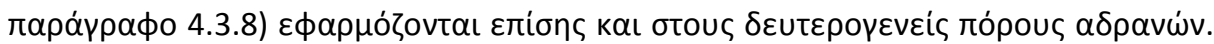

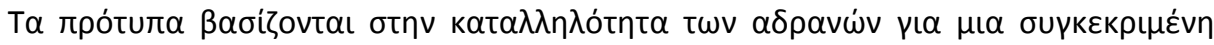

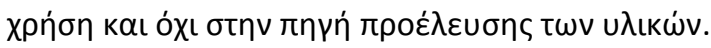

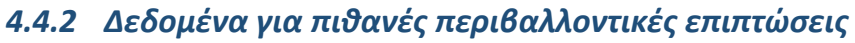

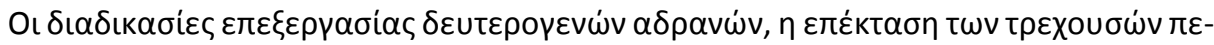

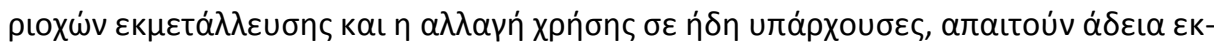

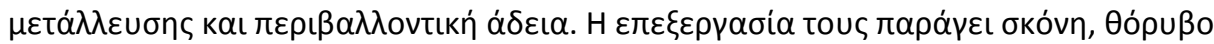

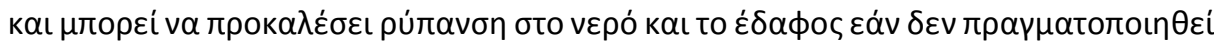

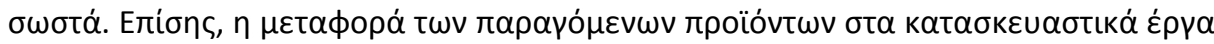

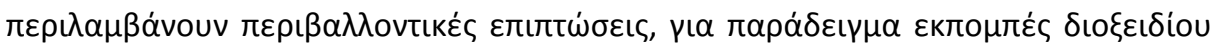

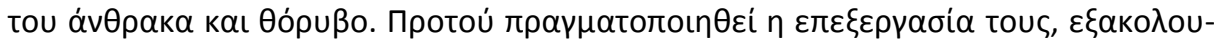

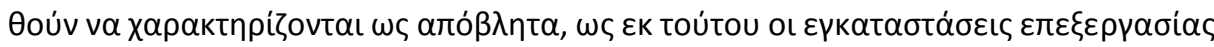

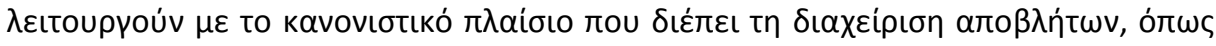

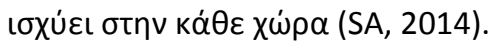

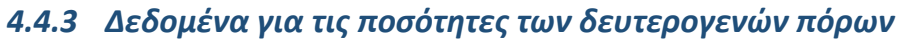

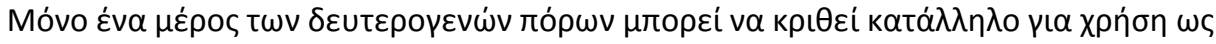
$\alpha \delta \rho \alpha v \varepsilon \dot{s}$ U

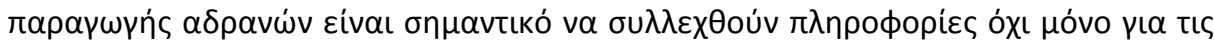

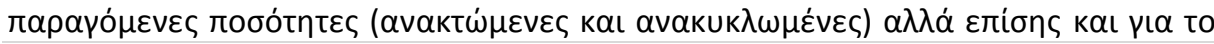




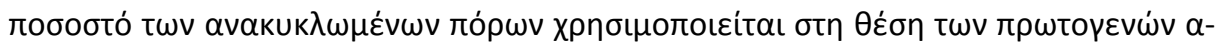

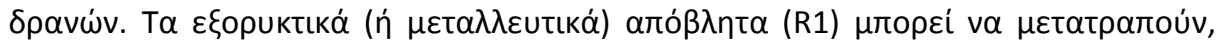

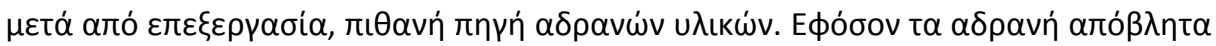

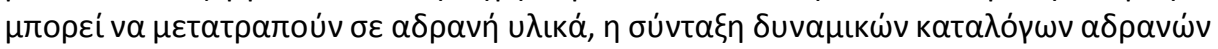

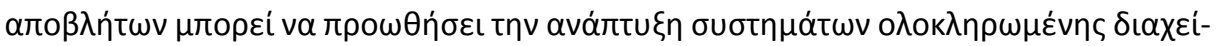

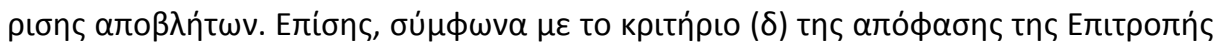

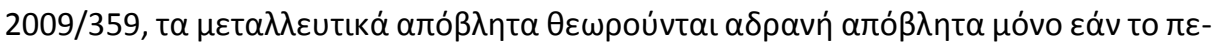

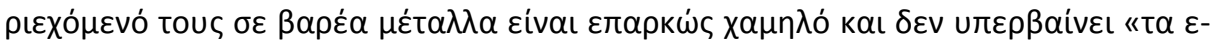

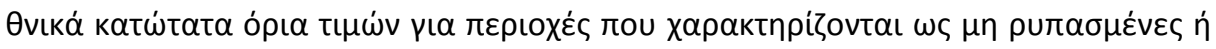

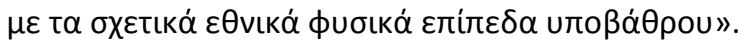

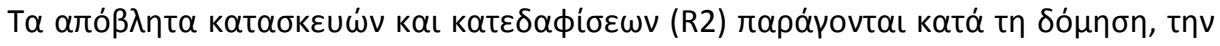

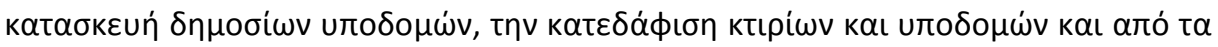

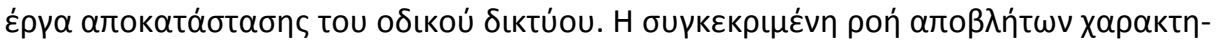

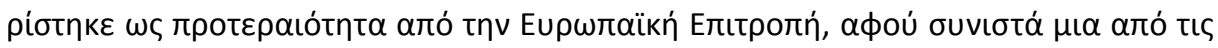

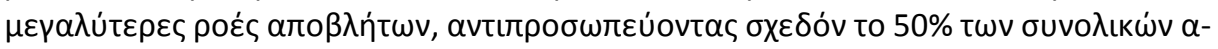

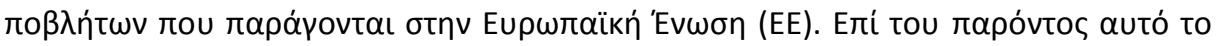

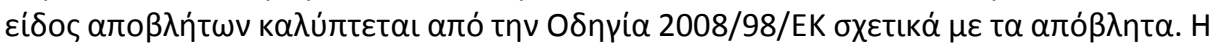

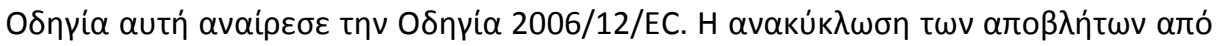

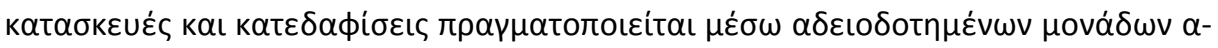

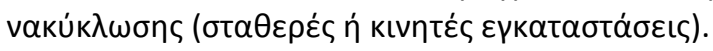

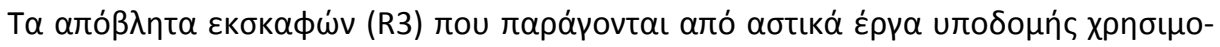

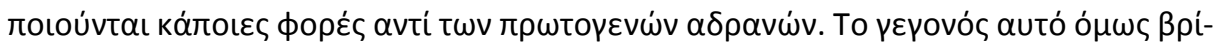

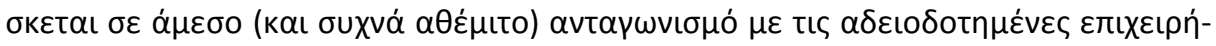

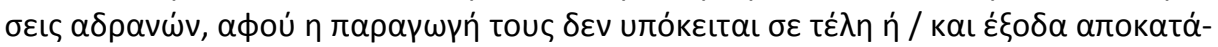

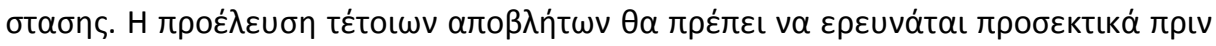

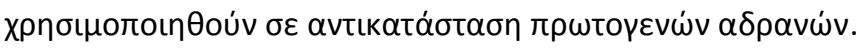

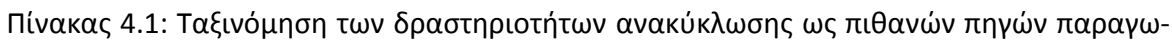

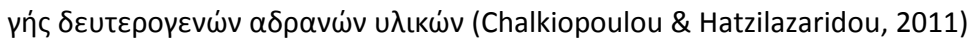

\begin{tabular}{|c|c|}
\hline R1: & 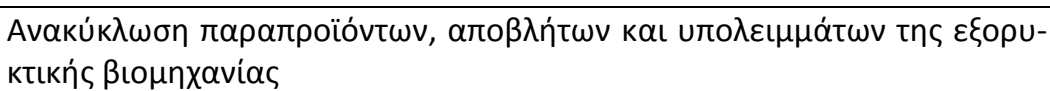 \\
\hline R2: & 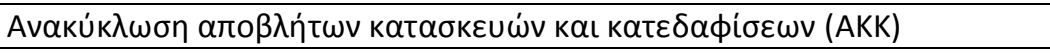 \\
\hline R3: & 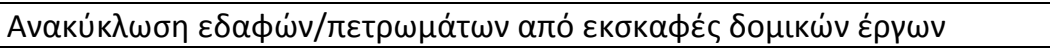 \\
\hline R4: & 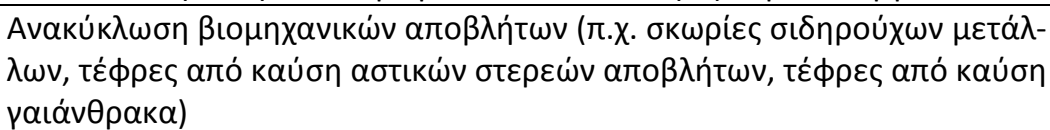 \\
\hline
\end{tabular}




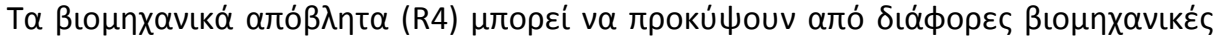

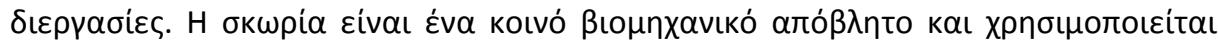

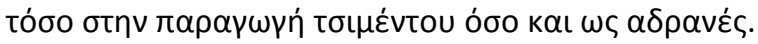

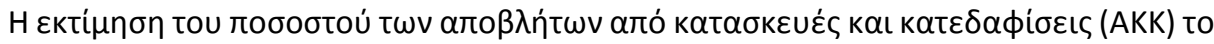

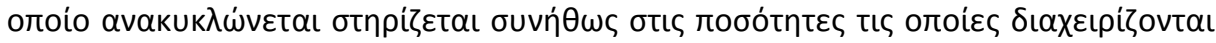

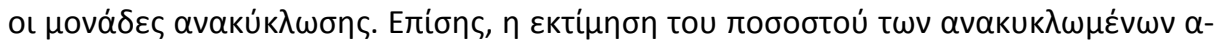

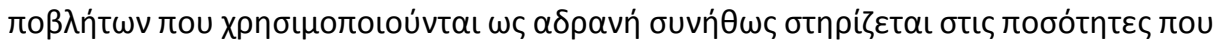
$\pi \omega \lambda \eta \dot{\theta \eta \kappa к а v . ~}$

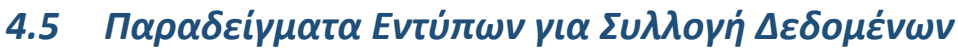

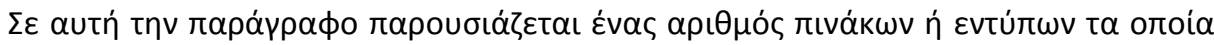

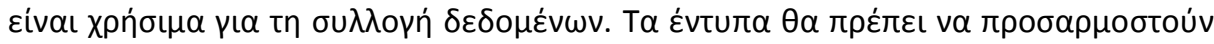

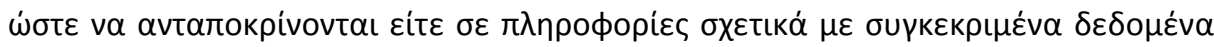

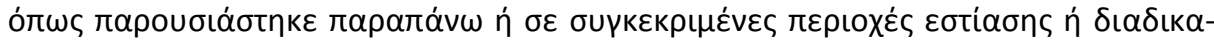

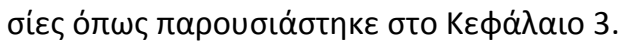

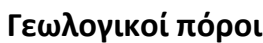

\begin{tabular}{|c|c|c|c|c|c|c|}
\hline 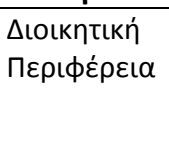 & 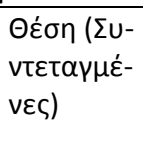 & 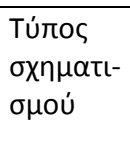 & 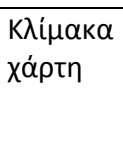 & $\begin{array}{l}\text { АлоӨर́- } \\
\mu \alpha \tau \alpha\end{array}$ & 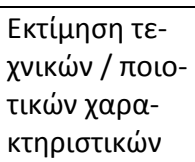 & 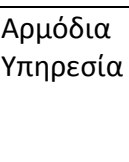 \\
\hline & & & & & & \\
\hline
\end{tabular}

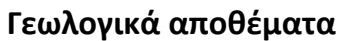

\begin{tabular}{|c|c|c|c|c|c|}
\hline 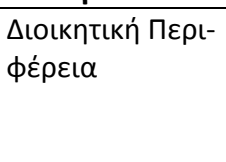 & 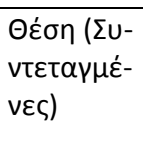 & 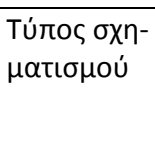 & 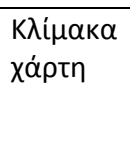 & $\begin{array}{l}\text { АлоӨв́- } \\
\mu \alpha \tau \alpha\end{array}$ & 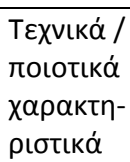 \\
\hline 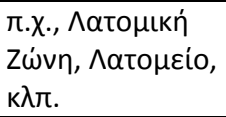 & & & & & \\
\hline
\end{tabular}




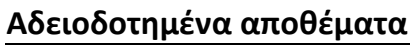

\begin{tabular}{|c|c|c|c|c|c|c|}
\hline 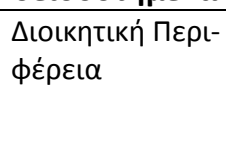 & 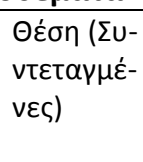 & 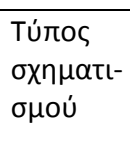 & $\begin{array}{l}\text { K入i- } \\
\mu \alpha \kappa \alpha \\
\chi \alpha \dot{\alpha} \rho \tau \eta\end{array}$ & $\begin{array}{l}\text { АлоӨध́- } \\
\mu \alpha \tau \alpha\end{array}$ & 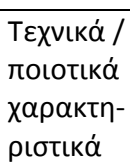 & 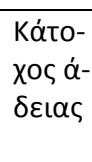 \\
\hline 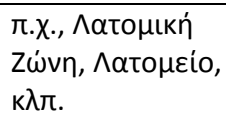 & & & & & & \\
\hline
\end{tabular}

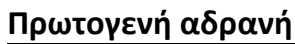

\begin{tabular}{|c|c|c|c|c|c|}
\hline 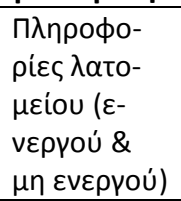 & 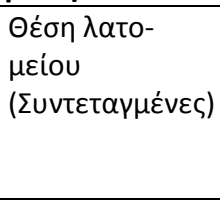 & 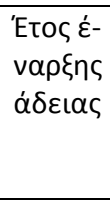 & $\begin{array}{l}\text { 'Etos } \lambda n^{-}- \\
\xi n \varsigma \alpha \alpha_{-} \\
\delta \varepsilon l \alpha \varsigma\end{array}$ & $\begin{array}{l}\text { Túrol } \sigma \times \eta- \\
\mu \alpha \tau \imath \mu \omega \dot{v}\end{array}$ & 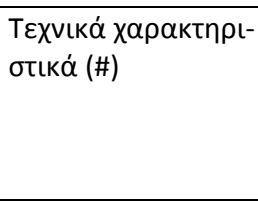 \\
\hline 1. & & & & 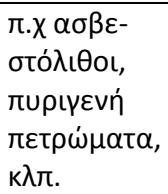 & 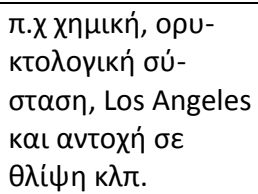 \\
\hline
\end{tabular}

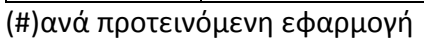

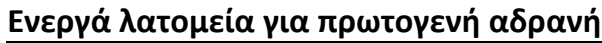

\begin{tabular}{|c|c|c|c|c|c|}
\hline 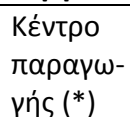 & 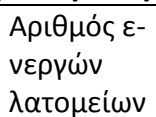 & $\begin{array}{l}\text { 'Eтоৎ } \alpha v \alpha- \\
\text { фора́s }\end{array}$ & 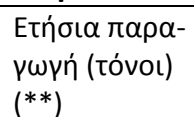 & 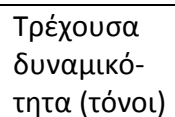 & 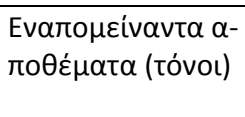 \\
\hline \multirow[t]{3}{*}{1} & 1.1 & & & & \\
\hline & 1.2 & & & & \\
\hline & $1 . n$ & & & & \\
\hline \multirow[t]{2}{*}{2} & 2.1 & & & & \\
\hline & $2 . n$ & & & & \\
\hline
\end{tabular}

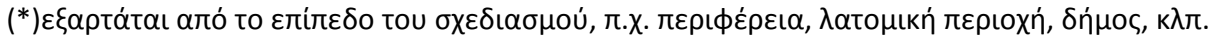

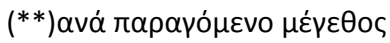

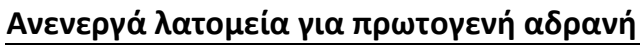

\begin{tabular}{|c|c|c|c|c|}
\hline 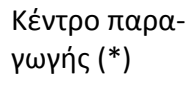 & 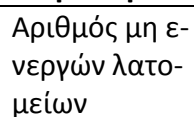 & 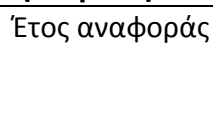 & 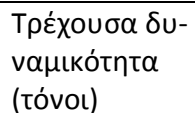 & 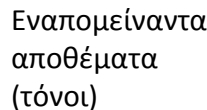 \\
\hline
\end{tabular}

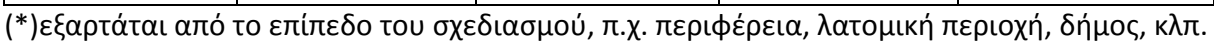




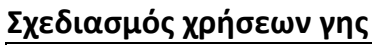

\begin{tabular}{|c|c|c|}
\hline 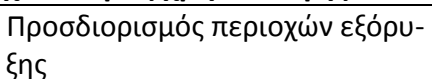 & 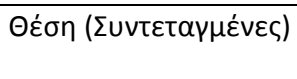 & 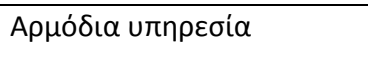 \\
\hline 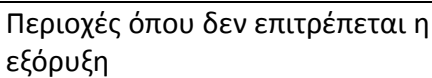 & & 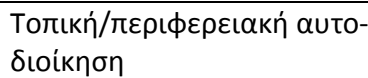 \\
\hline 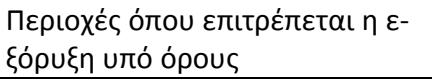 & & \\
\hline 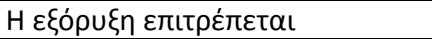 & & \\
\hline 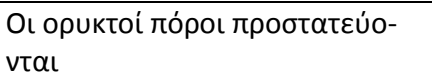 & & \\
\hline
\end{tabular}

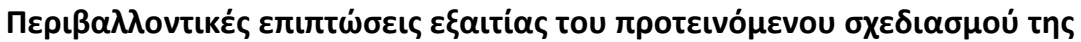

\section{$\pi \alpha \rho \alpha \gamma \omega \gamma \eta \dot{\varsigma} \alpha \delta \rho \alpha v \omega \dot{v}$}

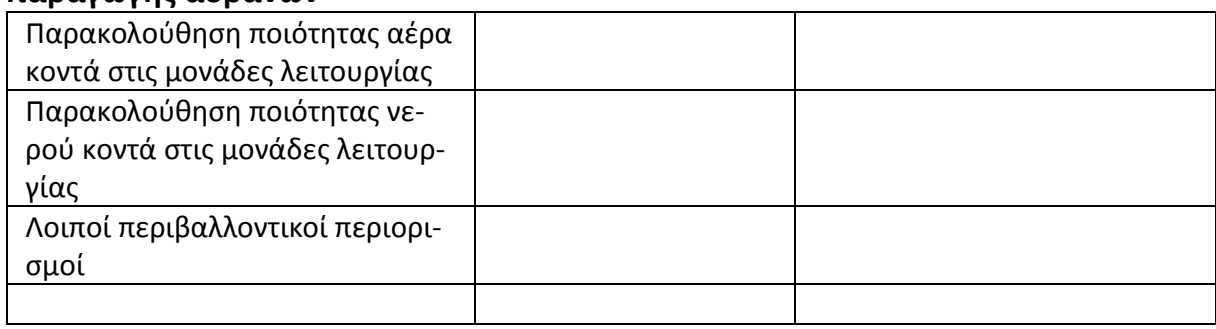

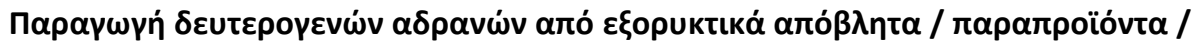
Uாо

\begin{tabular}{|c|c|c|c|c|c|}
\hline 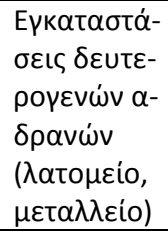 & 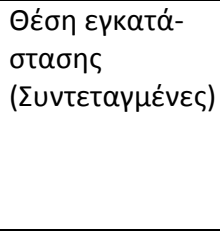 & 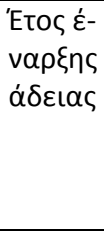 & $\begin{array}{l}\text { 'Etos } \lambda \dot{-}- \\
\xi n \varsigma \dot{\alpha}- \\
\delta \varepsilon l \alpha \varsigma\end{array}$ & 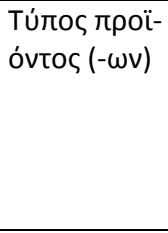 & 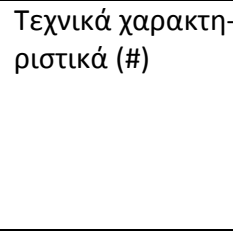 \\
\hline 1. & & & & & 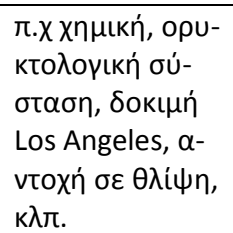 \\
\hline
\end{tabular}

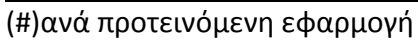




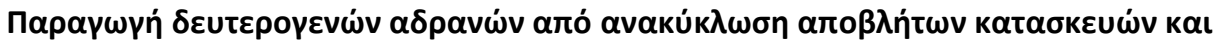

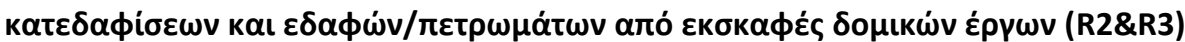

\begin{tabular}{|c|c|c|c|c|c|}
\hline 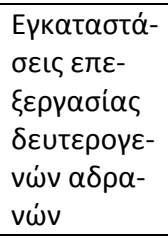 & 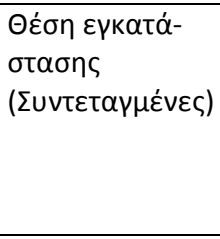 & 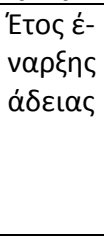 & 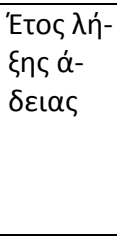 & 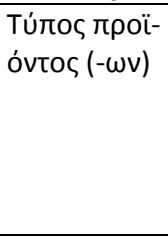 & 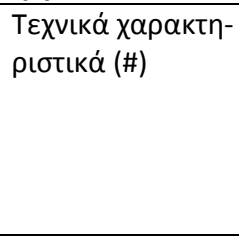 \\
\hline 1. & & & & & 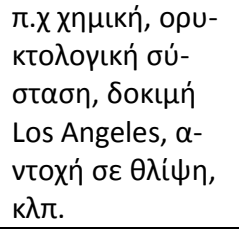 \\
\hline
\end{tabular}

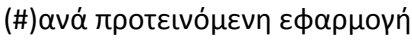

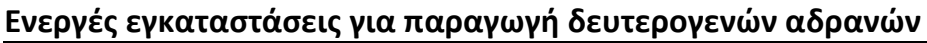

\begin{tabular}{|c|c|c|c|c|}
\hline $\begin{array}{l}\text { Eүк } \alpha \tau^{\prime}{ }^{-}- \\
\sigma \tau \alpha \sigma \eta\left(^{*}\right)\end{array}$ & 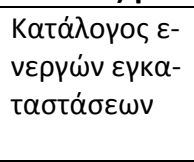 & 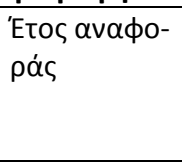 & 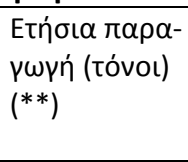 & 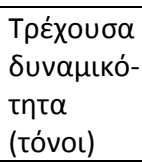 \\
\hline 1 & 1 & & & \\
\hline
\end{tabular}

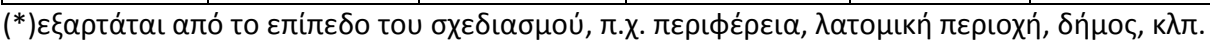




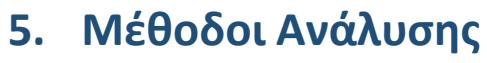

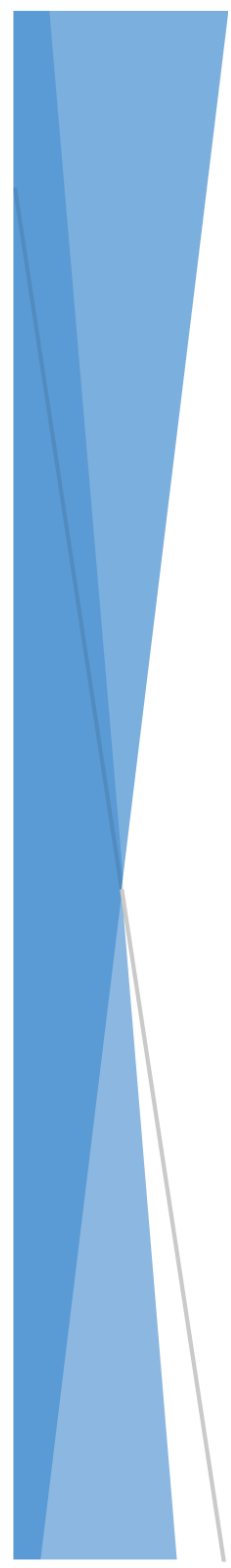

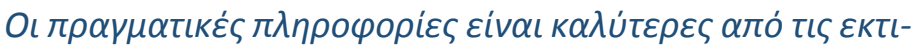

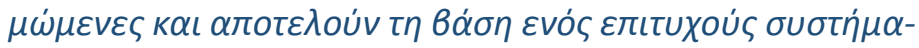

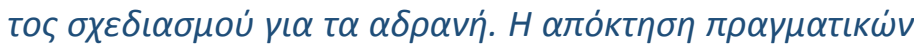

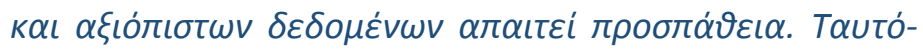

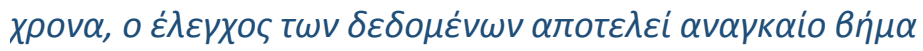

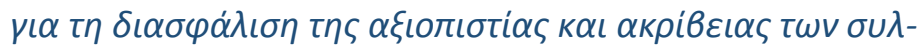

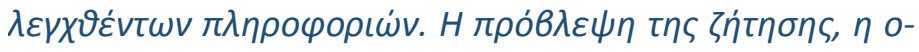

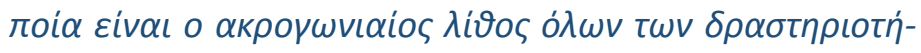

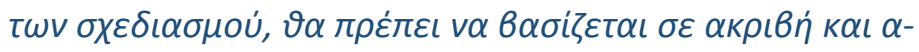

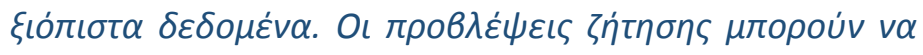

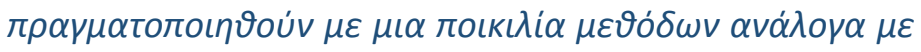

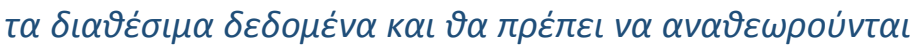

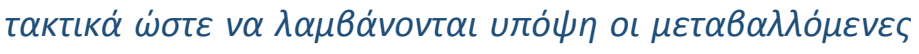

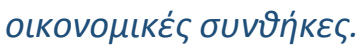




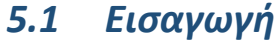

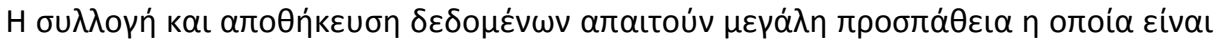

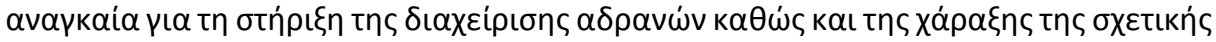

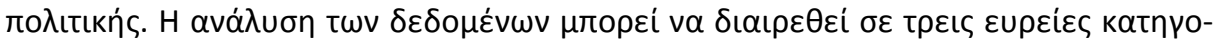

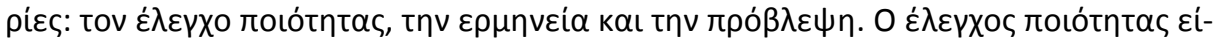

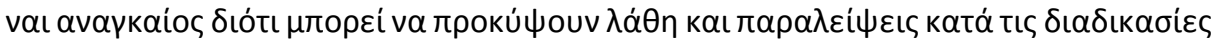

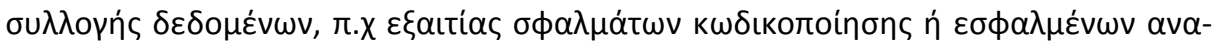

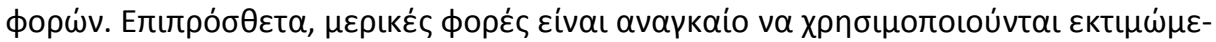

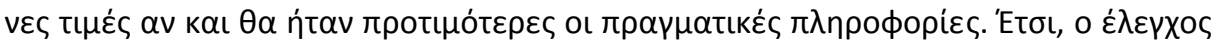

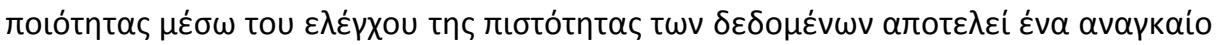

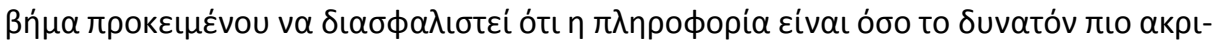

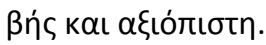

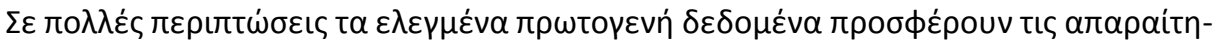

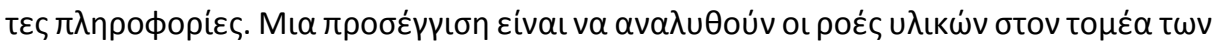

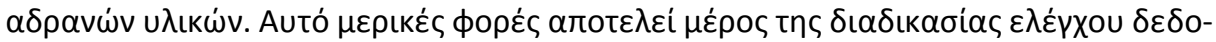

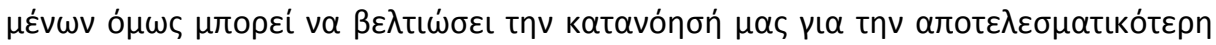

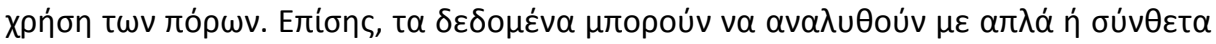

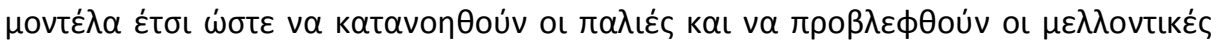

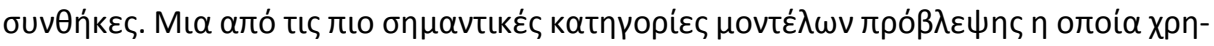

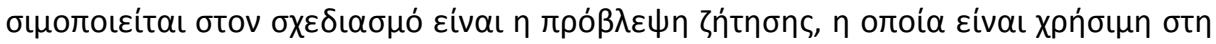

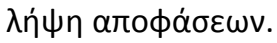

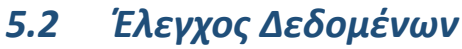

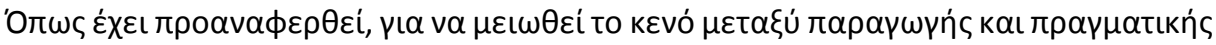

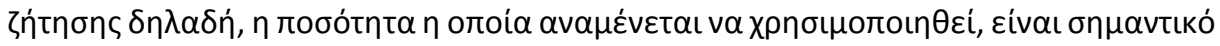

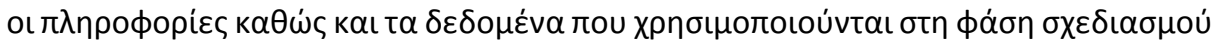

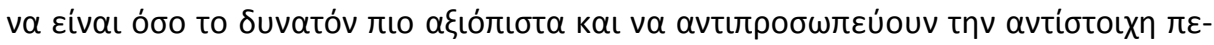

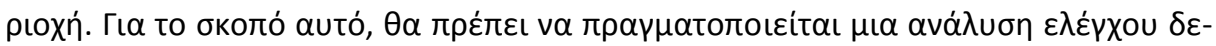

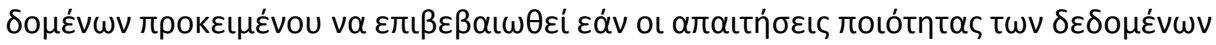

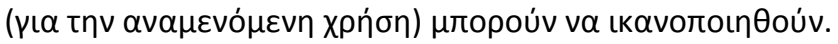

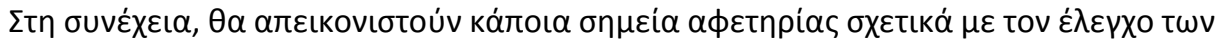

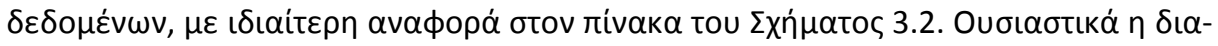

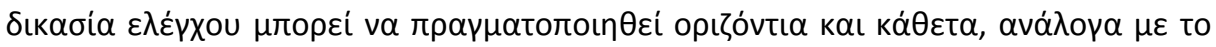

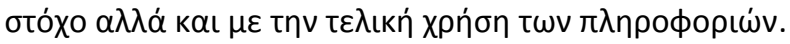




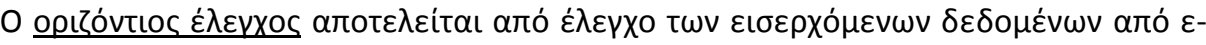

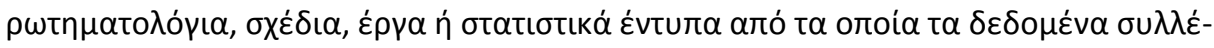

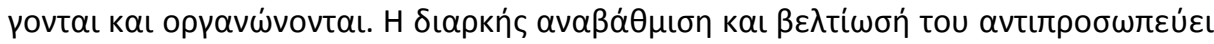

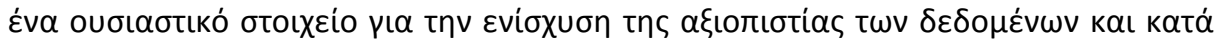

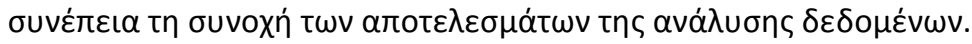

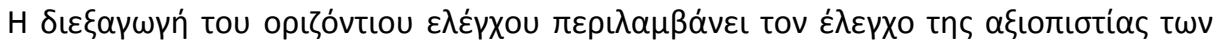

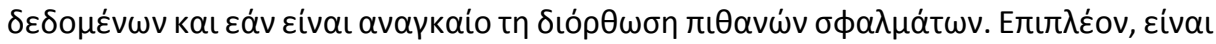

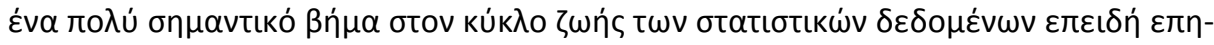

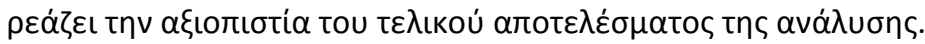

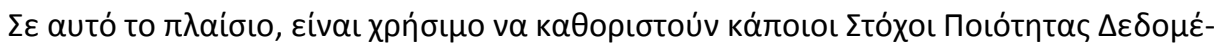

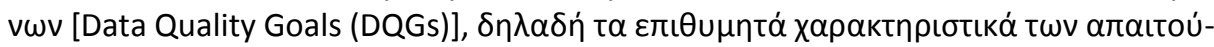

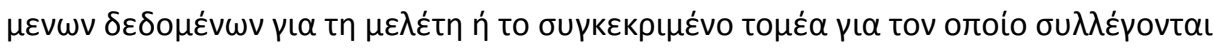

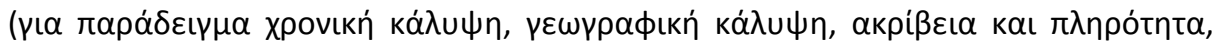

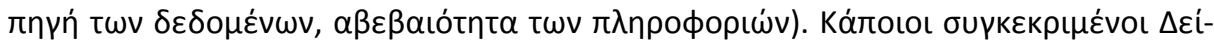

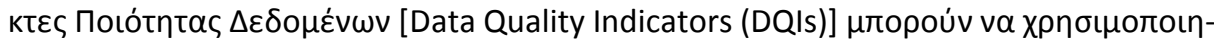

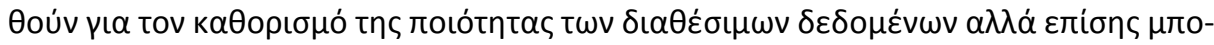

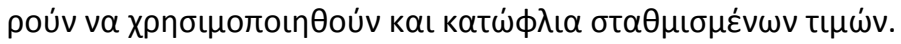

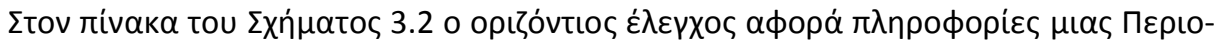

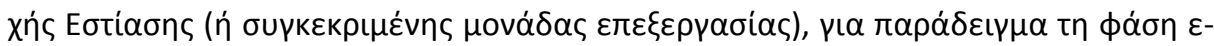

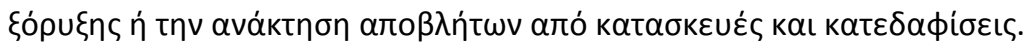

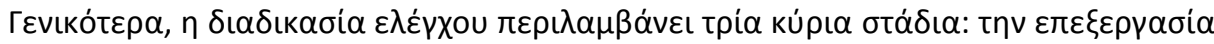

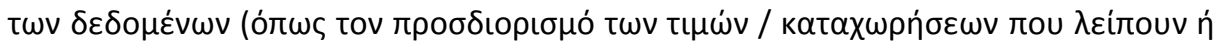

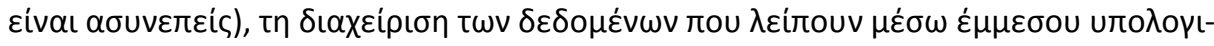

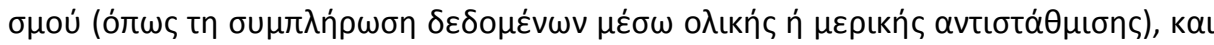

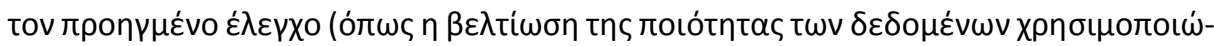

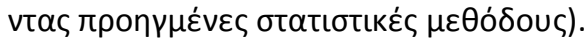

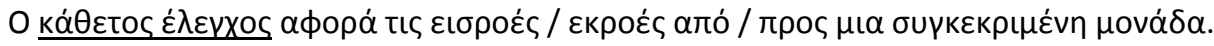

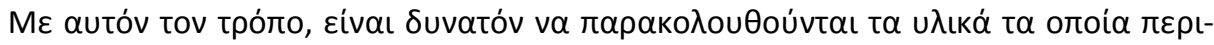

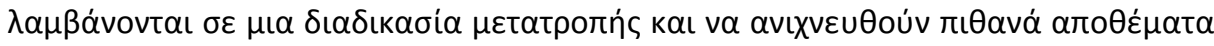

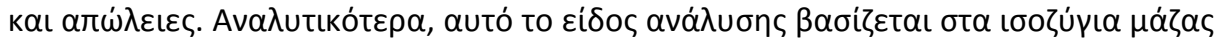

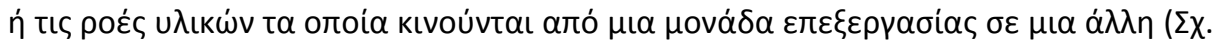

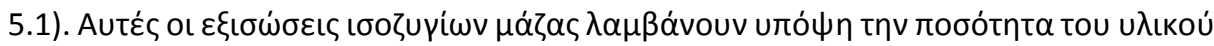

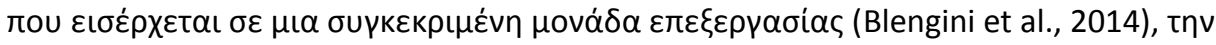

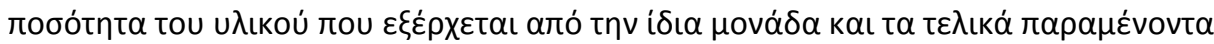

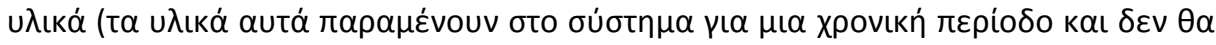

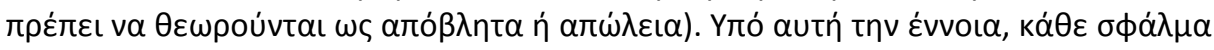




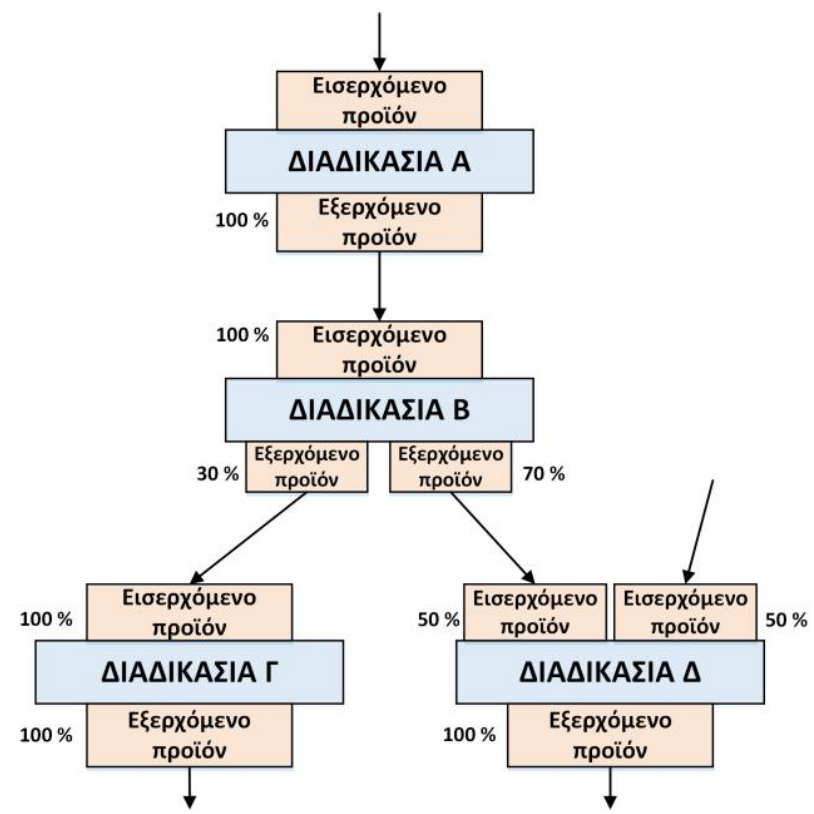

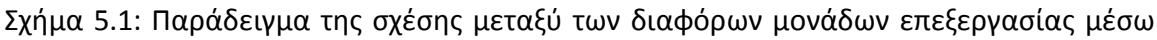

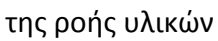

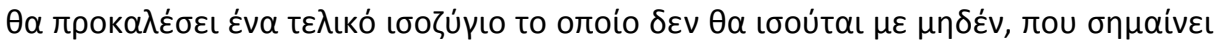

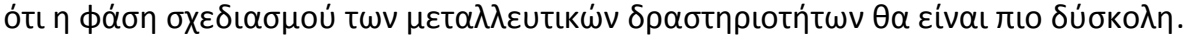

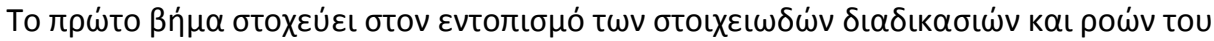

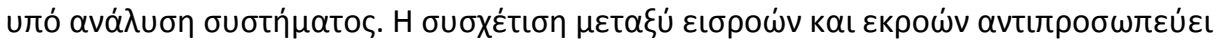

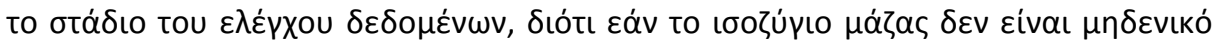

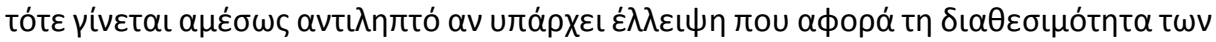

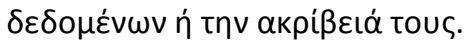

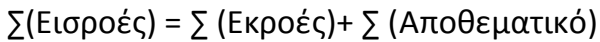

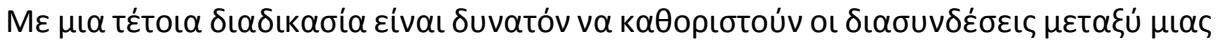

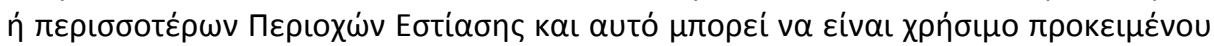

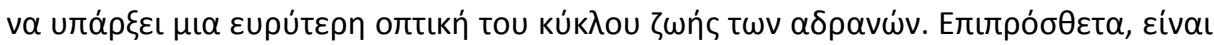

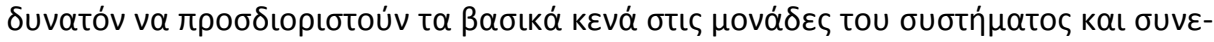

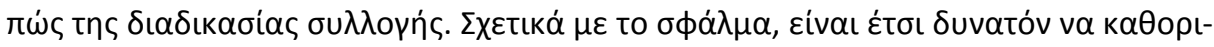

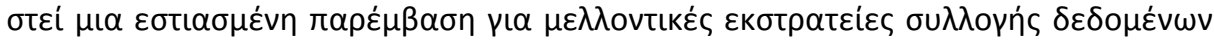

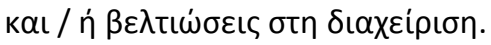




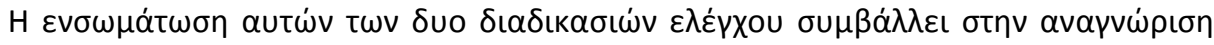

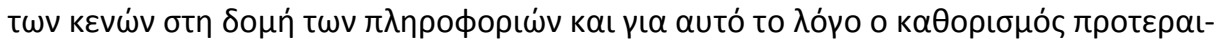

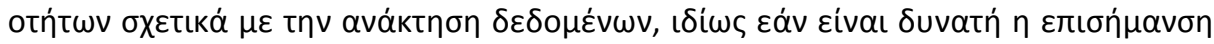

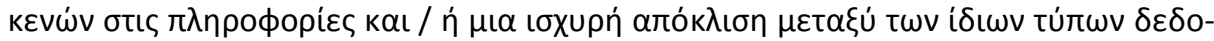

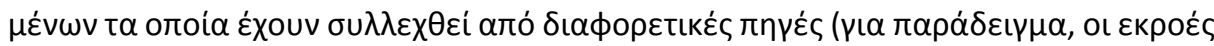

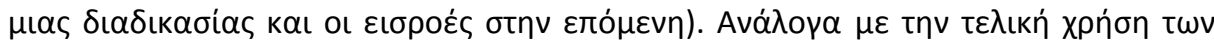

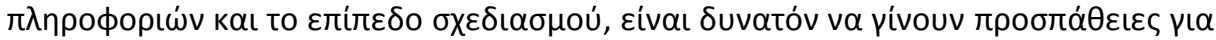

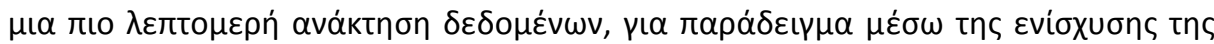

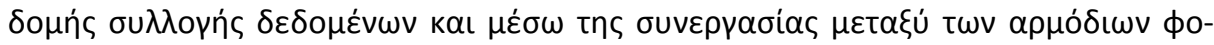

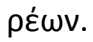

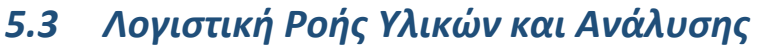

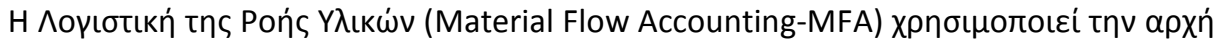

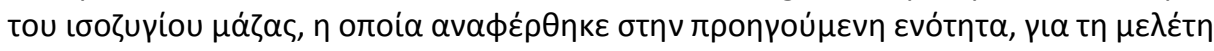

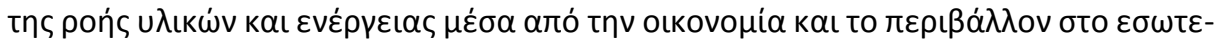

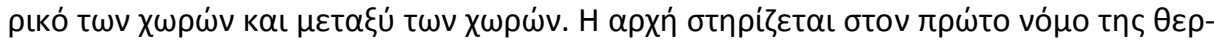

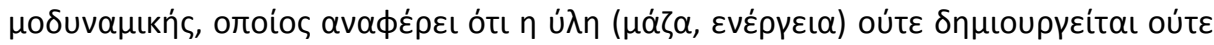

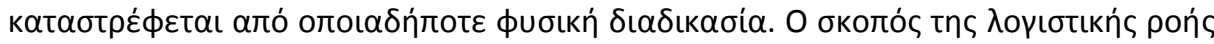

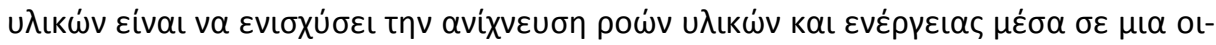

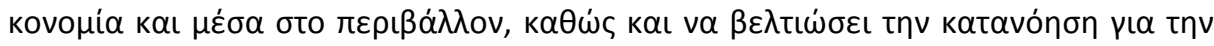

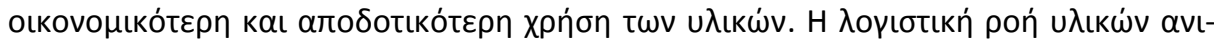

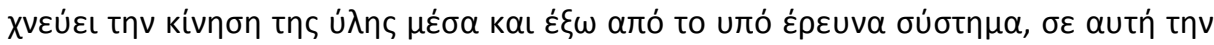

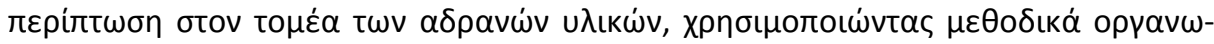

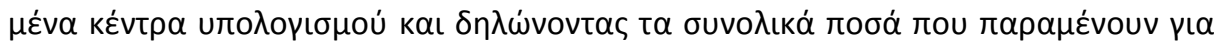

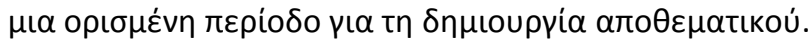

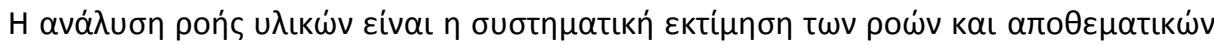

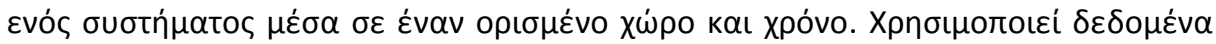

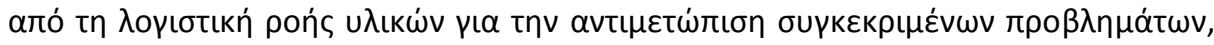

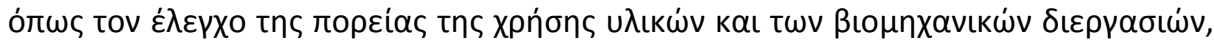

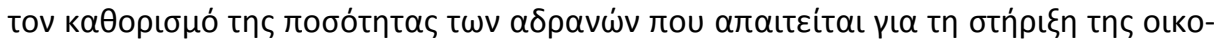

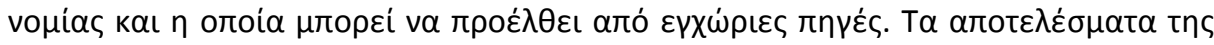

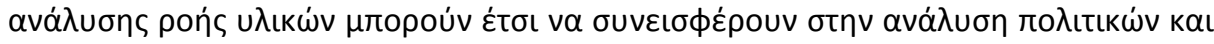

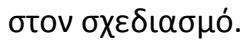

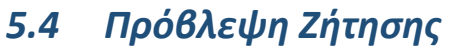

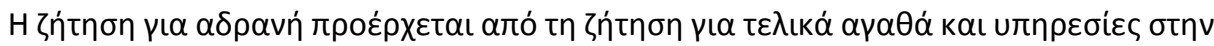

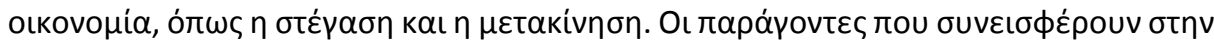




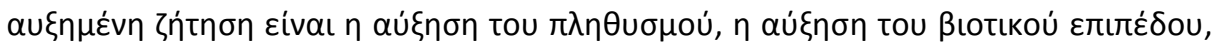

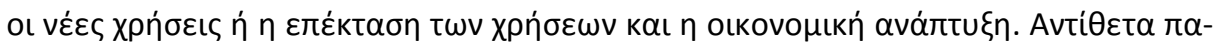

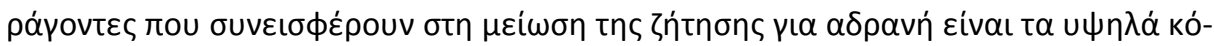

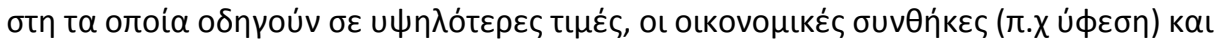

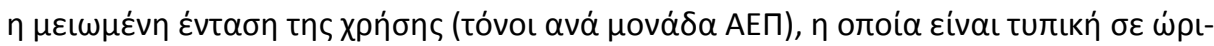

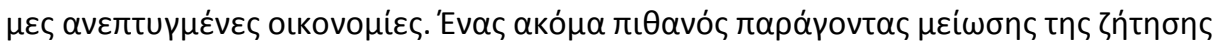

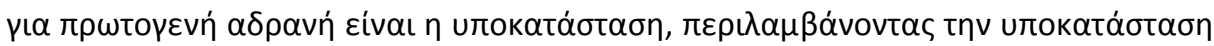

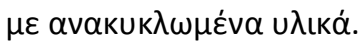

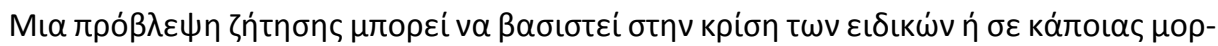

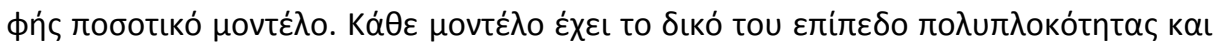
$\lambda \varepsilon \pi \tau$

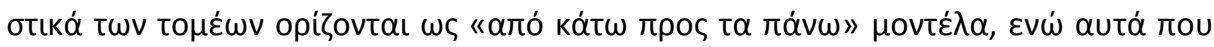

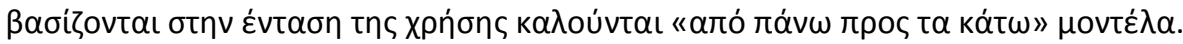

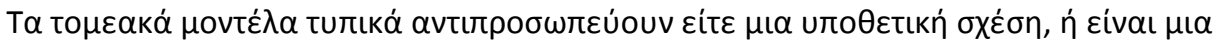

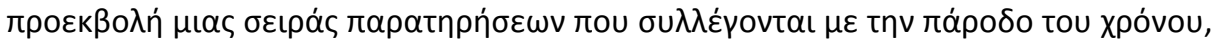

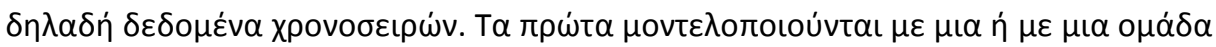

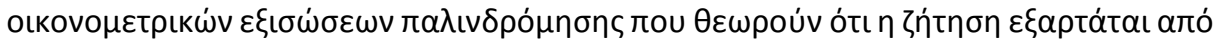

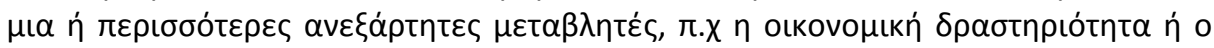

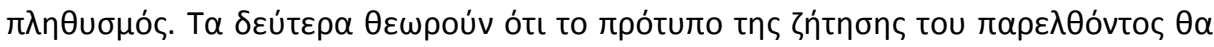

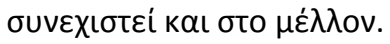

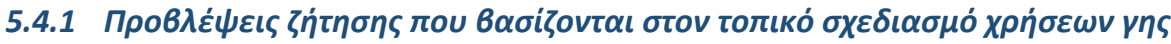

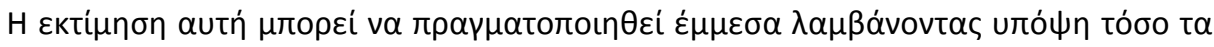

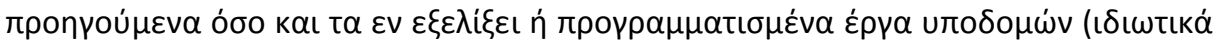

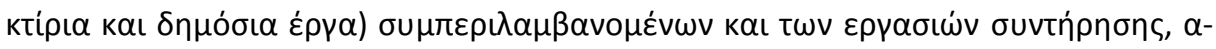

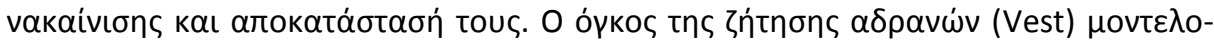

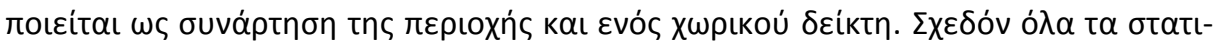

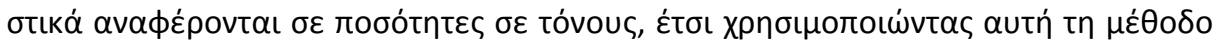

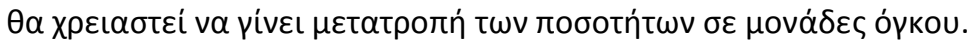

Vest $=($ Sha - Ped $) *$ It

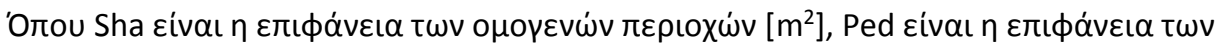

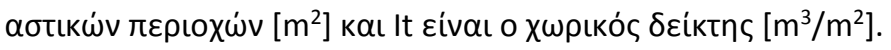

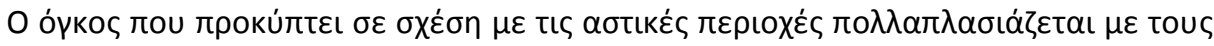

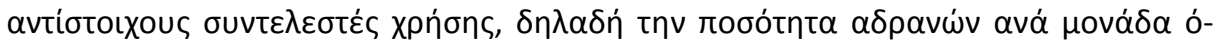

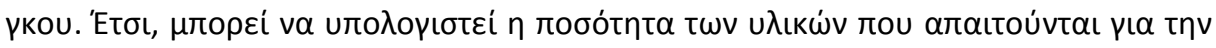




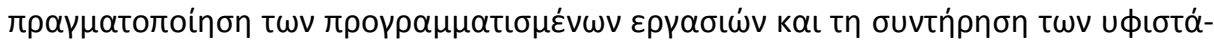

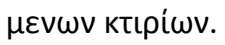

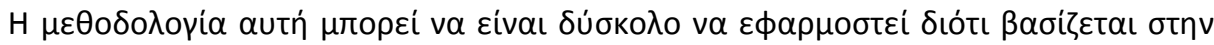

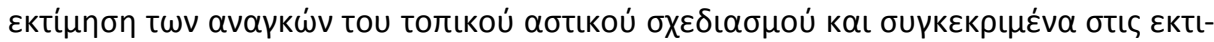

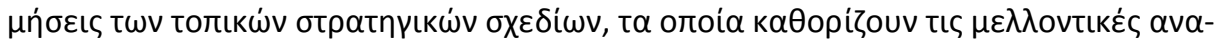

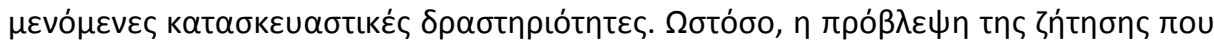

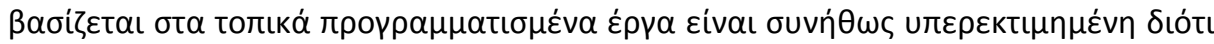

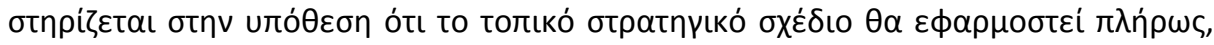

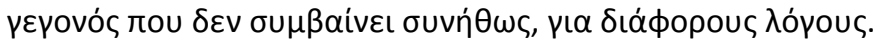

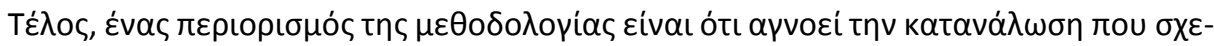

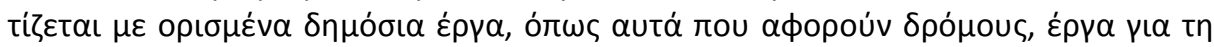

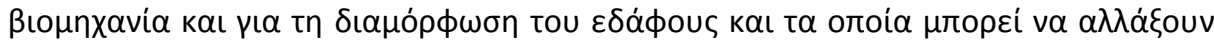

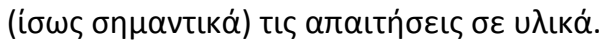

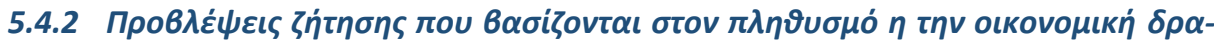

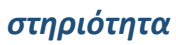

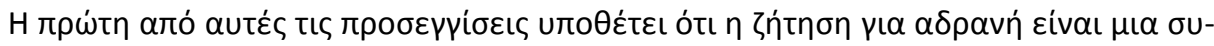

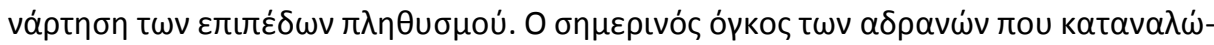

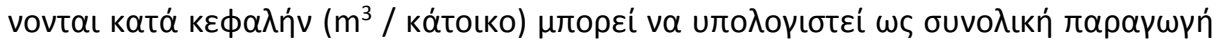

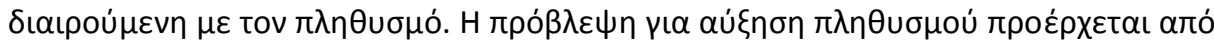

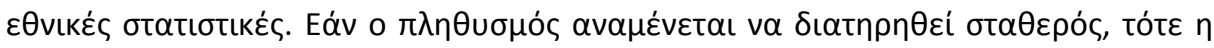

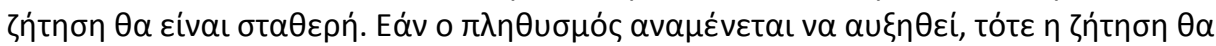

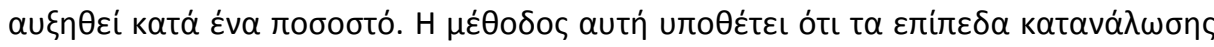

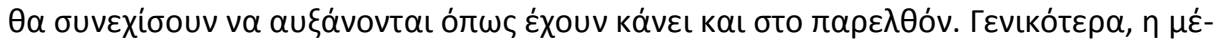

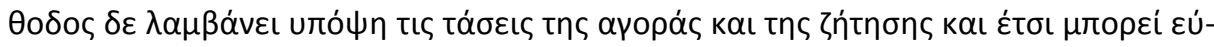

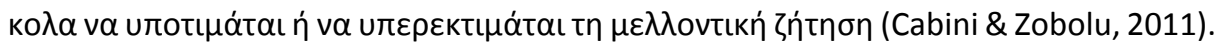

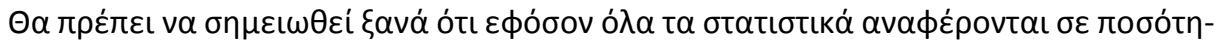

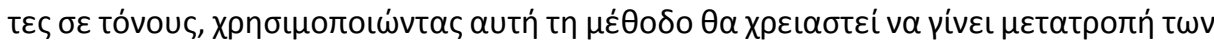

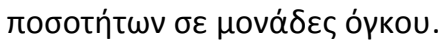

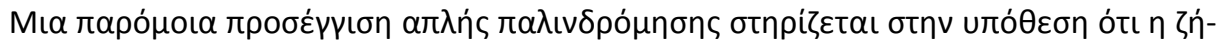

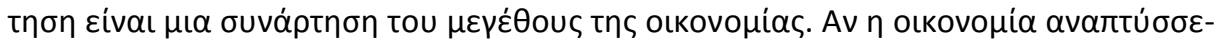

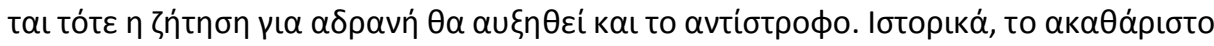

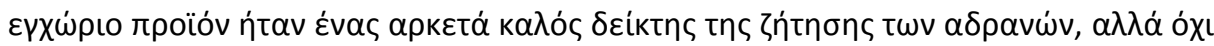

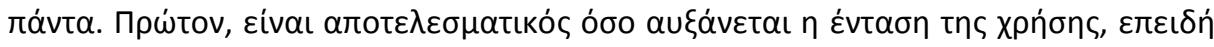

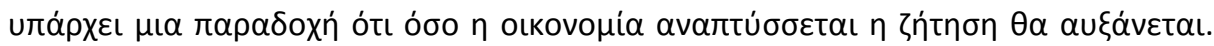

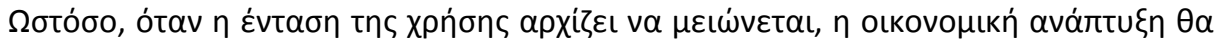

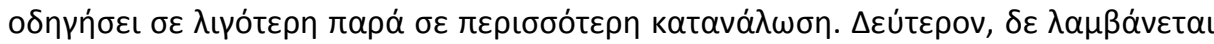




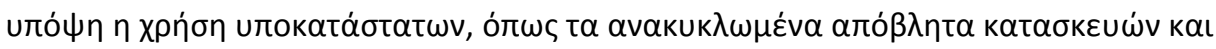

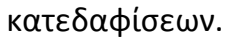

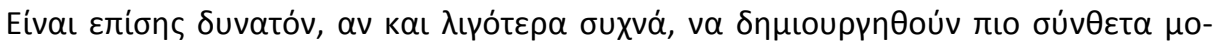

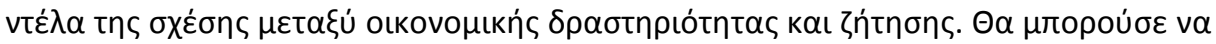

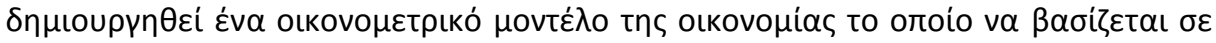

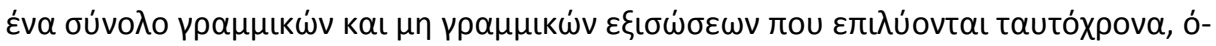

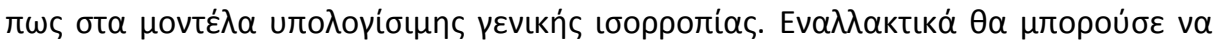

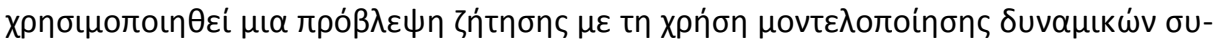

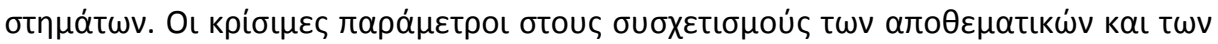

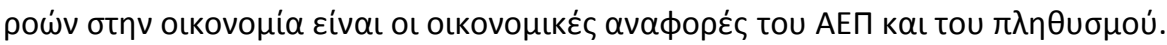

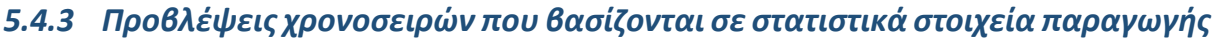

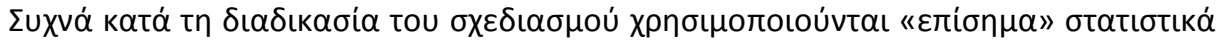

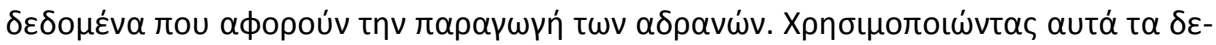

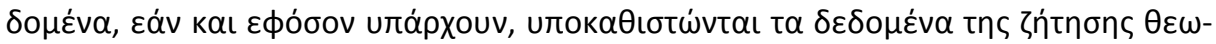

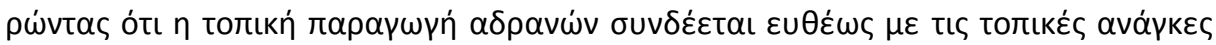

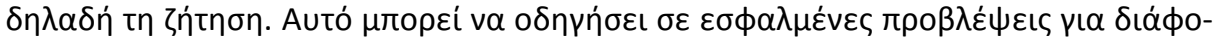
pous $\lambda$ ópous:

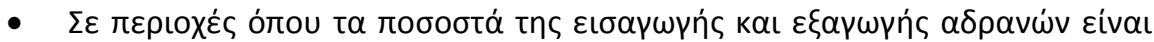

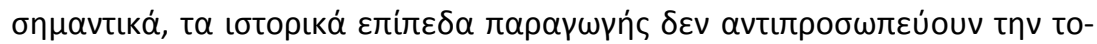

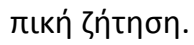

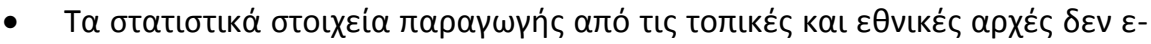

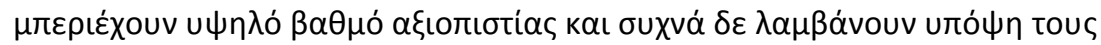

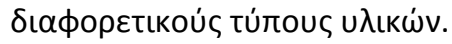

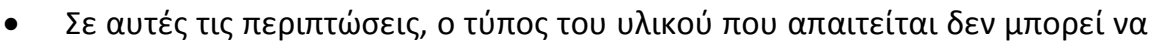

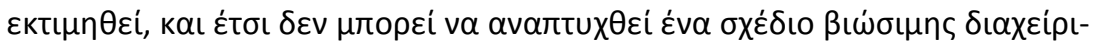

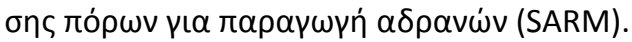

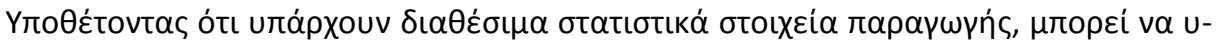

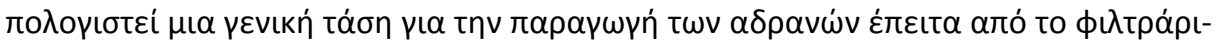

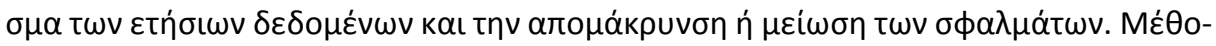

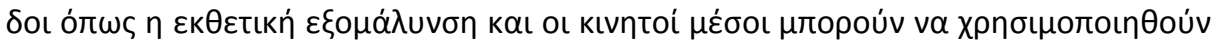

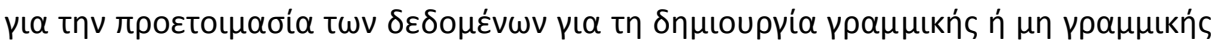

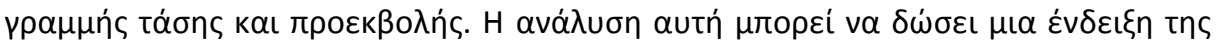

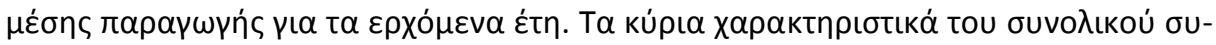

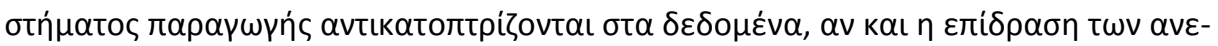

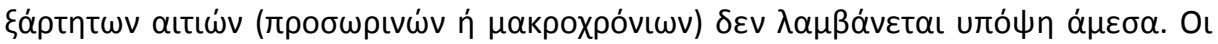




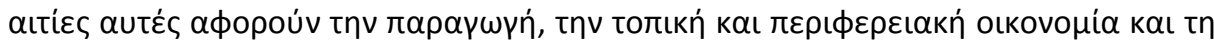
форо入оүía.

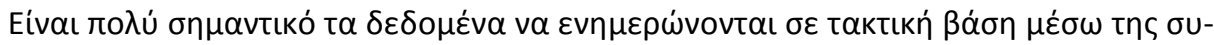

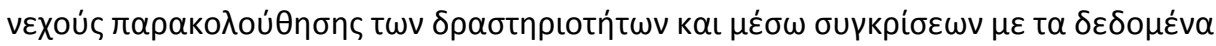

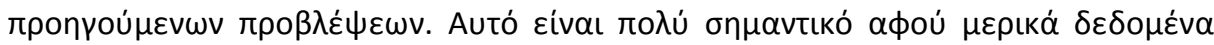

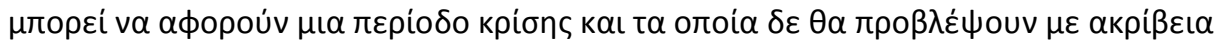

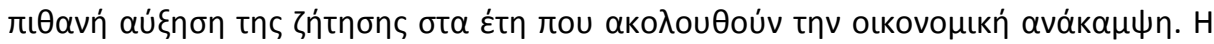

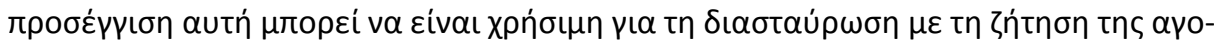

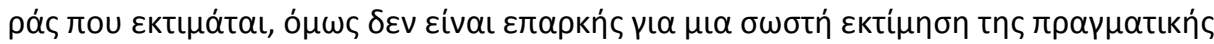

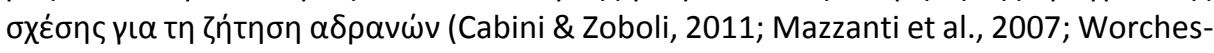
tershire County Council, 2013).

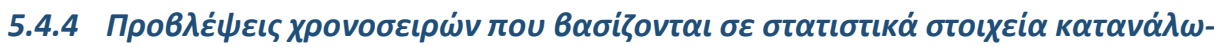 ons}

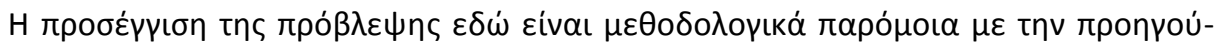

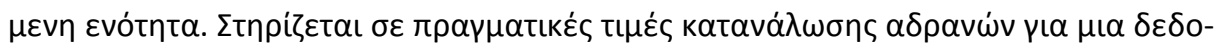

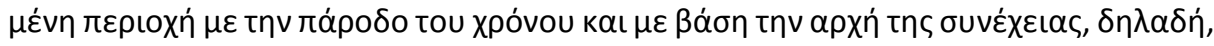

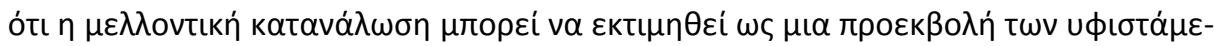

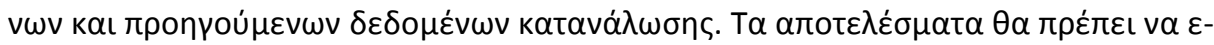

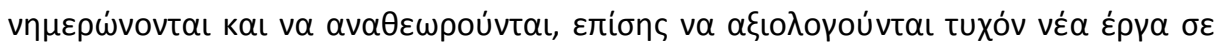

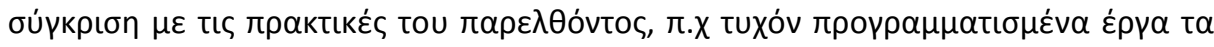

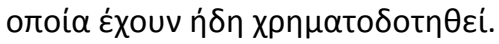

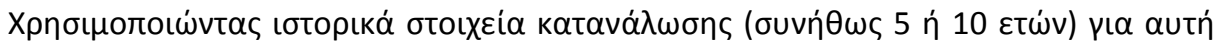

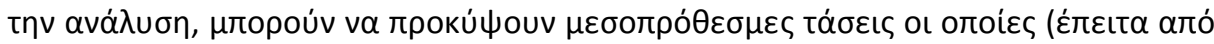

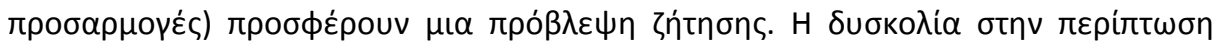

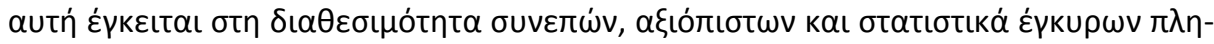

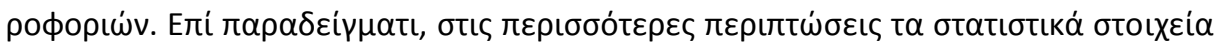

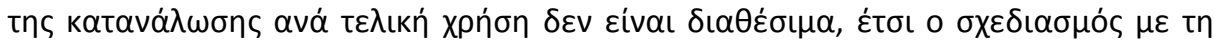

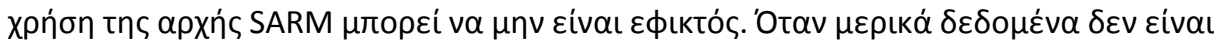

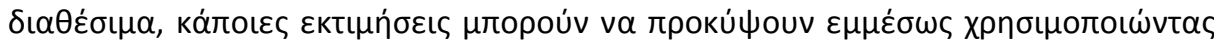

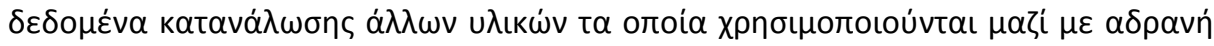

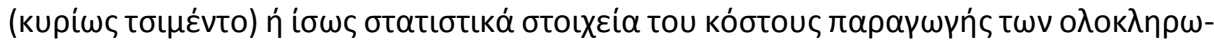

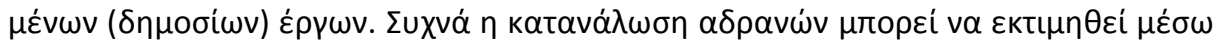

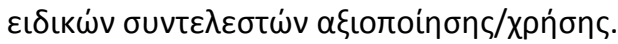

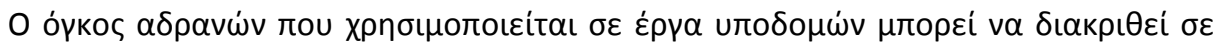

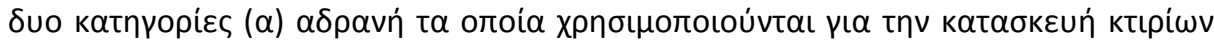

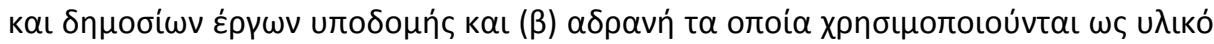




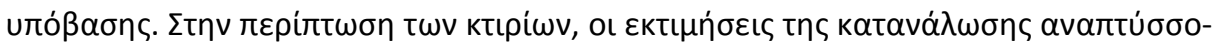

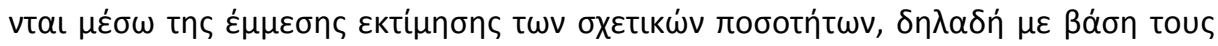

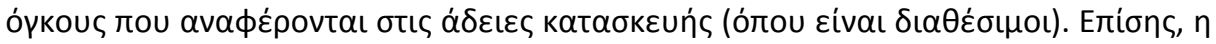

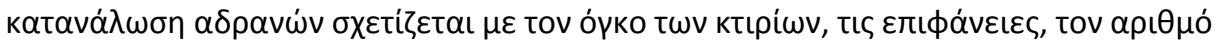

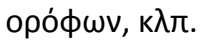

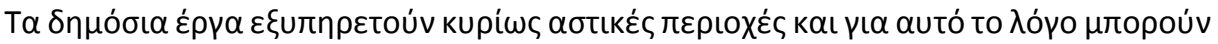

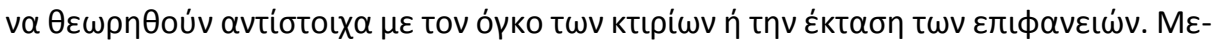

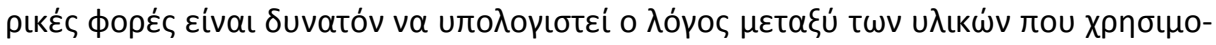

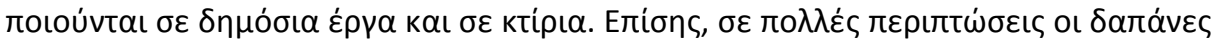

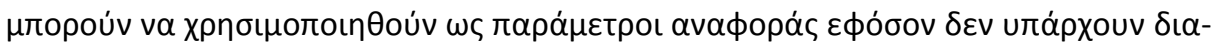

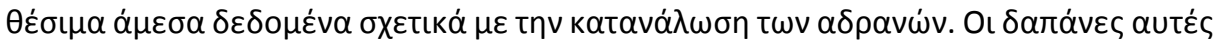

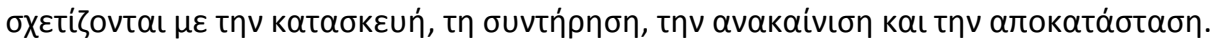

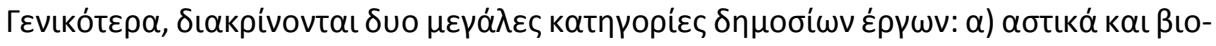

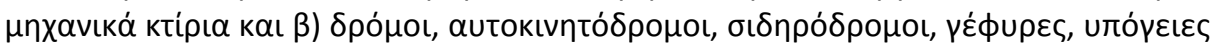

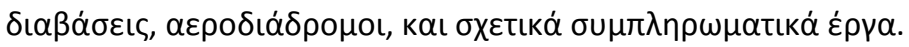

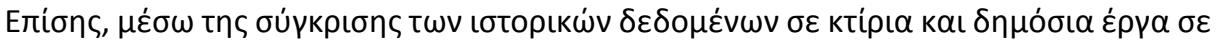

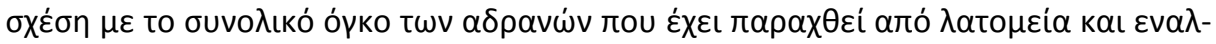

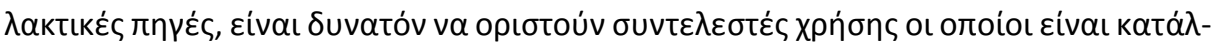

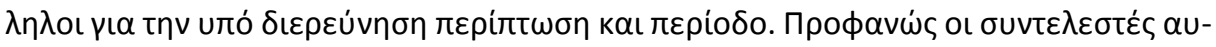

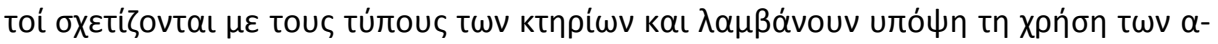

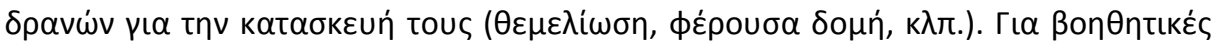

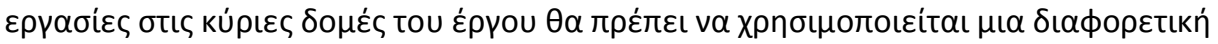

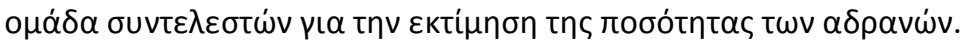

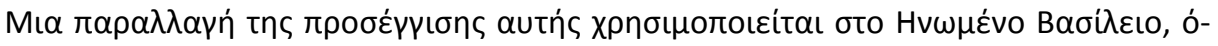

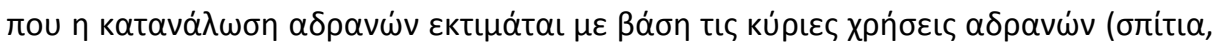

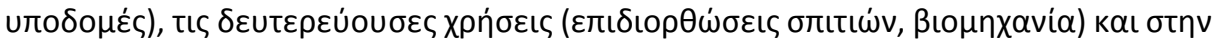

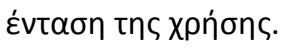

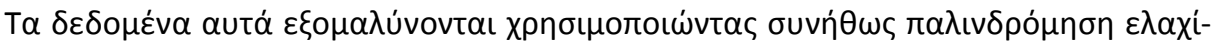

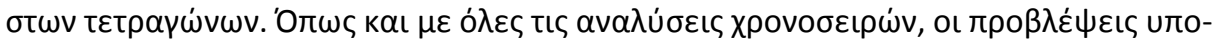
Ө

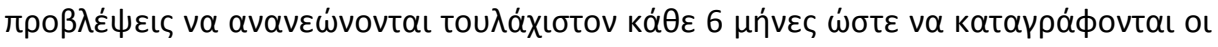
$\alpha \lambda \lambda \alpha \gamma \varepsilon \dot{~(U K ~ G o v, ~ 2014) . ~}$ 


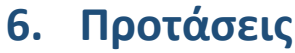

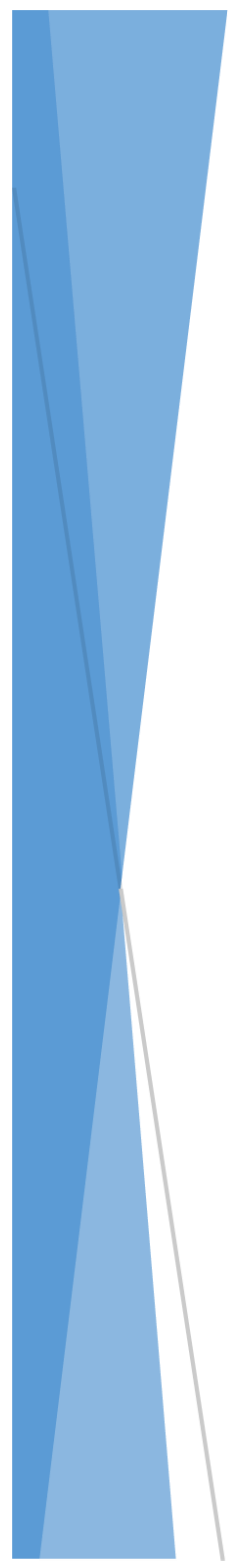

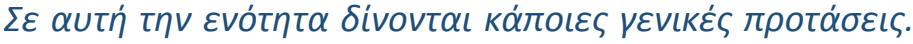

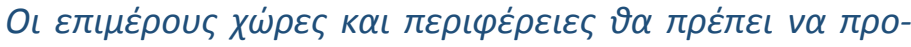

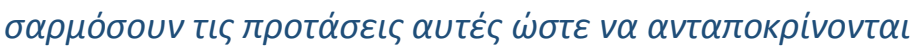

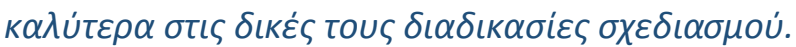




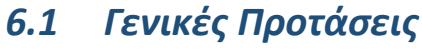

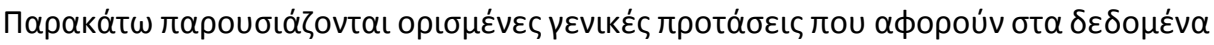

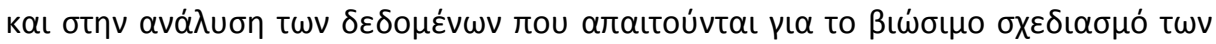
$\alpha \delta \rho \alpha v \omega \dot{v}$.

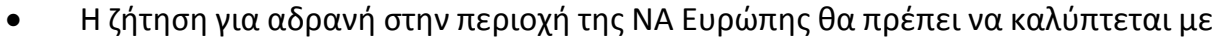

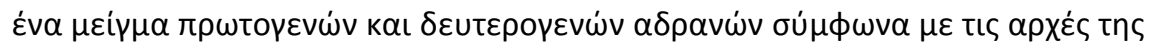

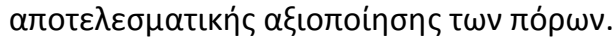

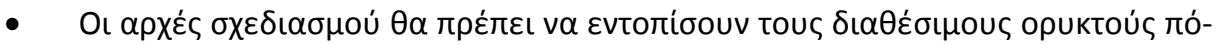

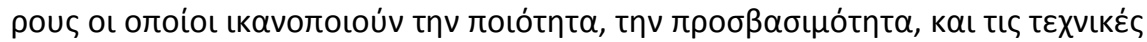

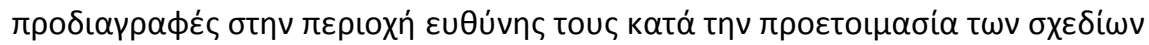

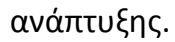

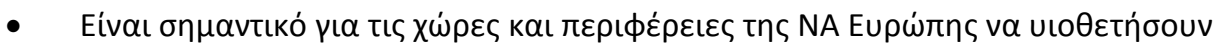

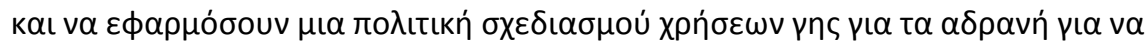

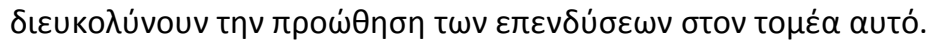

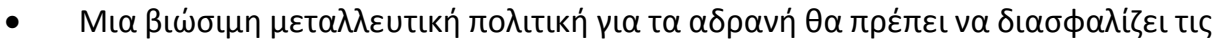

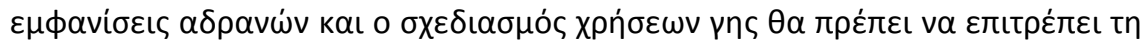

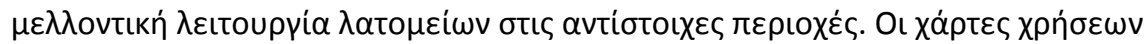

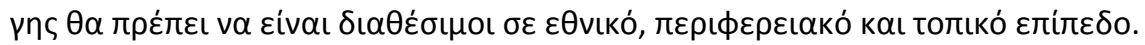

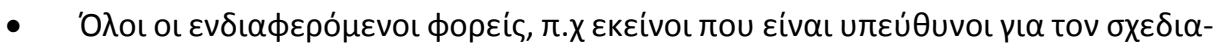

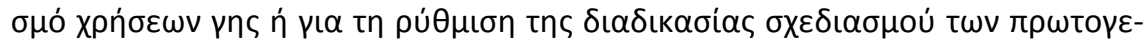

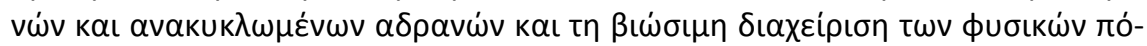

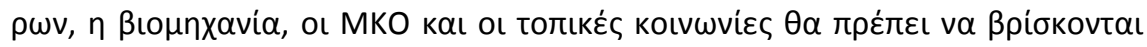

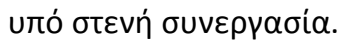

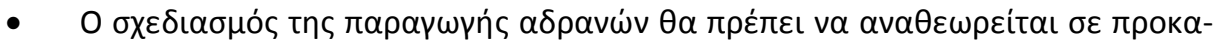

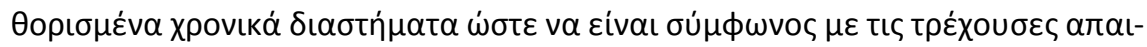

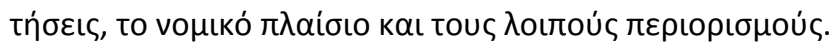

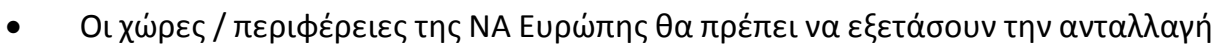

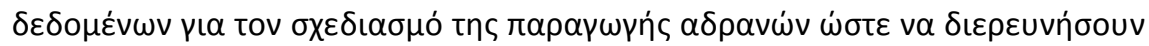

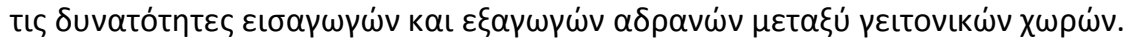




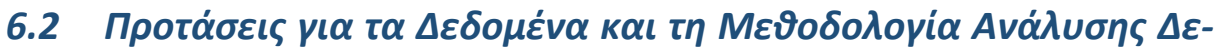

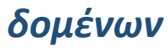

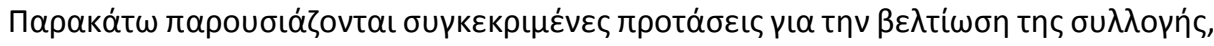

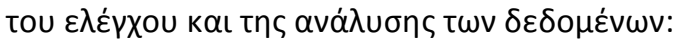

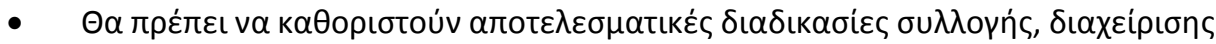

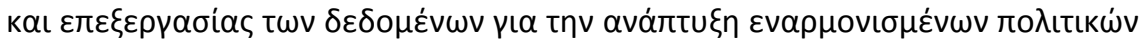

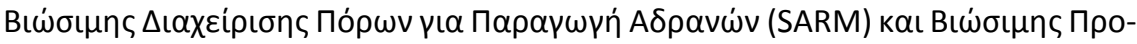

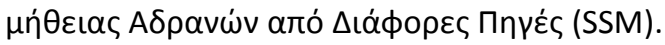

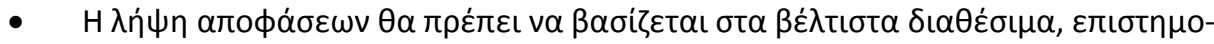

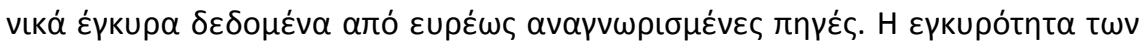

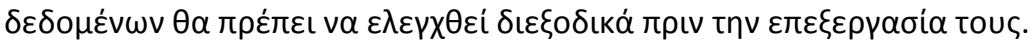

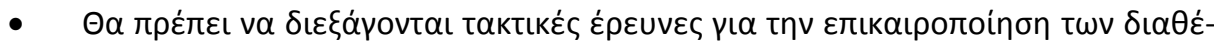

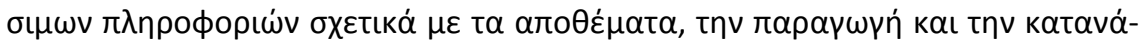

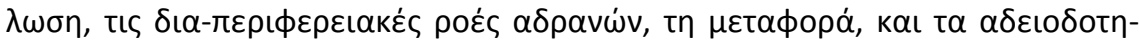

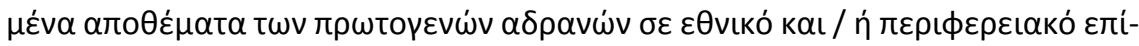

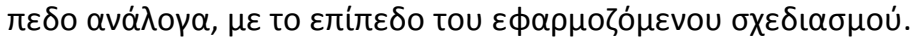

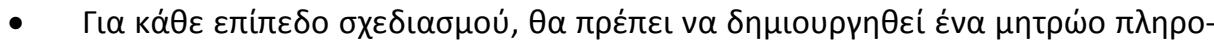

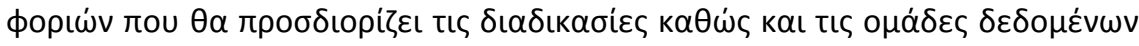

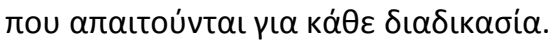

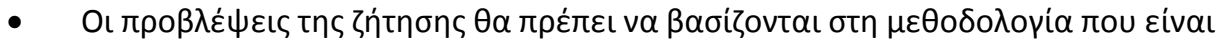

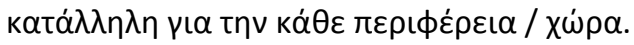

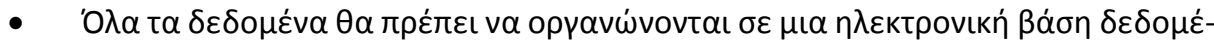

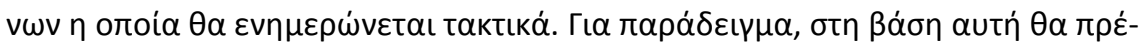

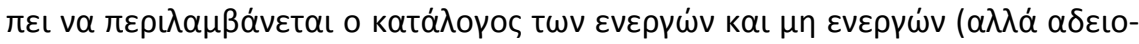

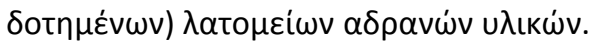

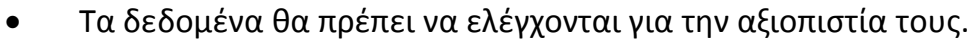


64 


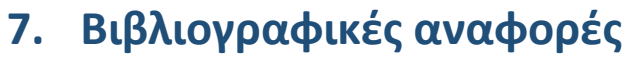

Agioutantis Z., Komnitsas, K. \& Athousaki, A. (2013). Aggregate Transport and Utilization in Urban Areas: Ecological Footprint and Environmental Impacts, Proceedings of the 13th International Congress, Chania, Greece, 5-8 Sept 2013, Bulletin of the Geological Society of Greece, vol. XLVII 2013.

Austria, (2014). http://www.en.bmwfw.gv.at/Energy/Seiten/TheAustrianMineralResourcesPlan.aspx

BGS (2014a). http://www.bgs.ac.uk/planning4minerals/Glossary.htm

BGS (2014b). http://www.bgs.ac.uk/planning4minerals/Resources_4.htm

BGS (2014c). http://www.bgs.ac.uk/planning4minerals/Resources_5.htm

BGS, (2012). "Aggregates safeguarding maps of Wales", Keyworth, Nottingham, British Geological Survey.

Blengini, G.A., Moltrer, A., Valbusa, M., Komnitsas, K., Agioutantis, Z. \& Garbarino, E. (2013). Data and methodologies for a resource-efficient planning of primary and secondary aggregates in South East Europe (SEE) countries, Proceedings, 6th International Conference on Sustainable Development Indicators in the Minerals Industry (SDIMI 2013), 30 June - 3 July 2013, Milos, Greece, pp. 282-288.

Blengini G.A., Bobba S., Shields D.J., Moltrer A. \& Valbusa M. (2014). Report on Data Analysis Methodologies, SNAP-SEE project.

Cabini, E., \& Zoboli, R. (2011). Ricerca per il dimensionamento dei volumi di sostanze minerali di cava per il nuovo Piano cave 2013-2023 della Provincia di Cremona. Alta Scuola per l'Ambiente - Università Cattolica di Brescia.

Chalkiopoulou, F. \& Hatzilazaridou, K. (2011). How to achieve aggregates resource efficiency in local communities, a joint manual, for stakeholders' decision making on the local level, SARMa Manual, http://www.sarmaproject.eu.

Hatzilazaridou, K. (2014). Data Dictionary, SNAP-SEE, http://www.snapsee.eu.

Leoben (2010). Planning Policies and Permitting Procedures to Ensure the Sustainable Supply of Aggregates in Europe, Final Report, Commissioned by UEPG, Department of Mineral Resources and Petroleum Engineering, University of Leoben, Austria, June.

Mazzanti, M., Paleari, S. \& Zoboli, R. (2007). Effectiveness of environmental taxes and charges for managing sand, gravel and rock extraction in selected EU countries Italy. 
SA, (2014). http://www.sustainableaggregates.com/sourcesof aggregates/recycle/

SARMa (2011). http://www.sarmaproject.eu/

SEA, (2001). Directive 2001/42/EC "on the assessment of effects of certain plans and programmes on the environment"

SPP, (2006). Scottish Planning Policy SPP4 "Planning for Minerals", Scottish Executive, www.scotland.gov.uk/publications/2006/08/30152427].

Springer (2014). http://www.springerreference.com/docs/html/chapterdbid/ 349568.html.

UEPG Annual Review 2012-2013. http://www.uepg.eu/uploads/Modules/ Publications/uepg-ar2012-2013_en_inter_v14_pbp_small.pdf

UK Gov (2014). https://www.gov.uk/government/uploads/system/uploads/ attachment_data/file/6286/forecastingaggregatesdemand.pdf

Welsh Assembly Government, Department for Communities and Local Government (2011) "Collation of the results of the 2009 aggregate minerals survey for England and Wales", Second Edition, October 2011.

Worchestershire County Council, (2013). Minerals Local Plan Background Document. Worcestershire Local Aggregates Assessment, http://www.worcestershire.gov.uk / mineralsbackground

http://www.aggregate.com/glossary-of-terms/\#sthash.oqjYPj46.dpuf http://www.businessdictionary.com/definition/demand-forecast.html http://economics.about.com/cs/economicsglossary/g/demand.htm http://www.oecd.org/environment/indicators-modelling-outlooks/MFA-Guide.pdf http://www.scotland.gov.uk/publications/2010/02/03132605/12 http://www.scotland.gov.uk/publications/2006/08/30152427/0 



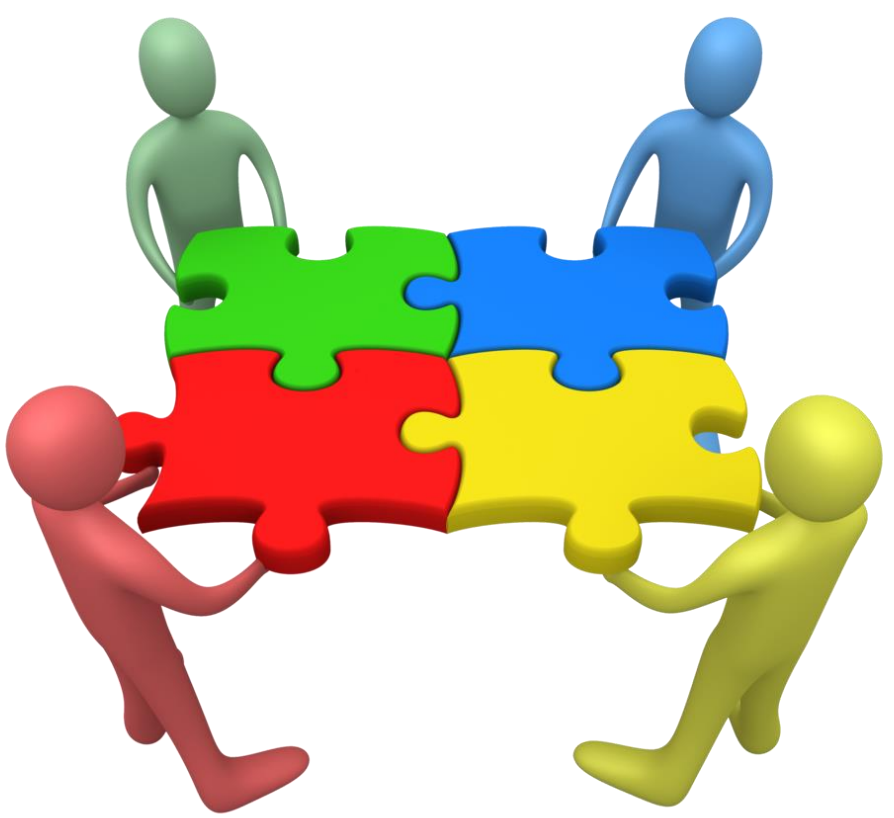

Sustainable Aggregates Planning in South East Europe (SNAP-SEE) 